
Geszler Nikolett Barbara

WORK-FAMILY CONFLICT OF HUNGARIAN MANAGER FATHERS 
Institute of Sociology and Social Policy

\author{
Tutor: \\ Dr. Nagy Beáta, CSc
}

(C) Geszler Nikolett Barbara, 2016 
Corvinus University of Budapest

Doctoral School of Sociology

\section{WORK-FAMILY CONFLICT OF HUNGARIAN MANAGER FATHERS}

Doctoral Thesis

Geszler Nikolett Barbara

Budapest, 2016 


\section{TABLE OF CONTENT}

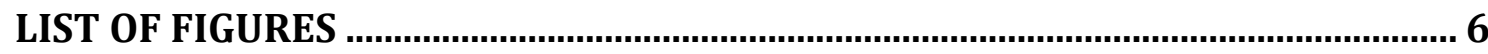

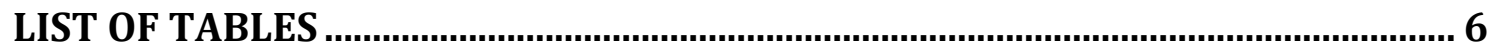

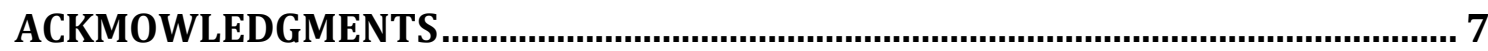

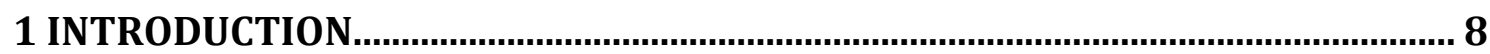

1.1 Defining the Problem ......................................................................................................

1.2 Research Questions, Aims and Relevance ………………………………………………....10

1.3 Structure of Study................................................................................................................11

2 THEORETICAL BACKGROUND...........................................................................12

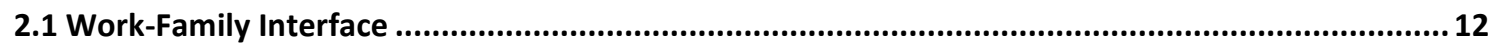

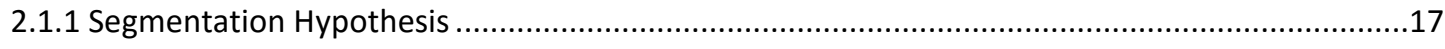

2.1.2 Compensation Hypothesis ............................................................................................

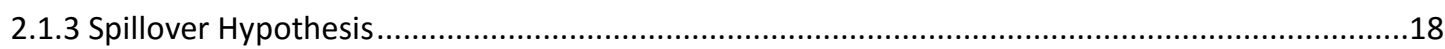

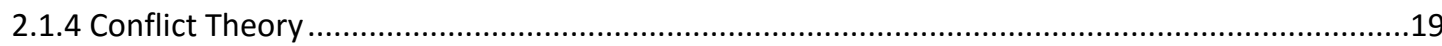

2.1.5 Positive Interactions............................................................................................................19

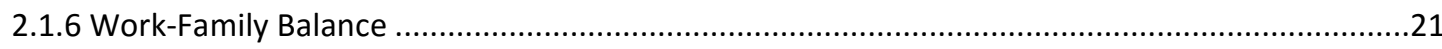

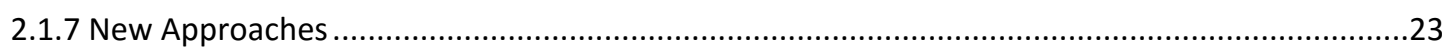

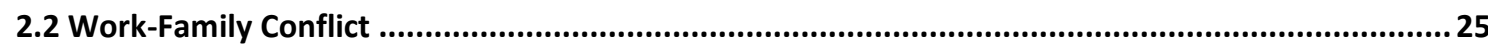

2.3 Time-Based Conflict and Post-Fordist Time Regime ....................................................................... 29

2.4 Behaviour-Based Conflict, Managerial Masculinity and Involved Fatherhood..............................35

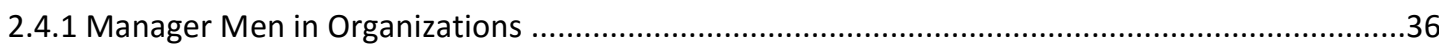

2.4 .2 Involved Fatherhood, Changing Masculinities .........................................................................39

2.5 Strain-Based Conflict, Job Stress and Social Support ..............................................................43

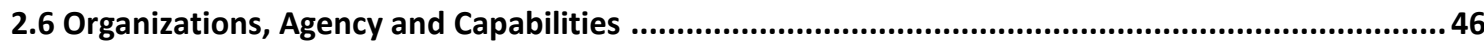

2.6.1 Organizational Contributions to Work-Family Balance.......................................................48

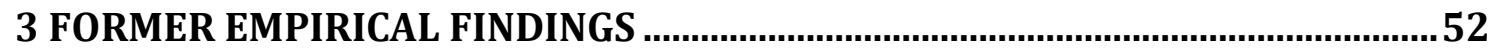

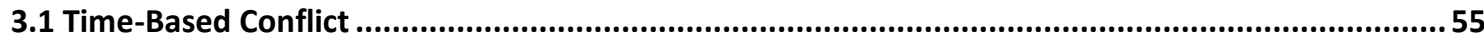

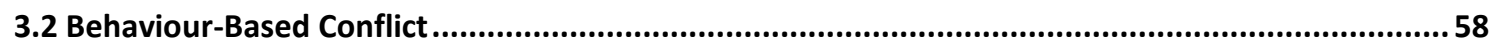

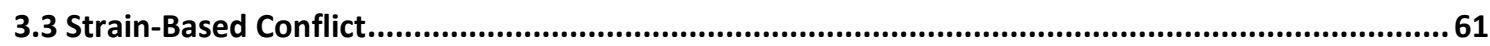

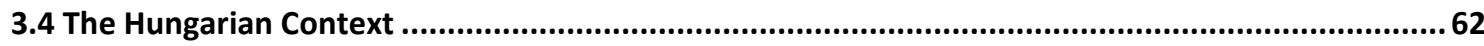

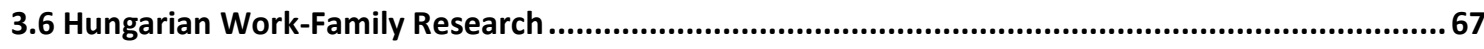

4 RESEARCH QUESTIONS, SAMPLE AND METHOD................................................... 74

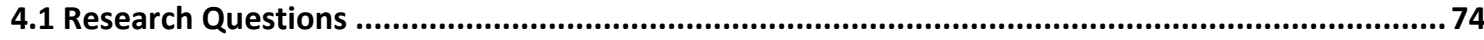

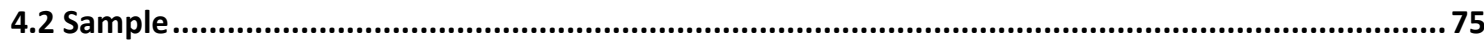

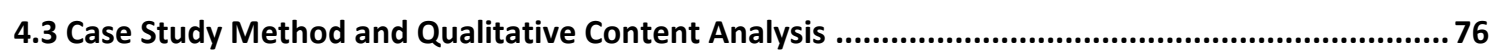

5 RESEARCH BACKGROUND .......................................................................................80 


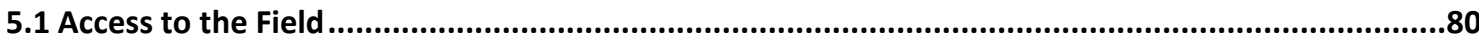

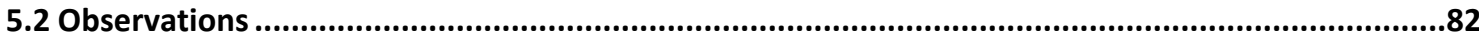

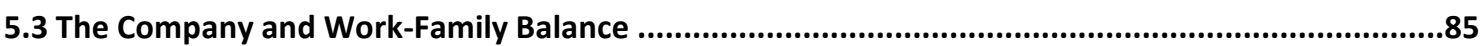

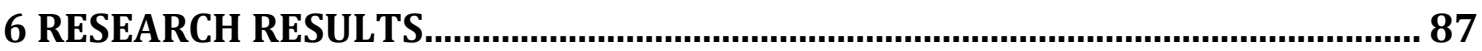

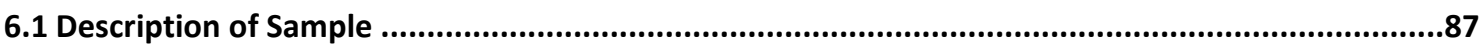

6.2 First Research Question: Work-Family Conflict...................................................................91

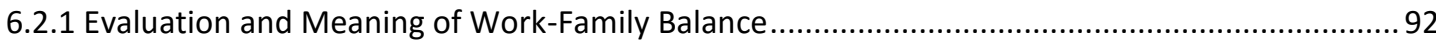

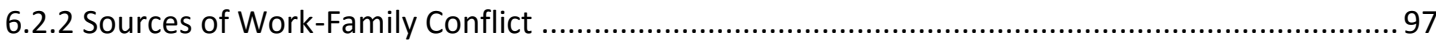

6.2.3 Consequences of Work-Family Conflict....................................................................... 103

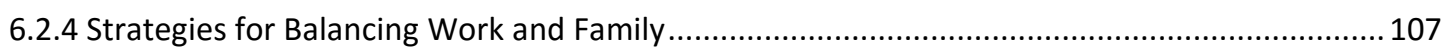

6.2.5 Female Managers and Work-Family Conflict .................................................................... 114

6.2.6 Discussion of the First Research Question ............................................................... 118

6.3 Second Research Question: Organizational support, Agency and Capabilities .............................123

6.3.1 Corporate Work-Life Balance Policy ..................................................................................... 123

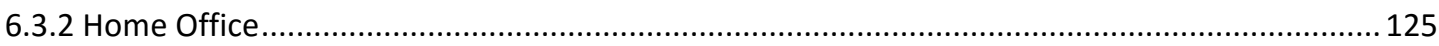

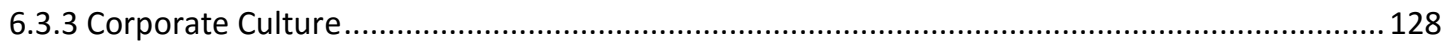

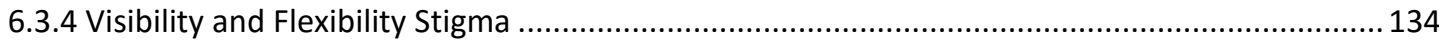

6.3.5 Agency and Responsibility in Work-Family Balance ........................................................ 138

6.3.6 Female Managers, their Agency and Capabilities................................................................ 141

6.3.7 Discussion of the Second Research Question................................................................... 144

6.4 Third Research Question: Managerial Masculinity and Involved Fatherhood ..............................149

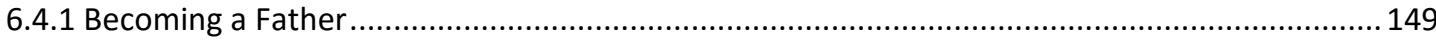

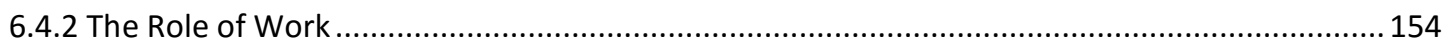

6.4.3 The Relationship between Management and Fatherhood .................................................... 156

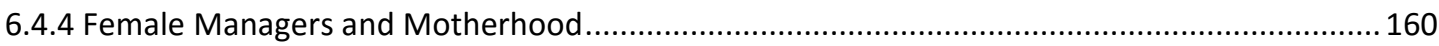

6.4.5 Discussion of the Third Research Question ............................................................... 162

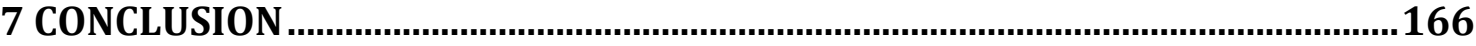

REFERENCES............................................................................................................... 174

OWN PUBLICATIONS REGARDING THE TOPIC ..................................................182

APPENDIX ........................................................................................................................ 182 


\section{LIST OF FIGURES}

Figure 1 Theoretical Foundation of the Study...................................29

Figure 2 Distribution of Interviews by Gender and Managerial Level.................87

Figure 3 Distribution of Interviews by Gender and Field of Work....................89

Figure 4 Distribution of Interviews by Level of Education and Gender...............90

Figure 5 Distribution of Interviews by Partners' Level of Education and Respondents'

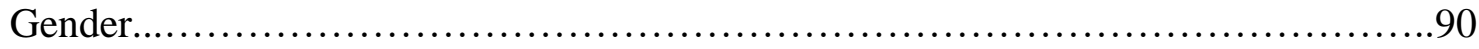

Figure 6 Distribution of Interviews by Partners' Type of Occupation and Respondents' Gender......

\section{LIST OF TABLES}

Table 1 Summary of Work-Family Interface Theories .16 


\section{ACKMOWLEDGMENTS}

First of all I would like to thank my tutor, Beáta Nagy, for her valuable remarks, professional guidance but most of all her support and encouragement when I felt uncertain. I could count on her advice anytime both in professional and personal way. I could not wish for a better supervisor.

I am grateful to György Lengyel who supported me from the beginning of my studies and involved me in researches that established my researcher path. I cannot thank him enough for these opportunities and that he trusted me.

I would like to thank my colleagues in the OTKA research group: Gábor Király, Zsuzsanna Géring, Ildikó Dén-Nagy and Veronika Paksi for the inspiring meetings, new ideas and friendly atmosphere. It was a pleasure to work with them.

I express my gratitude to Judit Takács, Henriett Primecz and Tamás Bartus for their precious advices, comments and questions which greatly contributed to the improvement of this work.

I am grateful to the influential professors and researchers I had the chance to meet in Oslo, Sigtona Halrynjo, Helene Aarseth and Anne Lise Ellingsæter. They had found time to review my work and give valuable remarks as well as inspire me with their great knowledge on Nordic gender issues.

I would like to thank my friend and group mate, Mounia Utzeri for her friendly and always cheering support. Moreover I am grateful to my best friend, Zita KovácsÖrdög, her ambitious plans and our long going co-operation motivated me to apply for the $\mathrm{PhD}$.

Last but not least I cannot be grateful enough for my dear parents, who have supported me in every possible way. Thank you! 


\section{INTRODUCTION}

\subsection{Defining the Problem}

Work-family balance, an issue which started to attract researchers' attention in the 1960s, is now one of the most popular research topics. Reasons for this include the significant social changes which have occurred primarily in Europe and the United States concerning gender, such as the increasing number of women in the labour force, the high rates of divorce which have resulted in many single-parent families, as well as the growing number of dual-earner couples and changing gender roles (Emslie - Hunt, 2009; OECD, 2008). In earlier studies, the focus was on examining educated, ambitious women who wanted to meet society's requirements of being both good mothers and wives and career women at the same time. The caring responsibilities imposed upon women affect their opportunities of holding down a full-time job, accumulating income, having equal opportunities and building up a successful career. Moreover, since women usually spend significantly more time on household chores and care work than their male partners, they are loaded with the 'double burden' of having to engage both in paid employment and in unpaid domestic labour (Eräranta - Moisander, 2011). The topic of work-family balance has gained importance from the state and an organizational viewpoint as well, since helping individuals to manage a career and a family can increase both birth rates and employment rates. The value of balancing work and family has become widespread in most European societies and embedded as a norm in EU documents and discourse on gender equality and family well-being (Hobson - Fahlén, 2009). From a business perspective, disharmony in one's life or dissatisfaction with work can cause deterioration in performance, accidents and higher levels of fluctuation and absenteeism. These effects may cause significant losses, a fact which increases the interest of companies in the issue of work-family balance (OECD, 2008; Nagy, 2008; Kelly et al., 2010).

Work-family balance, however, is still often regarded as an issue for women, even if the discourse about it is formulated in a gender-neutral way (Lewis, 2007). The reason for this is that the uninterrupted full-time working model of men is still often taken for granted (Burke, 2000). On the other hand, there are increasing expectations that men should take part in home and child care duties, especially in the Nordic countries, but in Western societies generally. Consequently, men who are required to 
face the difficulties of harmonizing work and life (just as working women do) are no longer rarities (Eräranta - Moisander, 2011; Halrynjo, 2009; Chesley, 2011; Duyvendak - Stavenuiter, 2004). Empirical findings confirm the proposition that men today are experiencing greater conflict with their work and family roles than ever before (Ladge et al., 2014). Especially after they become fathers, men too can find it difficult to 'have it all': that is, to have a satisfying job and the time to sustain good relationships in their communities, as well as with their children and partners (O'Brien et al, 2007). In men's lives, the risk is divorce and the loss of an important part of their social networks as a consequence of working long hours, neglecting familial responsibilities and being 'weekend fathers'. These factors create tension in their domestic relationships and also prevent them from gaining personally valuable experiences as fathers through more involved engagement and closer relationships with their children. For children, a family with a good work-family balance is an important component of a harmonious family environment (Eräranta - Moisander, 2011). In terms of gender equality, Hungary is traditional compared to Nordic and Western-European countries. Fathers who share paid and unpaid labour with their partners according to traditional gender norms tend to face less conflict between their work and family demands (Van der Lippe et al., 2006). This does not mean, however, that work-family problems do not concern Hungarian fathers. Due to the slow changes concerning the duties of fatherhood, Hungarian fathers may face dual expectations: they are required to correspond to the role of being the traditional male breadwinner model on the one hand, but demonstrate emotional and care-related commitment towards their children at the same time (Spéder, 2011).

Harmonizing work and family in the lives of fathers can be even more difficult if they have demanding, time and energy-consuming jobs; for example, if they occupy managerial positions. Empirical results show that men in managerial positions are often subject to a high level of conflict (Allard et al, 2007). Competition-driven pressure to be responsive and flexible has forced organizations to downsize and reduce hierarchical levels, adding complexity to managers' jobs (Mayo et al., 2011). Therefore managers not only face longer working hours, but a high level of responsibility (Szalma, 2014). In addition, leaders are important in times of organizational change, especially in new fields and types of change such as promoting the work-family balance in companies. Managers are in position to help or hinder the development of a family-supportive organizational culture, and they can be role models for other employees (Allard et al., 2007; Holter, 2007). Still, there is currently a lack of studies that focus on managerial 
men and the work-family balance in general (Mayo et al., 2011), particularly in Central and Eastern European countries.

\subsection{Research Questions, Aims and Relevance}

The purpose of this thesis is to analyse the conflicts between work commitments and the family life of Hungarian fathers in managerial positions. The research questions facilitate analysis of how manager fathers experience conflicts between their work and family life, how the organizations they are working for influence their ability to achieve work-family balance, especially in terms of flexibility, and, finally, how discourses about ideal employees or involved fathers affect their work-family balance. To answer these questions a case study was undertaken by the Hungarian subsidiary of a Scandinavian multinational service sector company, wherein 43 personal interviews were made with manager fathers and an additional 15 interviews with manager mothers as a control group. The Scandinavian origin of the company is important in the sense that Nordic societies are well-known for their longstanding policy legacy of promoting gender equality and work-family balance in the workplace (O'Brien et al, 2007). Therefore, this aspect of organizational influence on managers' work-family balance was taken into account. The interviews were analysed according to theory-based method of structuring qualitative content analysis, using NVivo10 software.

The aim of this study is thus to enrich the Hungarian literature about the workfamily interface and to fill the research gap about men. The novelty of this research is that it combines different aspects of work-family conflict, covering time-, strain- and behaviour-related problems with a focus on the special sample of manager fathers. The aim is to explore a broad scope of work-family conflict mechanisms through this case. In addition, it is designed to merge the concept of work-family conflict with masculinities studies and management literature by shedding light on the complex interrelations that exist between managerial position, fatherhood and work-family interactions. For this study of the organizational context individual work-family behaviours and acts are embedded in, it is indispensable to understand why certain work-family balance strategies work, while others fail. This work draws attention to the role of the company in employees' work-family balance and thus may be a useful source of information to managers, both as employees trying to harmonize their own work and family lives, and as leaders through their influence on corporate culture. 


\subsection{Structure of Study}

The dissertation will start with a literature review, where the main theories and concepts regarding the interface between work and family will first be presented. This summary has a specific focus on work-family conflicts, since this approach is the frame of the literature review. Along the three conflict types (time-based, behaviour-based and strain-based conflict) the dissertation outlines other relevant concepts regarding masculinity, fatherhood, work-family balance and organizations. Connected to timebased conflict, the globalised, knowledge-based, highly committed working environment described by the term 'post-Fordist time regime' will be presented. Regarding behaviour-based conflict, the discrepancy between the ideal manager and involved father is outlined as a potential source of work-family conflict. Here, some of the characteristics of managerial masculine identity will be described on the one hand, and involved fatherhood on the other hand. In relation to strain-based conflict some important psychological and physical consequences of stress will be summarized in general. Then the focus is narrowed to job stress, social support and how perceived control over boundaries between work and family can affect the psychological wellbeing of individuals. In the next chapter, organizational work-family and familyfriendly initiatives and their effect on employees' agency and capability to claim for work-family balance are described. This is followed by a brief overview of international work-family conflict findings, and finally the Hungarian context and empirical results about work-family questions close this chapter.

After the literature review the research questions are formulated based on the theoretical concepts and empirical findings previously presented. This material is followed by a summary of the applied case study method and qualitative content analysis method, as well as details of the sampling design. The description of research starts with an overview of the data collection which includes a list of difficulties, experiences and observations regarding access to the company and the interview situations. This is followed by a demographic summary of the sample and the presentation of interview results. The research findings are divided into three main chapters according to the three research questions. The summary of these three parts ends with a discussion sub-chapter, and the dissertation is then closed with the conclusion. 


\section{THEORETICAL BACKGROUND}

\subsection{Work-Family Interface}

A search for the term 'work-family interface' among Jstor journal articles (limited only to the last 10 years) lead to 240182 search results. This high number demonstrates well the popularity of work-family research. The first literature regarding the interaction between work and family appeared in the 1950s and research into the topic had become widespread by the end of the 1960s (Grzywacz - Marks, 2000; Gregory - Milner, 2009). Initially, the terms work and family were used to describe the relation of the two life spheres. Work and home were seen as separate domains as the result of the industrialization of the 19th century, where home was the feminine private sphere, and work the masculine public domain (Korabik et al., 2008). The family model of industrialization developed along this division of paid and unpaid labour, with the man as breadwinner and woman as caregiver (Tammelin, 2009). Due to the constant increase in participation of women in the labour market and their intensifying financial independence, the number of single parent households and dual-earner families has grown (Emslie - Hunt, 2009; OECD, 2008). The spread of the dual-earner family model was further enhanced by the strengthening of the service sector that provided women with more opportunities than in the male-dominated industrial sector, as well as created new norms about ideal living standards through intensifying consumption. To maintain this living standard in a society based on consumption, one earner in the family was not always sufficient (Korabik et al., 2008). Along with the increase of women in the labour force, the participation of men in unpaid labour developed more slowly (Maume et al., 2010). The gender gap regarding participation in unpaid labour has decreased since the 1960s; this can be explained by the fact however that women (on average) have reduced time spent on chores (Korabik et al., 2008; Emslie - Hunt, 2009; Maume et al., 2010). Thus the traditional gender specialization of domestic tasks has remained remarkably resilient (Wajcman - Edwards, 2005): it is still women who shoulder the burden of the bulk of household and caring duties. Consequently, they are responsible for 'the second shift'; namely, for performing domestic labour in addition to doing paid work (Hochschild - Maschung, 1989). These transformations of family forms, the division of labour and gender roles make relevant the analysis of how women harmonize the dual 
demands of work and family life, since how they manage work and family spheres have major implications for well-being, job performance and family functioning (Frone, 2003).

The shift from industrial-based economies towards service and informationbased economies made life more complex, not only for working mothers but for men as well and for individuals without family obligations (Hill et al., 2004). By focusing only on employees with family responsibilities, the notion of work-family balance was considered in practice as liable to trigger a backlash in the workplace from non-parents (Gregory - Milner, 2009), As a result, since the 1990s the expression 'work-life' has emerged. The introduction of 'life' to the term brings a conceptualization that is more gender-neutral and inclusive, covering the needs of both men and women, and those without family responsibilities. Thus the linguistic shift reflects a broader way of framing the topic to engage men and women with and without children or other caring commitments (Lewis, et al., 2007).

As can be seen, some part of 'life' involves obligations such as housework or care tasks which must be undertaken even if one does not feel in the mood. The other dimensions of 'life' are, however, voluntary activities that make people fulfilled, such as spending time with children, partners, friends, being part of a community, or having hobbies. In addition, the line between these obligations and leisure-time activities is not always clear. For example, cooking might be a time-consuming duty for one person, while for another it might be a passion. Even for the same person an activity might be an obligation in one situation and fun in another - for example, taking children to the playground (Primecz et. al, 2014). Thus both the concept of 'work-family' and 'worklife' is used in practice, research and policy debates, although the appearance of the former, narrower category is more frequent. (Tammelin, 2009; Rantanen, 2008). Even the concept of 'work' - usually associated with paid work - is not completely unequivocal if we take into consideration unpaid overtime, commuting time or technological innovations which make working from home possible (Guest, 2001). This dissertation focuses primarily on work and family domains, although the term 'worklife' is also used, especially for describing the research findings.

The relationship of work and family can be described by the term work-family interface, which includes every practice and experience of how life spheres come together (Tammelin, 2009). The work-family interface can be further characterized through four components: amount, direction, context and quality (Rantenen, 2008). 
Amount refers to the degree of segmentation or integration between the two domains. In terms of segmentation, there are strict physical, temporal, functional and psychological boundaries between work and family life which are thus seen as relatively independent and non-influential spheres in relation to each other. By contrast, integration can be described as tight interdependency between the two domains in terms of time, place, people, behaviour, thoughts and emotions. It is important to note that there is a reasonably strong consensus in the literature that work and family should be regarded as two domains in strong interaction, not as separate and independent spheres (Grzywacz - Marks, 2000; Emslie - Hunt, 2009).

Direction determines the reciprocal relationship between work and family domains and indicates its direction. Work can affect family life (work-to-family direction), but the opposite is possible too: family can affect working life (family-towork direction), or the two effects may appear simultaneously. Nowadays it is also generally accepted within the literature that the work-family interface is a bi-directional and double-layered phenomenon where work can interfere with family and family with work (Greenhaus - Beutell, 1985).

Context means the perspective from which the work-family interface is studied. Personal relations and individual experiences both in work and family domains reflect the microsystems of the work-family interaction (Voydanoff, 2002). The linkages and processes that occur between an individual and the other role partners create the mesosystem of the work-family interface. The exosystem includes those influences which come from a third life domain, in which an individual is not directly involved but is still affected. Such an exosystem can be the working environment of one's partner. Finally, the macrosystem is constituted by the broader social contexts, such as subcultures or larger groups with distinctive norms, culture, attitude and rules (Tammelin, 2009). Therefore preferences are not only formed by individual values and predispositions, but by broader social factors too, like the quality of the system of childcare, or the condition of the labour market (Gregory - Milner, 2009). Most of the literature has investigated the individual, micro level, putting great emphasis on psychological aspects, while fewer pieces of research have investigated the influence of broader social systems, or the effect of the exosystem on the individual's work-family interface (Grzywacz - Marks, 2000).

The quality dimension refers to negative versus positive interactions between work and family spheres. The negative work-family interface includes the concepts of 
work-family conflict, negative spillover and resource drain. These approaches are rooted in role stress theory and scarcity concept (Greenhaus - Beutell, 1985). To positive work-family interaction such concepts as work-family enhancement, enrichment, positive spillover and facilitation belong. The basis of these concepts is accumulation and expansion theory (Sieber, 1974). Thus work and family can influence each other both in negative and positive ways, but this does not necessary mean that these are the two endpoints of the same continuum. Since some approaches claim that negative and positive influences can appear independently, an individual might experience both of them at the same time. The literature is, however, dominated by the negative concepts - the positive effects have started being acknowledged only since the 1970 s, and research in this area is not yet as well established as that which exists for the negative approaches (Grzywacz - Marks, 2000; Voydanoff, 2002).

Four specific theoretical models have been used extensively in the study of individual experiences of work-family interface: segmentation hypothesis, compensation hypothesis, spillover hypothesis and conflict theory (Tammelin, 2009). These are followed by the positive approaches to and new models of the work-family interface, like border theory or ecological system theory. All these approaches are middle-range theories that originate primarily from psychology and have their focus on the individual level, and build on role theories as their primary foundation. Structuralfunctionalist role-theory assumptions are based on the gendered separation of work and family spheres on biologically-based proclivities (Grzywacz - Marks, 2000). The second wave of studies on men and masculinities strongly criticized the idea of functionally separated gender roles, since they lack the elements of power inequalities and dynamics, as well as relations between the individual acts and social structure (Hadas, 2011). The work-family interface approaches, however, rarely place the emphasis on the gender aspect of social role theory. Instead, they distinguish roles according to domains, such as work roles (manager, employee, union representative), family roles (spouse, parent, offspring), religious roles, community roles, student roles or leisure roles (Frone, 2003). Sociological work-family research also applies these initially psychological models to describe the relationship between work and family domains, and as a result, the introduction of these terms is inevitable. In the following Table the main attributes of these basic work-family approaches will be briefly summarized with a specific focus on conflict theory, which is the main theoretical foundation of this dissertation. 


\begin{tabular}{|c|c|c|c|}
\hline INTERFACE & ASSUMPTION & AMOUNT & QUALITY $^{1}$ \\
\hline segmentation & $\begin{array}{l}\text { "posits no systematic relationship between work and } \\
\text { non-work roles" (Rothbard - Dumas, 2004:74) }\end{array}$ & segmentation & \\
\hline compensation & $\begin{array}{l}\text { "people compensate for dissatisfaction in one domain } \\
\text { by trying to find more satisfaction in the other domain" } \\
\text { (Roehling et al., 2003:102) }\end{array}$ & segmentation & $\begin{array}{l}\text { positive/ } \\
\text { negative }\end{array}$ \\
\hline spillover & $\begin{array}{l}\text { "the transfer of mood, affect, and behaviour between } \\
\text { work and home" (Roehling et al., 2003:101) }\end{array}$ & integration & $\begin{array}{l}\text { positive/ } \\
\text { negative }\end{array}$ \\
\hline conflict & $\begin{array}{l}\text { "a form of inter-role conflict in which the role pressures } \\
\text { from the work and family domains are mutually } \\
\text { incompatible in some respect." (Greenhaus - Beutell, } \\
\text { 1986:77) }\end{array}$ & integration & negative \\
\hline $\begin{array}{l}\text { positive } \\
\text { interface }\end{array}$ & $\begin{array}{l}\text { enhancement: "the extent to which aspects of the work } \\
\text { or family role provide resources that facilitate the } \\
\text { performance of the other role" (Voydanoff, 2002:149) } \\
\text { enrichment: "the extent to which experiences in one } \\
\text { role improve the quality of life in the other role" } \\
\text { (Greenhaus - Powell, 2006: 73) } \\
\text { facilitation: "represents the extent to which } \\
\text { participation at work (or home) is made easier by virtue } \\
\text { of the experiences, skills, and opportunities gained or } \\
\text { developed at home (or work)" (Frone, 2003: 145) } \\
\text { positive spillover: "occurs when satisfaction } \\
\text { and stimulation at work translate into high levels of } \\
\text { energy and satisfaction at home" (Roehling et al., } \\
2003: 101 \text { ) }\end{array}$ & integration & positive \\
\hline balance & $\begin{array}{l}\text { "satisfaction and good functioning at work and home, } \\
\text { with a minimum of role conflict" (Clark, 2000:751) }\end{array}$ & integration & neutral \\
\hline $\begin{array}{l}\text { ecological } \\
\text { theory }\end{array}$ & $\begin{array}{l}\text { "work-family experience is a joint function of process, } \\
\text { person, context and time characteristics" (Grzywacz - } \\
\text { Marks, 2000:112). }\end{array}$ & integration & neutral \\
\hline border theory & $\begin{array}{l}\text { "explains how individuals manage and negotiate the } \\
\text { work and family spheres and the borders between them } \\
\text { in order to attain balance" (Clark, 2000:750) }\end{array}$ & $\begin{array}{l}\text { segmentation } \\
/ \\
\text { integration }\end{array}$ & neutral \\
\hline
\end{tabular}

Source: Author's construction

\footnotetext{
${ }^{1}$ Context is not included here since the type of interface does not determine whether the research focuses solely on the individual level or includes other levels as well. Direction is not indicated either, because there is a consensus in the literature about the bi-directional nature of all work-family interface models.
} 


\subsubsection{Segmentation Hypothesis}

The segmentation hypothesis postulates that no systematic relationship exists between work and family. According to this claim, work and family can be understood as two separate domains which do not affect one another (Rothbard - Dumas, 2004). Such segregation reflects the demographic and attitudinal realities prevalent in the 1960s and 1970s when work and home, and the public and private were treated as separate spheres which were characterized with clear gender segregation. Initially, segregation was seen as the natural division of the two domains because of their separation in time, space and function (Dubin, 1973). The concept was used first of all to describe the situation of women since it was mainly them who had to face the conflicting demands of the two domains. Besides the case of women, the segregation perspective has also been applied in research into blue-collars workers who have more unsatisfying jobs, and for whom the segmentation of the two domains is seen as a natural process. This view is reinforced by prevalent corporate culture that requires that employees do not allow family matters to interfere with work responsibilities (Martinengo, 2007). However, research suggests that segregation does not occur naturally but is the result of conscious effort; an active psychological process of maintaining a boundary between work and family. Consequently, the more recent view is that work and family are closely related domains (Rothbard - Dumas, 2004).

\subsubsection{Compensation Hypothesis}

According to compensation hypothesis, what the individual lacks in the life domain can be complemented by the other life sphere (Guest, 2001). Thus, for example, employees may compensate or make up for a lack of satisfaction or deprivation experienced at work by trying to find more satisfaction and positive experiences in their family lives. Of course, the opposite is also possible, when work becomes the source of compensation. Individuals can compensate for their dissatisfaction in one role in different ways. First, an individual may reduce their involvement in one domain that is producing dissatisfaction while increasing their involvement in a more satisfying domain. For example, a worker may spend more time and become more engaged with work if experiencing negative affect from the family. Second, an individual may pursue rewards in one domain as a response to dissatisfaction in another domain - for instance, temporarily seeking fulfilment in family life when facing disappointment at work 
(Rothbard - Dumas, 2004; Champoux, 1978). This form of compensation may further be differentiated into so-called supplemental and reactive compensation. Supplemental compensation is sought out when rewards in one domain are insufficient and an individual seeks to obtain supplementary reward in the compensatory domain. Reactive compensation, on the other hand, occurs when a person seeks contrasting experiences in one domain to compensate for negative experiences in the other. Thus in both forms of compensation, reward is the focus, but while the supplemental compensation is sought out due to a lack of insufficient positive experience (such as when people seek autonomy at home to compensate for a lack of it at work), reactive compensation is the consequence of too many negative experiences (such as when a worker rests at home after a difficult day at work) (Martinengo, 2007). Similarly to the segregation hypothesis, compensation also assumes that work and family spheres are isolated constructs. Accordingly, it has been used less frequently in the literature (Tammelin, 2009).

\subsubsection{Spillover Hypothesis}

Spillover hypothesis postulates that positive or negative moods, emotions, attitudes, skills and behaviour experienced in one domain transfer over to other life spheres, generating similarities between the domains (Roehling et al., 2003; Rothbard Dumas, 2004). The concept explains that a person is not 'segmented' into a worker and a parent, for example, but each role coexists at the same time (Tammelin, 2009). Moreover, it no longer considers work and home to be separate domains but offers a casual model, positing that what happens in one domain can have a casual impact on what happens in another life sphere (Frone, 2003). Spillover can take two forms: it may happen because of the similarity between constructs in the two domains, for example, when someone who is highly satisfied with his or her work becomes highly satisfied with his or her experiences at home. The second form occurs when experiences are transferred from one domain to the other, such as when work stress affects negatively the atmosphere in the family, or when work-related fatigue is displayed at home (Rothbard - Dumas, 2004). It can be seen that spillover can be either positive or negative. The spillover model is the model most frequently utilised to describe the experiences of working people (Roehling et al., 2003). Negative spillover and conflict theory are very similar concepts, since both examine the transfer of emotions and behaviours between work and home. Rantanen (2008) explains the difference by stating 
that negative spillover focuses on the similarities between work and family as the result of the transfer of negative feelings, behaviour and moods, while conflict refers to the incompatibilities between roles. Roehling and co-authors (2003) add that conflict research also assesses how work-related tasks intrude on the family atmosphere, and vice versa.

\subsubsection{Conflict Theory}

Conflict theory has been the most widely used concept in work-family studies since the 1980s (Grzywacz.-Marks 2000). The role of conflict theory is based implicitly on the scarcity perspective (or resource drain model (Frone, 2003)), which assumes that time and energy as scarce resources can be easily depleted when individuals participate in multiple roles and the obligations of different, separate domains compete for a fixed and limited quantity of these (time, physical energy and psychological) resources (Grzywacz.-Marks 2000). As a consequence, work-family conflicts can be regarded as inter-role conflicts where the demands of work and family roles are incompatible and participation in one role makes it difficult to be involved in other roles (Greenhaus Beutell, 1985). Work-family conflict is a two-dimensional construct, where work can interfere with family, and family with work; both directions have different antecedents and can result in a variety of negative consequences (Grzywacz.-Marks 2000; Greenhaus - Beutell, 1985; Frone, 2003). Considering that this concept is the core of this dissertation, it is discussed in detail in the next chapter.

\subsubsection{Positive Interactions}

Theories that emphasize the positive aspects of work and family first appeared in the 1970s (Tammelin, 2009). These concepts are based on role accumulation theory and expansion approach. The basic argument for both is that having multiple roles in everyday life is not harmful, but rather beneficial to individuals. According to Sieber (1974), role accumulation theory explains (using four reasons) why holding multiple roles is advantageous: first, each role has its inherent and emergent privileges and advantages which benefit the individual in their own way. Therefore, the more roles an individual occupies, the more privileges can be enjoyed (role privilege). Second, multiple roles support overall status security since an individual's well-being is not dependent only on a single role (status security). Third, interacting with different role partners provides a variety of resources through social relationships which results in 
status enhancement and role performance (status enhancement). Finally, role accumulation includes the potential for personality enrichment and the emergence of an individual's personal desires and preferences through becoming involved with various role partners, increasing flexibility and tolerance and obtaining many sources of information (personality enrichment). Consequently, work can benefit family life, for example, via increased personal well-being, while family can benefit work, for instance, via stress management and spousal support (Grzywacz.-Marks 2000). Thus multiple role participation may lead to energy expansion, gratification and greater self-esteem (Martinengo, 2007). The expansion approach - in contrast to the scarcity concept assumes that human time, energy and commitment are not finite resources, since valuable and rewarding social activities and interactions have the potential to reproduce these resources thereby compensating for shortages of time. Based on this argument, role conflict is not the inevitable consequence of multiple roles since such roles offer the same potential for role expansion and resource enrichment (Rantanen, 2008).

The literature on positive interactions uses many similar definitions to describe the relationship that exists between work and family. One term is positive spillover, which was previously described. Another widespread concept is the work-family enhancement approach. This hypothesis can be associated with the idea of positive spillover, assuming that involvement in multiple roles does not necessarily mean a reduction in energy (Roehling et al., 2003). Of course, role accumulation not only depends on the number of roles, but also on the nature and social characteristics of these roles (Martinengo, 2008). According to Voydanoff (2002:149), enhancement is "the extent to which aspects of the work or family role provide resources that facilitate the performance of the other role".

Another very similar term, facilitation, refers to the extent to which experiences, skills and opportunities which are obtained as a result of active participation in one role improve the quality of involvement in the other role (Grzywacz - Marks, 2000; Rantanen, 2008). Both facilitation and enhancement are based on the extent to which aspects of the work or family supply resources that assist the other life sphere (Voydanoff, 2002).

A fourth definition that describes positive interaction between work and family is enrichment. According to the definition supplied by Greenhaus and Powell (2006), enrichment occurs when experiences in one role improve the quality of life in the other 
role. For example, when work successes improve one's general satisfaction and mood at home as well.

These four definitions are similar in the sense that they all assume a bidirectional positive effect between work and home. According to Greenhaus and Powell (2006), the enrichment concept is the most comprehensive approach among the positive interactions and can be used as an umbrella concept for all positive interactions. There is a slight difference in the outcome of these interfaces, however: while in enhancement and facilitation the positive effects of interface emerge as better engagement and performance in one life domain, in the case of enrichment and spillover it is not personal traits which improve, but the recipient domain's quality of life (Rantanen, 2008).

\subsubsection{Work-Family Balance}

Work-family balance is the most well-known term, not only in the literature but also in public discourse, indicating that balance is important for an individual's psychological well-being. Overall satisfaction and a general sense of harmony can be seen as indicators of a successful balance between work and family life (Clark, 2000; Greenhaus et al., 2003). Opinions differ, however, about how we should understand, define and measure work-family balance (Grzywacz - Carlson, 2007). Moreover, as Greenhaus and co-authors (2003) emphasize, many reviews of work-family relations either do not mention the work-family balance, or they mention balance but do not explicitly define the concept, nor distinguish balance from other concepts in the workfamily literature. According to the most widespread definition, work-family balance means "a lack of conflict or interference between work and family roles" (Frone, 2003: 145). Other approaches go beyond this and emphasize the importance of positive content and a degree of comfort between roles, besides the absence of conflict (Frone, 2003; Martinengo, 2007). In this sense, work-family balance can not only be understood as an outcome, but also as a kind of behavioural pattern of acting across roles in a certain way and trying to harmonize one's life of multiple selves. Thus, engaging multiple roles can have both negative and positive outcomes: a positive role balance refers to the tendency to engage in every role with an equally high level of effort, devotion and attention, resulting in role ease, while a negative role balance refers to the tendency to engage in roles with a low level of effort and attentiveness, leading to conflict (Greenhaus et al., 2003; Rantanen, 2008). 
More recent views of work-family balance, however, can be classified into the so-called overall appraisals approach, and the components approach. The first concentrates on the individual's general assessment of the entirety of one's life situation. As Clark (2000:751) defines it, this refers to "satisfaction and good functioning at work and home, with a minimum of role conflict". In surveys it is typically measured using one general question. A components approach emphasizes the components of work-family balance which precede it and give meaning to it (Rantanen et al., 2011; Grzywacz and Carlson, 2007). For instance, Greenhaus and his co-authors (Greenhaus-Beutell, 1985; Greenhaus et al., 2003) define work-family balance as a state whereby the individual is equally engaged in work and family life and is equally satisfied with both roles. As a consequence, both work and family life have the same priority. As a components approach they distinguish three between components of work-family balance: time balance (equal time devoted to both roles), involvement balance (equal psychological efforts and presence invested in both work and home) and satisfaction balance (equal satisfaction expressed in work and family roles). According to these authors the work-family balance is a continuum with imbalance in favour of work at one end, and imbalance in favour of family at the other end, with a state of balance in the middle of the scale, where neither the work or family role are dominant. Frone (2003) also suggests a components approach where balance consists of both work-family conflict and work-family facilitation. The advantage of this components approach over the overall appraisals model is that different aspects of work-family balance can be conceptualized, thus the phenomenon can be measured in a more complex way, producing systematically higher explanation rates than when using the overall system of appraisal (Grzywacz - Carlson, 2007). Grzywacz and Carlson (2007) include the social and relational aspect into their conceptualisation, thus exceeding the definition of work-family balance as a psychological construct only. According to them, work-family balance is the "accomplishment of role-related expectations that are negotiated and shared between an individual and his or her role-related partners in the work and family domains" (Grzywacz - Carlson, 2007: 458). In this sense, the individual tries to meet the expectations of those who are important role partners. Therefore this definition concentrates on how the individual performs in life domains, and satisfaction or the complete absence of conflict are not essential components of a state of balance. 


\subsubsection{New Approaches}

One of the new approaches in the work-family interface, so-called ecological system theory, tries to make up for the issues caused by reducing the scope to the individual level, as occurs with the aforementioned theories. According to this approach, "work-family experience is a joint function of process, person, context and time characteristics" (Grzywacz - Marks, 2000:112). Thus ecological system theory involves a holistic approach which includes micro, meso, exo and macro levels. It acknowledges that an individual's behaviour is not only affected by individual preferences but by other individuals, by the broader work and family environments and by overall societal context, values, norms, policies and culture. According to this perspective, the relationship between work and home can be both positive and negative and unidirectional or reciprocal. Also, the time dimension is added to the theory which makes the interactions between the individuals and their environment more dynamic. Accordingly, this potentially represents a more comprehensive approach to work-family dynamics (Grzywacz - Marks, 2000; Voydanoff, 2002).

Another contemporary approach is the border theory of Clark (2000). According to this author, family and work are two different domains which influence each other, but their culture and aims contrast like those of two different countries. She describes individuals as border-crossers who move between the two domains. For some individuals permeability (namely, the extent to which elements from one domain might enter the other), is feasible, while for others it might take more effort. Integration or segregation of family and work are determined by this permeability, and also by flexibility, which describes the extent to which a role can be performed outside of the typical spatial and temporal boundaries of its domain. For instance, some employees have the option to work from home, while others do not (Emslie - Hunt, 2009). Border theory suggests that flexible and permeable boundaries facilitate integration between work and family, and that when domains are integrated transitions should be easier, but at the same time interferences could be more frequent. On the other hand, when work and family domains are more segregated, transitions should be more difficult but might lead to fewer conflicts occurring at the same time (Martinengo, 2007). Thus border theory covers both positive and negative interactions and assumes interaction between the two domains, as does the spillover hypothesis. However, it exceeds the spillover hypothesis in the sense that it does not consider individuals as passive observers of 
interactions but as active participants (Dén-Nagy, 2013) who manage and negotiate work and family spheres and borders to achieve a state of balance (Clark, 2000). In addition, spillover tends to address emotional linkages (like satisfaction or expressions of frustration), while border theory acknowledges the spatial, temporal and social behavioural connections between work and family (Clark, 2000).

In connection to border theory, another important term should be mentioned: boundary management style. This refers to the "general approach an individual uses to demarcate boundaries and regulate attending to work and family roles", or namely, whether individuals are used to separating or integrating work and family domains (Kossek - Lautsch, 2012:8). Besides separation and integration of work and family, Kossek and Lautsch (2012) define a third hybrid type of boundary management style which they call 'altering'. This involves alternating between segmentation and integration. A boundary management style can be shaped by individual preferences on the one hand: some people prefer to separate their roles, for example by setting up different e-mail accounts for work and family, and trying to conduct work at the workplace and take care of family matters only outside working time. Others might prefer an integrative style of boundary management, such as texting children or partners from the office or monitoring e-mails on vacation. But even asymmetrical boundary management styles can also be observed, for example, when someone prefers to take calls from a family member while on the job, but would prefer not to receive workrelated texts when not working. In addition, one's centrality of work or family identities might shape how individuals manage work-family boundaries. The authors, however, criticize the dualistic view that individuals are either work or family centric. Instead, they suggest that many individuals have configurations that are dual-centric, and assume the existence of alternating boundary management styles. The authors also believe that positive outcomes are more likely to occur when individuals enact boundary management styles that are congruent with their personal boundary management values and preferences, therefore creating a sense of being in control of boundaries. But the boundary management style is not only dependent on personal preferences and identities, but on the organizational climate, norms, supervisor support or the flexibility and permeability of borders. In standardized work environments, members are expected to adapt to an organization's preferred way of managing boundaries. In contrast, some organizational work-family climates allow more individual customization of boundary management. 
Dén-Nagy (2013) concludes in her theoretical summary that all of the thus-far described theories have their advantages and can be used depending on the issue under investigation. Since the different work-family interface approaches have different focus and make different assumptions about the relation between work and family domains, their relevance might be different depending on the research question. For example, the type of work can determine the choice of work-family interface approach. Although the segregation hypothesis is no longer one of the most popular concepts anymore, it might still be relevant in case of blue-collar jobs where time and space flexibility or information technology usage are less optional. This is not true for white-collar jobs for which analysis spillover theory or border theory may be more appropriate.

Among the above-described middle-range theories, conflict theory has been chosen as a basic theoretical approach for this research. The reason for this is the assumption that the harmonization of work and family life might not be without problems, and the intention of this research is to better understand the possible clashes manager fathers face in their everyday lives. In addition, the concept of conflict is used as a framework to hold together the other theories that are used in the dissertation. Although conflict theory is the core and has been described as a psychological, role theory approach, the dissertation exceeds an examination of the individual level and includes the organizational and social context into the understanding as well. Moreover, I find Dén-Nagy's (2013) point about the relevance of the every work-family interface approach very important, so in the analysis and interpretation of research findings the potential occurrence of other work-family interface concepts such as work-family balance, positive interactions and boundary management styles are also taken into account.

\subsection{Work-Family Conflict}

Despite the emergence of positive interaction theories and new approaches within the work-family literature, the majority of research is still based on the assumption of conflict (Greenhaus - Powell, 2003; 2006). As already explained in the previous chapter, work-family conflict theory is based on two approaches: scarcity and role stress theory. Scarcity theory assumes that the relation of work and family can be understood as a zero sum game, since personal resources of time, energy and attention are finite. Therefore, the devotion of greater resources to one role inevitable necessitates the devotion of lesser resources to the other role (Greenhaus - Powell, 2003). Role 
stress theory assumes that if a given set of social roles impose conflicting role expectations and pressures towards a person, this can create psychological conflict and role overload for that individual. A key element of a role conflict is the simultaneous occurrence of role pressures that hinder compliance with competing role expectations. Four types of role conflict can be identified: intra-sender conflict (when the same person imposes incompatible expectations on the individual), inter-sender conflict (when demands from two or more persons towards the individual are contradictory), inter-role conflict (role pressures from one role conflict with role pressures from another role) and person-role conflict (the requirements of a given role are in opposition to the values of the individual) (Rantanen, 2008). In their influential paper, Greenhaus and Beutell (1985:77) define work-family conflict as "a form of inter-role conflict in which the role pressures from the work and family domains are mutually incompatible in some respect. That is, participation in the work (family) role is made more difficult by virtue of participation in the family (work) role". 2

Greenhaus and Beutell (1985) have posited the existence of three major forms of work-family conflict: time-, strain-, and behaviour-based conflict. Time-based conflict refers to overlapping schedules and pressures between work and family roles, due to which it may be impossible to be both physically as well as psychologically ${ }^{3}$ present within both roles. Strain-based conflict occurs when work- and family-related stressors arise that produce mental and emotional strain, due to which the demands of other life spheres are difficult to fulfil. Behaviour-based conflict refers to the different behavioural expectations related to work and family domains and the inability to adjust one's behaviour according to these expectations within each life sphere. Most of the empirical studies that use Greenhaus and Beutell's (1985) conflict definition focus on the time-and strain-based items and the findings for behaviour-based conflict are scarce, thus somewhat vague in comparison to the other two types (Rantanen, 2008). It is

\footnotetext{
${ }^{2}$ Greenhaus and Parasuraman (1999) suggest that work-family conflicts should be studied as stress episodes rather than as a chronic or on-going state. According to these authors, this perspective would enable researchers to trace a work-family conflict incident from its preliminary appraisal in the environment and study the individual's reactions, including their search for social support and the activation of different coping behaviours. This approach could capture the conditions under which environmental factors arouse psychological conflict and the usefulness of different coping and support mechanisms in resolving work-family conflict.

3 "Time-based conflict can take two forms: (1) time pressures associated with membership in one role may make it physically impossible to comply with expectations arising from another role; (2) pressures also may produce a preoccupation with one role even when one is physically attempting to meet the demands of another role" (Greenhaus - Beutell, 1985:78).
} 
important to add that these types described by Greenhaus and Beutell (1985) are not ideal typical; the different types of interference between work and family can appear simultaneously (Sebök, 2015).

Based on Greenhaus and Beutell's (1985) definition, there is also a relative consensus that work-family conflicts are bi-directional. Accordingly, work can interfere with family (work-to-family conflict) and family with work (family-to-work conflict). In quantitative research, work-family conflict has generally been measured using selfreporting scales that assess the perceived interference between the demands of the work role and the family role. Nowadays the scales incorporate the type and the direction of conflict as well (Martinengo, 2007). This idea of two-dimensionality conflict assumes that work-to-family conflict and family-to-work conflict have different sources as well as consequences (Frone, 2003). Empirical findings, however, show some reciprocal relationship between the constructs, meaning that if one experiences either of the conflict directions, one is also likely to experience the other, although it seems that the correlation remains at a modest level. Consequently, there is both overlap and unique variance between the directions of work-family conflict (Tammelin, 2009; Rantanen, 2008).

Greenhaus and Powell (2003) identified three factors that may determine the decision to participate in a work activity or a competing family activity and therefore determine the direction of the interference that occurs between work and family roles: role pressure, role salience, and role support. An individual might face an expectation that arouses a psychological force within the individual to meet such expectation. Role pressure implies that sanctions that may be positive (for compliance) or negative (for non-compliance) and can preserve the relationship with the role sender. The situation becomes complicated when simultaneous and opposing pressures occur. The authors offer an example of when a manager puts pressure on an individual to participate in a work activity while a spouse puts pressure on the individual to participate in a family activity. According to their empirical findings, individuals must then decide depending on the strength of the pressure arising from each role. Apart from external pressures, individuals may create pressure on themselves to participate in a role based on their personal conception of what it means to be an employee, team member, spouse, parent or community member. An individual may develop more demanding internal expectations in highly important or salient roles than in roles that are less central to his or her sense of self-identity. Consequently, it can be suggested that the more salient a 
role is to an individual, the more time and emotion are invested in the role. Although, as Kossek and Lautsch (2012) state, it would be a simplification to describe individuals as solely family-or completely work-centric. Greenhaus and Powell (2003) further explain that in addition to external and internal pressures, support for the individual may influence work-family participation decisions. Support offered by role senders in either the work or family role might enable participation in the other domain. At the same time, however, this support might also strengthen commitment towards the role sender. For example, having a supportive manager can lead to more freedom in prioritizing family activities, but on the other hand employees may choose to engage in work activity as a way of paying back their manager for being supportive. Similar research results and measurement related to the antecedents and consequences of both work-tofamily and family-to-work conflict will be described in detail in the chapter on former empirical findings (Chapter 3).

The role theory side of work-family conflict can be further specified since it has a broad psychological literature. The aim of this dissertation, however, is not to delve in depth into the psychological concept of work-family conflict, but to use the concept as a starting point and framework for sociological subjects of interest such as gender and organizational dimensions. The sociological terms and concepts connected to workfamily conflict theory will be described in the next subchapters $(2.3,2.4,2.5$ and 2.6 subchapters) along the three conflict types: time-based, behaviour-based and strainbased conflict. The relevance of time-based conflict will be emphasized in the context of the post-Fordist time regime, flexibility, and visibility. In relation to behaviour-based conflict I emphasize the contradictions between the expectations towards involved fatherhood and the demands of managerial roles. Regarding strain-based conflict, the relations between stress, psychological and physical well-being, social support and control will be introduced. In order to go beyond the individual level, the relationship between employees and organizations will be briefly described with a focus on the employees' ability to claim a work-family balance. Finally, all of these aspects will be placed in the national context by describing the Hungarian gender and work-family environment. For better understanding, a figure (Figure 1) is included to describe the structure of theoretical concepts: 


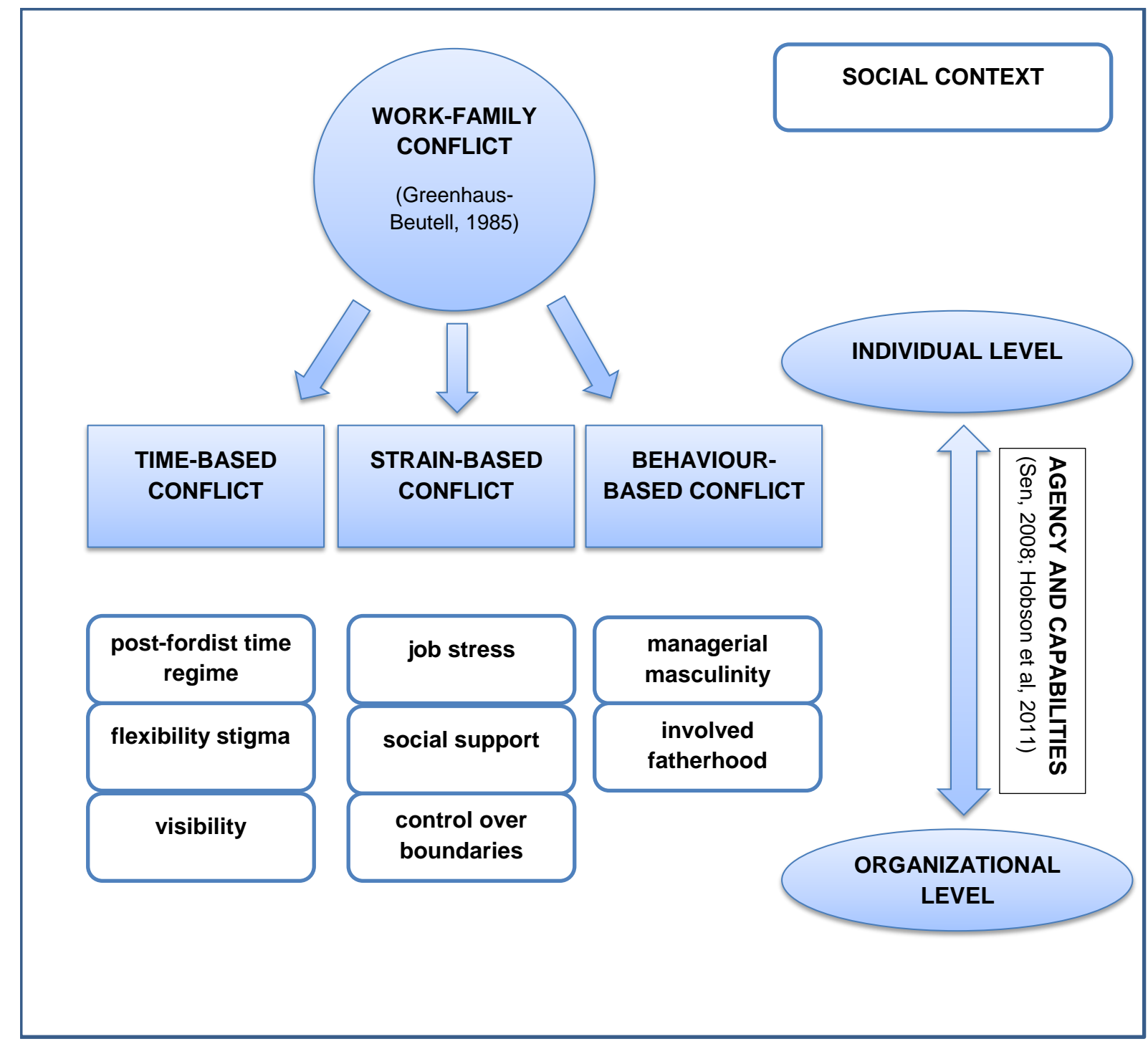

Source: Author's construction

\subsection{Time-Based Conflict and Post-Fordist Time Regime}

The high performance organizations which now exist, especially in globalized working life, are increasing their expectations towards employees regarding time, energy and commitment. The constant and fast-paced change of organizations, the more demanding intensified working practices and environments result in feelings of pressure, a lack of time and general "busyness" (Lewis et al., 2007). Employees are provided with smartphones and laptops so they are available and capable of working from home as well. New kinds of information technology, the spread of networks and the increasing number of transnational companies have dramatically changed the use of working time and the issue of flexibility (Hill et al. 2004; Kvande 2009; Burke 2000). In 
service sector professional jobs, the knowledge each worker possesses is the main resource or capital of a company. This, in turn, requires a certain type of work organization that is able to give employees a great degree of autonomy so they can use their knowledge in both a creative and an efficient way (Kvande 2009). All of these changes have resulted in the emergence of a new, flexible time regime which promises greater autonomy, in sharp contrast to the traditional, industrial Fordist style of time management with its standardized working hours and fixed time schedules which draws a clear boundary between work and free time. Formal contracts that regulate working times are often being replaced with time norms built on moral expectations and total commitment. These have led to the emergence of so-called 'boundless' time cultures which are highly typical of knowledge companies which require a high level of commitment. A person is responsible for the maximal fulfilment of their work - which primarily consists of project-based tasks -, for which he or she decides how much time is needed (Kvande 2009; Tausig - Fenwick 2001; Kelly et al. 2010).

Companies functioning in the high-paced post-Fordist environment are often labelled greedy organisations (Coser, 1974) which seek exclusive and undivided loyalty from their employees. Although a professional career is often regarded as "individualized" in the post-Fordist time regime - namely, in the sense that employees are independent from institutions and are focused on their own interests and less on loyalty (Wajcman - Edwards, 2005) - greedy organisations are able to generate commitment from employees in three ways: firstly, they make significant demands on their members' time and energy. Secondly, they offer a position of status by creating an aura of exclusivity around the institution and by putting pressure on individual members to weaken their ties outside the organization. Thirdly, they build close links with the social identity of their members through the elements of this exclusivity (Burchielli et al., 2008).

This observation accords with the ideal employee definition of Joan Acker (2006; 2011). The ideal employee is a worker who exists only for work, and has no other claims. As Acker (2011:67) says "He is expected to be at work at set times, focused only on the tasks at hand, responsive only to demands of supervisors, available for long working hours, and unhampered by other responsibilities, such as children and housework. This is the ideal, unencumbered worker. The encumbered worker, most often a woman, does not fit the ideal assumptions very well." Consequently, work is organized around the image of a white middle-class man (Acker 1990, 2006). The work 
devotion schema of Mary Blair-Loy (Erin - Blair-Loy, 2014; Williams et al., 2013) describes the same deep cultural assumption that work deserves undivided and intensive allegiance. This schema is "both coercive - many workers feel forced to comply - and seductive - workers may also believe that a strong work ethic helps form their sense of self and self-worth" (Williams et al., 2013:211). The work devotion schema can be also understood as an implicit contract between the employee and the company, assuring the employee that his or her sacrifices of time and energy will be honoured. Therefore the unspoken expectation that employees will minimize the time they spend with family is institutionalized in company practices ${ }^{4}$ (Williams et al., 2013; Erin - Blair-Loy, 2014).

Therefore the secret behind greedy organizations within a post-Fordist environment is the hidden mechanisms: in a boundless time culture, work is internalized, always present in the mind of workers without the need for the employer to control the employee. The disciplinary processes of empowerment and individualization make professional and managerial employees themselves the driving force behind a culture of long working hours. Professional employees with a higher level of work autonomy are seen as 'trusted workers' who are felt not to need close supervision (Williams et al., 2013). It may appear that it is the individual's responsibility to say "stop: enough!" but this does not happen because the pressure is too great, on the one hand, and knowledge work is a major source of elite status and identity on the other (Lewis et al., 2007; Kvande, 2009). In professional and managerial positions, the exclusivity and prestige associated with a job can be seductive to employees. These jobs are increasingly complex, intellectually absorbing and exciting, requiring a high level of discretion and creativity that can easily become the centre of identity and emotional life

\footnotetext{
${ }^{4}$ Blair-Loy’s conception (cited by Halrynjo - Lyng, 2009) analyses work-family conflict in terms of 'cultural schemas': frameworks for viewing, filtering, understanding and evaluating what we know as reality. Blair-Loy turns work and family commitments into compelling 'schemas of devotion': as already explained, the work devotion schema demands that one dedicates an immense amount of time, commitment and emotional allegiance to one's firm or career, which in return provides not only an income but a sense of identity and belonging. The family devotion schema prescribes a model of parenthood that is intensive, emotionally absorbing and centred around one's children. Each schema reproduces work and family as competing and mutually exclusive sources of devotion that gradually become naturalized and unquestioned. The importance of this concept is that it exceeds individual preferences about prioritizing work or family and understands the work-family conflict to be a socially constructed phenomena with norms, constraints and hidden mechanisms.
} 
(Wajcman - Edwards, 2005). Moreover, as Hochschild (2001) concludes in her book The Time Bind written about the American Amerco company, in some respects employees choose to focus on work because it can offer more satisfaction and reward and be more manageable than often-messy personal relationships at home. Thus, greedy organizations are also 'seductive' organizations at the same time, and as a result home becomes work and work becomes home, as the subtitle of the book implies ${ }^{5}$.

Moreover, there are no regulations or restrictions which can prevent professional employees from working long hours, if they so choose (Allard et al., 2007). Thus, work devotion becomes a 'class act' - a way to show elite status, both justifying and fuelling very long working hours (Williams et al., 2013). As a result, how to harmonize work and family can appear to be a personal responsibility, an individual's 'choice', while the role of the organizational system is not emphasized, even if choices are socially embedded (Lewis et al., 2007).

Research findings suggest, however, that a high-performance environment requires that companies support the work-family balance and family-friendly initiatives for their employees (Van Echtelt et al., 2009). The most typical family-friendly arrangements that companies offer are usually based on flexibility. Flexible initiatives primarily include flexible working hours such as flextime, flex leave, part-time options or flexible working locations (through telecommuting) (Allard et al. 2007). Flexible working hours (other than the right to work part-time) are not inscribed in EU Law, but are rather put forward as an example of good company practice for promoting the workfamily balance of employees (Hobson et al., 2011). Thus, at first sight flexibility seems to be the solution to work-family balance problems in a high-paced, boundless working environment. The solution is not so simple, however. Empirical research suggests that access to flexible working options does not always correlate with a better work-family balance (Tausig - Fenwick, 2001). Flexibility may also have unexpected side effects, since without clear boundaries between work and private life work can easily continue at the expense of family life in the case that employees work even longer hours than they would without such arrangements (Kvande, 2009; Tammelin, 2009; Kelly et al., 2010). Hochschild (2001), in her above-mentioned case study, also draws attention to the fact that flexible working opportunities ultimately lead to more overtime.

\footnotetext{
${ }^{5}$ Hochschild (2001) also describes how efficiency techniques from the workplace are increasingly being applied to the home, for example in how 'quality time' with children is rationalized, while corporations try to make the workplace more homely in order to produce a strong culture of commitment.
} 
The other problem with flexible and family-friendly opportunities is that although they are seen as an indicator of an organization's responsiveness to employees' work-family concerns, they often exist only on paper for public relations reasons, and real usage rates are low (Williams et al., 2013). Low usage rates stem in part from fears of flexibility stigma and career penalties, since the use of flexibility policies might result in wage penalties, lower performance evaluations and fewer promotions. But even in organizations that have made real effort to maintain their employees' work-family balance, these programs have not come close to dislodging the norm of the ideal employee and expectations about devotion to work because informal practices often stigmatize the use of these policies (Williams et al., 2013; Erin - Blair-Loy, 2014).

Moreover, stigmatization stemming from the use of flexible working arrangements "is rooted in gender stereotypes and class divisions" (Williams et al., 2013: 228). Although flexibility and family-friendly policies are formulated in a genderneutral way, they implicitly target women in the first place (Burnett et al. 2010). Mothers are even more exposed to flexibility stigma since they are seen as being less committed to work, thus violating the work devotion schema (Erin - Blair-Loy, 2014). Fathers are also regarded as less predictable in the case that they take advantage of these flexible working opportunities. Moreover, the use of these arrangements is more costly for men. Even using modest work-family policies, such as taking a few days of sick leave to care for an ill child, can trigger penalties for fathers (Erin - Blair-Loy, 2014). The reason for this is that having a managerial position requires men to be irreplaceable at work (Kvande 2009; Halrynjo - Lyng 2013). All these hidden assumptions are built upon the concept of the ideal employee (Acker, 2011), as explained above. In this sense the hegemonic forms of masculinity ${ }^{6}$ are still associated with work that entails long hours and behaviour and demonstrates the prioritization of such work. Those men who construct masculinity through flexible working hours might be construed as men who have rejected the constraints of hegemonic masculinity (Thompson, 2010). If professional men do not show complete dedication to their careers and do not consistently perform, they risk later career advances and success. Although the risk and career costs might seem to be gender neutral, the ongoing discourse seem to minimize the consequences for mothers, while at the same time accentuating the risks to fathers.

\footnotetext{
${ }^{6}$ Hegemonic masculinity is the term used by Connell (Connell, 2009) to describe the pattern of practice (i.e., things done, not just a set of role expectations or an identity) that have permitted men's dominance over women, as well as subordinated masculinities, to continue (Connell - Messerschmidt, 2005).
} 
In the background stands the unspoken message that men's careers are still regarded as more important than women's (Halrynjo - Lyng 2013).

As can be seen, a long-hours culture can demand that employees who are interested in career mobility work hard and demonstrate commitment and loyalty through presenteeism; namely, by visibly working long hours at the office, regardless of whether long hours are needed to achieve performance goals (Allard et al., 2007). Another term used regarding such visibility is 'face time', defined as the employee's "physical time at the workplace that is observed by co-workers, supervisors, and customers. Face time includes face-to-face interaction, working in the presence of others, and "being there" at work" (Van Dyne et al., 2007:1125). In greedy organizations, time seen at work is equated with productivity. Consequently, many professionals experience pressure to manage their face time and to be present (Van Dyne et al., 2007), although thanks to advanced information technology and equipment, personal presence is not always necessary for accomplishing work. It is easy to realize that the compulsion of visibility and face time stands in opposition to the idea of flexible working arrangements.

Although many corporations have introduced flexible working policies, these initiatives do not usually challenge deeply held convictions about how work should be carried out, the nature of the ideal employee, the deep-rooted cultural values of work devotion, visibility, or the inevitability of unquestioned working hours (Lewis et al, 2007; Williams et al., 2013). As long as taking up family-friendly policies remains an individual choice, the risk of being considered a time deviant, and thus being marginalized at work remains (Kvande, 2009). Kvande (2009) suggests as a remedy the use of state regulations and standard solutions, since collectively supported and collectively taken-advantage-of provisions could decrease the potential costs and would provide fathers with the legitimacy needed to better define limits to work. Similarly Halrynjo and Lyng (2013) assume that more gender equality would minimize the costs of lagging behind in competition. They suggest extending the current quota for fathers in Norway, which would legitimize fathers' absence from work, thus make fathers more 'replaceable' at work and 'irreplaceable' at home. 


\subsection{Behaviour-Based Conflict, Managerial Masculinity and Involved Fatherhood}

Behaviour-based conflict is the least studied conflict type since it is more abstract and difficult to specify than time-based or strain-based conflict (Rantanen, 2008). Greenhaus and Beutell (1985) offer the example of male managers to demonstrate incompatible expectations regarding behaviour in different roles. According to these authors, the male, managerial stereotype emphasizes self-reliance, emotional stability, aggressiveness and objectivity. Family members, on the other hand, may expect a person to be warm, caring, open and emotional.

As explained in the previous chapter, the model of the ideal employee or the work devotion schema highlights both the organizational and social expectations for men to consider work the main element of their life, especially those men in professional and managerial occupations. The traditional breadwinner model, whereby the man is responsible for obtaining paid work and the woman's duty is unpaid home and care labour, accords completely with this assumption. Under this interpretation, being a good father, unlike being a good mother, is not seen as culturally incompatible with being a good employee. It rather works to the contrary: being a good provider is seen as an integral part of being a good father. In professional positions fathers are often considered to be more committed to work than men without children and are therefore more likely to be recommended for management or offered higher salaries (Williams et al., 2013).

This is not the case, however, in those situations where the traditional breadwinner model is not followed. The notion of man as the ideal breadwinner is contested by the ideal of the involved father who is committed to caring and family responsibilities (Williams et al., 2013). The rise of the ideal of the nurturing father is not something to be understood in the frame of the ideal employee or work devotion schema, since expectations towards men are different according to these concepts. While the ideal employee and work devotion schema expect men to focus primarily on their work, thereby neglecting care-related responsibilities, the ideal of involved fathering expects fathers to take an active role in their child nurturing and care. These contrasting expectations might help with understanding why men now report greater levels of work-family conflict than before (Williams et al., 2013; Kvande, 2009). 
Based on this observation it can be assumed that the gap between the ideal manager and involved father might be a significant source of conflict in the life of those male managers who identify themselves with the ideas of involved fatherhood. To better understand this discrepancy, in the following the literature about managerial masculinity will be briefly summarized along with the theory of caring and involved fatherhood on the other hand.

\subsubsection{Manager Men in Organizations}

For a long time organizational structures and processes were viewed as gender neutral (Collinson - Hearn 1996). The literature on management and organization theory has consistently failed to question its gendered nature. One reason for this is that gender is difficult to see when only the masculine is present. In most cases, men in organizations are seen as the reference and to represent human in general (Acker 2010; Collinson - Hearn 1996). But organizations are gendered and bear masculine values. The masculine character of management and managers seems to be being taken for granted, neglected, and thereby reproduced and reinforced (Collinson - Hearn, 1996). Managers have become a key group for understanding modern masculinities and are significant social groupings in public patriarchies (Hearn 1992).

The concept of power and control is a key feature of both organizational theories and masculinity studies. Heretofore, they have been studied separately, however: the first in relation to hierarchy and management, and the second related to gender and men. In the former, managerial power and control has generally been examined without a gender lens, while in the latter men's power, control and ideology have been emphasized without including organizational approaches. Thus, most of these critical studies have not explicitly considered the interrelations between men, power and management. This kind of dualism - between critical studies of either management, or men - still exists (Collinson - Hearn, 1996).

As a result, studies on masculinities and management have tried to merge the literature of power, management and masculinities. It is recognized now that gender and gendered power relations are major and defining features of most organizations and managements (Broadbridge - Hearn, 2008). The asymmetrical power relations of hierarchies and the gendered power of men managers are more contradictory and heterogeneous than they first appeared. Power relations are changing, fragmented, shifting and incomplete (Collinson - Hearn, 1996). Control has a contradictory nature 
which is difficult to see due to the assumption that being in control is a central characteristic of men and dominant masculine identities. Men can nevertheless feel trapped when they realise that they may not be in full control of others, of the environment or themselves. Male uncertainty may be growing with social and economic forces such as new technology, unemployment, feminism or equal opportunity initiatives and intensified class and status divisions within organizational hierarchies, all of which have been shown to threaten masculine identities (Collinson - Hearn, 1994). It can be seen that the capitalist economy which constructed men as wage earners and reshaped masculinity by linking gender identity with work is the same process which has made new masculinities vulnerable. The global economy, with its economic downturns, crisis and unemployment as well as booms, can undermine masculinities identified with work (Connell 2005).

The changes of the last few decades have significantly affected organizational masculinities. One determining change in Western capitalist organizations has been the increasing appearance of financial capital. Formerly, the older generation of managers had a close relation with the process of production and identified themselves closely with the firm and the quality of the product. New managers, however, are more oriented towards accountancy and profit, and are less interested in technology (Connell - Wood 2005; Connell 2009; Broadbridge - Hearn 2008). Connell (2006a) identifies two important changes: through an investigation of Australian public sector worksites this author firstly finds that there has been an increase in 'flat' organizations (i.e. reductions in the number of levels of management). This has reduced the distance between managerial and non-managerial staff and brought senior management socially closer to employees. These finding agree with Acker's (2006) observations from the analysis of Swedish banks in the late 1980s, on the one hand, and a welfare agency on the other. Acker argues that these organizations have removed some layers of middle management and delegated some decisions to lower organizational levels. The aim of these changes was to get rid of the inefficiencies of old bureaucracies, to reduce hierarchy and inequality, and finally, to empower lower-level employees. In reality, employees found themselves with greater responsibilities, but similar levels of control and salaries; consequently, formal hierarchies remained the same. The other basic change Connell (2006b) observed in bureaucracies was a shift from authoritarian management styles toward more consultative and inclusive styles of management. The move towards more flexible corporate structures places more emphasis on attitudinal, behavioural and 
personality factors. Leadership is now concerned with fostering shared visions, shared values, shared directions, and shared responsibility, requiring a softer edge and more empathy (Wajcman - Edwards, 2005).

In accordance, Hearn (1992) considers bureaucracies mixtures of patriarchal and fratriarchal organizations. In these so-called organizational hybrids, men may meet with each other in fratriarchies, yet relate to each other through the processes of patriarchal and hierarchical authority. Even if fraternity involves bonds between equals, its organizational forms are still characterized as selective, competition-orientated and hierarchical. In this sense, hierarchy reinforces the dominant models of masculinity that are readily associated with men of power. But the tension between hierarchy and group solidarity can be seen as the tension between two models of "doing masculinity" (Morgan 1996). Thus, the phenomenon of fraternity shows that the assumption that men act only in order to differentiate themselves from others by showing superiority and obtaining control over others is misleading. While they are encouraged to be self-reliant, innovative, and make their own career choices, at the same time they are expected to be good team players and to conform to company norms (Wajcman - Edwards, 2005).

Since the 1990s there has been growing recognition of multiple forms of managerial masculinities. Collinson and Hearn (1994), for instance, argue that hegemonic masculinity may appear in a number of different ways. They call these forms 'masculinities' and propose that different men and different organizations adopt different masculinities as normative behaviours, instead of there being just one kind of man. "Accordingly, different forms of masculinity are embedded in relations of power, and particular forms may be characterized as 'hegemonic' or 'subordinate' in relation to each other. In turn, these masculinities are not fixed, but continually shifting. They have been shown to be culturally and historically contingent" (Collinson - Hearn 1994:6).

Among all of the changes in organizations, special attention has been paid recently to the move away from nationally based, single organizations to transnational, post-bureaucratic multi-organizations with looser structures, dynamic networks and project organization (Broadbridge - Hearn 2008; Kvande, 2009). These institutions have their own gender regimes ${ }^{7}$ and may form the basis for new configurations of masculinity. Connell calls these transformed patterns of masculinity transnational business masculinity (Connell - Wood 2005). As greedy organizations try to bind

\footnotetext{
${ }^{7}$ Gender regimes are patterns in gender arrangements within institutions. They are therefore a typical feature of organizational life, and are not static but changeable (Connell, 2009).
} 
employees to the corporation, the new transnational forms of masculinities seem to be more independent from these organizations. Connell (2006b) claims that the new managerial career model does not build on total commitment and loyalty. Instead, it involves movement between organizations instead of lifetime career commitment (namely, climbing up the ladder within the same organization). In effect, one's life is constituted as an enterprise, something treated to be managed. This means the managing of one's emotions, personality ${ }^{8}$, and body. This can be seen as a shift from a bureaucratic to an entrepreneurial style of management. While these changes represent a kind of 'freeing' of managers from institutional and normative constraints, they also make them more susceptible to risk (Wajcman - Edwards, 2005).

The labour process described above is not healthy, involving long hours, high stress, sedentary work, and frequent travel (Connell 2005). What still keeps people in high-stress, unhealthy, insecure jobs is not only money, but the access to power. The pull of economic, social and symbolic power associated with male management reinforces an individuals' commitment to and engagement in business (Bowman, 2007). In addition, working long hours is seen as a 'heroic activity'; a manly test of physical endurance. The successful enactment of this masculinity involves displaying one's exhaustion, physically and verbally, in order to convey the depth of one's commitment, stamina and virility ${ }^{9}$ (Williams et al., 2013).

\subsubsection{Involved Fatherhood, Changing Masculinities}

After briefly summarizing the main trends in organizational and managerial masculinities, let us move on to the topic of fatherhood, where the expectations attached to involved fathers are the most controversial as regards ideas about ideal employees and managerial masculinities.

In the past few decades some significant changes have occurred regarding fatherhood, primarily in Western countries. The issue of the necessity of making men partly responsible for housework and childcare emerged as early as the 1950s. This approach was regarded as an appropriate way of promoting gender equity and creating family solidarity (Nagy, 2008). This new idea of fatherhood is very different compared

\footnotetext{
${ }^{8}$ Managers are also required to reveal themselves as human beings with the associated weaknesses and vulnerabilities - which under conventional managerial practices in more bureaucratic regimes can be hidden (Kerfoot - Knights 1996).

${ }^{9}$ Accordingly, flexibility stigma for manager men stems from the idea that a working man who places stress on fulfilling caregiving responsibilities is less of a man (Williams et al., 2013).
} 
to that which existed about fathers in the 1950s to 1970s, who were presented as occupying a more distant breadwinning role. Today it is no longer enough to be rational, goal/means oriented, career-oriented, and disciplined: neither 'earning as caring' nor having a family photo on the office desk is sufficient to validate a man as a good father. The new father is more emotionally involved, more nurturing, and more committed to spending time with his children as well as willing to share the joys and work of caregiving with mothers (Wall - Arnold, 2007). The literature suggests that the new concept of involved fatherhood has affected the understanding of masculinity in general, and we can also observe the rise of the concept of the new man; namely, a vision of a new care-oriented masculinity (Johansson - Klinth, 2008). This careoriented masculinity includes many other relations besides fatherhood - for example, friendship or partnership (Holter, 2007).

We can observe initiatives at the supranational level (e.g. the European Committee's Gender Roadmap) which are designed to invite men with the help of social policies to more actively participate in family responsibilities, and to support the notion that men and women should be able to engage in both employment and care-giving (thus, to 'de-gender' care). The Gender Roadmap, which deals with gender equality, includes a dedicated section about involving men (Nagy, 2008). But there are also many examples of national solutions for improving the involvement of fathers, many of which come from Nordic societies which is regarded as a guiding light in terms of the support it offers for involved fathering. From the 1990s in particular, there has been a rapid expansion in the Nordic countries of both parental leave and flexible working provisions targeted at fathers (O'Brien et al, 2007). The reasoning behind the parental leave scheme is not only to facilitate opportunities for women to combine work and family life, but to ensure that these responsibilities are shared between women and men and to strengthen the father-child relationship. This can be regarded as a development towards a father-friendly welfare state (Kvande, 2009). Involvement of the father influences a child's development positively, since they can feel that both parents are available and able to take care of them. In addition, involved fathering has positive effects not only from the child's viewpoint but for the fathers themselves as well (Eräranta - Moisander, 2011; Duyvendak - Stavenuiter, 2004). To put it simply, paternity leave can be considered a form of statutory entitlement that enables a father to be absent from work for a period of time when a child is born. By contrast, parental leave is the statutory entitlement to be absent from work after initial early maternity and 
paternity leave. Until relatively recently, parental leave has tended to be a family-based public policy provision, largely available equally to parents. Father-targeted parental leave (the so-called daddy quota ${ }^{10}$ ) involves periods of time that only fathers can take off. This quota can be seen as a welfare system-directed means of forcing fathers into taking part in early childcare and adopting a new understanding of their child's needs and feelings by being present for routine activities ${ }^{11}$. This has led researchers to suggest that we are moving toward the social ideal of father as co-parent, which involves changes in the hegemonic structure (Forsberg, 2007; Wall - Arnold, 2007; O’Brien et al, 2007).

As with managerial masculinities, it is important here as well to highlight that we cannot distinguish one universal form of fatherhood. Based on historical and social science research, the image of fatherhood has fluctuated throughout the centuries and cannot be said to have evolved in a gradual, linear fashion from a more distant and authoritarian mode to a more involved and nurturing one. Cultural understandings of both fatherhood and masculinity are multiple, fluid, and shifting. Fatherhood is not once and for all a given state, but rather a continually changing one; it can be understood as competing discourses and desires that can never be fully and neatly shaped into a single identity, and which involves oscillation back and forth between various modes of subject positions (Forsberg, 2007; Wall - Arnold, 2007). It may also be true that there is a disconnect between normative expectations and actual behaviour. Fatherhood might have undergone more changes in culture than in practice. For this reason, the general public may conceive of fathers as being more involved and nurturing than they truly are (Wall - Arnold, 2007). Some research (Forsberg, 2007; Johansson - Klinth, 2008) also claims that there is a conflict between the discourse which connects the father's involvement with gender-equality and that which connects it with child-centeredness. Paternal involvement does not necessarily imply gender equality. In fact, the tendency may be for fathers to spend more time playing and talking with their children than engaging in childcare and housework. According to these findings, men's orientation towards children may be more a question of their 'picking out the good bits', than of a

\footnotetext{
${ }^{10}$ Such individualized, non-transferable entitlement to parental leave was first made available to fathers in 1993 when Norway became the first nation in the world to offer a four weeks father's quota that could not be transferred to mothers (Haas - Rostgaard, 2011).

${ }^{11}$ In Norway, contrary to early expectations, the scheme was very popular, and the number of fathers who took advantage of it increased dramatically from 4 percent in 1993 to 90 percent in 2003 (Kvande, 2009). This means the normalization of the use of the daddy quota must be considered an adaptation to the state's activities of gendering men as fathers. The success of the scheme lies in its non-optional nature since it is a 'use it or lose it' benefit (O'brien et al, 2007).
} 
radical transformation of masculinity. Thus we can differentiate between child-oriented masculinity and gender-equal men (Johansson - Klinth, 2008).

There are at least three theoretical approaches to explaining why men tend to participate more in 'fun' or highly visible aspects of child care while women continue to do the more quotidian, labour-intensive tasks: relative resources, time availability, and gender ideology (Rehel, 2013). The first two approaches, which are basically rational choice theories based on economics models, emphasize the rationality in the division of paid and unpaid labour, positioning housework as something undesirable that both men and women attempt to avoid. The relative resources explanation states that the partner who brings the most resources to a relationship, often in terms of income, has the most power, enabling the partner to opt out of unpaid labour. Similarly, the time availability explanation suggests that child care and domestic labour should be left to the person who has the most time available. Since, on average, men still have more opportunities on the labour market and can obtain higher wages than women, from a rational point of view it would be disadvantageous for men to do less paid work and women more. As a result, women tend to engage in more unpaid labour (Duyvendak - Stavenuiter, 2004; Rehel, 2013). Nevertheless, rational choice theories cannot explain why the asymmetric division of unpaid labour exist in those households where men and women have the same opportunities on the labour market (Duyvendak - Stavenuiter, 2004). According to Hochschild (1989), men with well-compensated wives have difficulty maintaining their masculine image, thus they tend to resist changes that challenge 'appropriate' gender attitudes and their male gender identity. The gender ideology approach thus explains how attitudes around who is responsible for doing the paid and unpaid labour shape how these forms of labour are distributed across couples (Rehel, 2013).

In contrast to the previously described approaches, Rehel (2013) offers the alternative typology of Lamb and colleagues (Lamb et al., 1987) which classifies the ways a parent might be involved in childcare: specifically interaction, availability, and responsibility (or as Rehel (2013) refers to them: engagement, accessibility and responsibility). This model captures various forms of involvement, from shared activities like reading and playing (interaction/engagement), to being present or accessible to the child whether or not direct interaction is occurring - for example, preparing a meal while the child does homework (availability/accessibility), to planning and scheduling around the child; for example, making appointment with the baby-sitter or determining when the child needs new clothes (responsibility). Rehel's (2013) 
interview results show that fathers in the United States and Canada have significantly increased their levels of engagement and accessibility but have changed little in terms of responsibility. Responsibility for children is consistently understood as one of the most fundamental elements of good mothering, often invisible, that adds to women's share of labour in significant ways.

\subsection{Strain-Based Conflict, Job Stress and Social Support}

According to the definition provided by Greenhaus and Beutell (1985:80), the third type of work-family conflict, strain-based conflict, "exists when strain in one role affects one's performance in another role. The roles are incompatible in the sense that the strain created by one makes it difficult to comply with the demands of another". Strain-based conflict can be approached primarily using psychological and health explanations since the core element, stress is associated with negative psychological and physical outcomes. "Stress is defined as an event or situation that is perceived as threatening, demanding or challenging." (Bell et al., 2012: 26). Two elements of the stress process can be distinguished: the actual interchange between the person and the environment; and the person's responses over time to the stress. Long-term stressors cause more severe health problems than short-term stressors (Bell et al., 2012). Studies have explored the connection between stress and work-family outcomes, including not only conflict situations but very frequently work-family spillover (Grzywacz - Marks, 2000).

Besides generally dealing with stress, numerous studies focus on the stress which stems from work; namely, job stress. Job threat stress, for example, can be understood as qualities of work experience which induce feelings of being overwhelmed or nerve-wracked. Another type of job stress, job pressure stress, is on the other hand the perception of work as demanding or time-pressured (Bell et al., 2012). It can be seen that job stress is quite strongly related to increasing work expectations, the pressure of market-driven competition and the demand for growth and efficiency, just as described in the previous chapters. As a result of the emergence of flat organizations and empowerment, the job of managers has become more complex (Mayo et al., 2011), and due to the high demands on their performance they have to shoulder more responsibility. The extra skills and responsibilities required for more challenging and rewarding professional jobs may well produce heavy workloads that are in turn associated with stress and lower job satisfaction. Moreover, the individualization of 
careers described in the prior chapter generates higher level of risks and less job security which might also cause frustration and pressure in the lives of managers (Wajcman Edwards, 2005).

Nowadays, work-related stress has been identified as one of the largest problems in the European Union working environment (Bell et al., 2012), since job stress has been proven to be correlated to negative effects on employees' psychological and physical health, representing a large emotional cost to employee wellbeing and putting a considerable financial burden on organisational performance (Bell et al., 2012; Burke, 2000). Rising levels of stress and sickness-related absences question the sustainability of current values and ways of working (Lewis et al., 2007). According to Greenhaus and Beutell (1985), work stressors can produce symptoms of strain such as tension, anxiety, fatigue, depression, apathy, and irritability. The link between work-family conflict and job stress also works in reverse, since high levels of work-family conflict and a poor work-family balance correlate to reductions in job satisfaction, lower organisational commitment, lower productivity and performance, lower levels of career satisfaction and success, higher absenteeism and increased intention to quit, as well as employee burnout, poorer physiological and psychological health and diminished family functioning (Bell et al., 2012; Eek - Axmon, 2013; Burke, 2000).

A crucial factor in reducing job stress is social support which is defined by Kossek and co-authors (2012) as both the individual's belief that they are being loved, cared for and valued and the perception of having access to direct and indirect helping relationships which provide resources such as the communication of information, emotional empathy or assistance. Social support can be also defined as "interpersonal relationships and social interactions that help to protect individuals from the effects of stress" which influences psychological well-being (Aycan - Eskin, 2005: 454). Not only the actual availability of social support but also the perceived likelihood of receiving support can reduce stress (Aycan - Eskin, 2005).

One specific type of social support is spousal support which plays a significant role in how individuals can manage stress that stems from work and family demands (Grzywacz - Marks, 2000). "Spousal support is the help, advice, understanding, and the like that spouses provide for one another" (Aycan - Eskin, 2005: 455). It can take the form of emotional support, including emphatic understanding and listening, affirmation of affection and advice and concern for the individual's well-being, while instrumental support relates to help with household chores and childcare. Thus instrumental spousal 
support eases the burden of family demands, while emotional spousal support enhances feelings of self-efficacy (Aycan - Eskin, 2005). Spouses who get higher levels of support generally report greater satisfaction and experience better longitudinal marital outcomes than do unsupported spouses. Regarding the gender aspect of spousal support, there is a general conclusion within the literature that a 'support gap' exists in marital relationships, in that men receive more support, and more helpful support than women do (Neff - Karney, 2005). Kanter (1977), in her influential book Men and Women of the Corporation, uses the term 'corporate wife' for those women who provide technical, social and symbolic support to their husband's corporate career invisibly from the background. Their support is 'invisible' because their roles are played out outside the corporate environment, although their help is crucial to building the husband's status and prestige. Consequently, husbands and their employers benefit directly from their wives' unpaid social and domestic work (Wajcman - Edwards, 2005).

Another important type of support is workplace social support, which is defined as "the degree to which individuals perceive that their well-being is valued by workplace sources, such as supervisors and the broader organization in which they are embedded, and the perception that these sources provide help to support this wellbeing" (Kossek et al., 2011: 291) The authors further distinguish 'content general' and 'content specific' workplace support, where the first relates to general work support (namely that employees perceive that supervisors or employers care about their global well-being by providing positive social interaction or resources), while content-specific support involves perceptions of care and the provision of resources to reinforce a particular type of role demand. In the context of the work-family interface, contentspecific support refers to the work-family initiatives the company or supervisors offer to employees to help them harmonize work and family demands.

In accord, perceived psychological control is also found to reduce the strain derived from work and family harmonization (Kossek - Lautsch, 2012). As previously described, due to the post-Fordist time regime, professional and manager employees who work in a globalised, knowledge-based environment face not only greater work demands (Burke, 2000), but they experience a blurring of boundaries as well. As the boundary management approach explains, the perception of control over boundaries can be crucial to individual well-being (Kossek - Lautsch, 2012), as being in control of one's preferred boundary management strategy has been shown to be more important for reducing work-family conflict and job stress than whether the individual prefers to 
segment or integrate work and personal life (Mellner et al., 2014). Therefore, as previously explained, organizational background and workplace support is crucial in the context of whether employees have the freedom to practise their preferred strategy regarding boundary management and the work-family balance (Kossek - Lautsch, 2012). The next chapter further discusses, in detail, how the organizational environment can influence employees' agency and capability to create appropriate work-family balance.

\subsection{Organizations, Agency and Capabilities}

Hobson and her co-authors (2009; 2011) use Sen's (2008) agency and capabilities framework to gain more complex understanding of work-family balance than simply analysing at the individual level. They find the agency and capabilities framework to be a dynamic tool which seeks to explain the lack of change in approaches to gender, family, and work. "The capability paradigm provides a multilayered approach to agency by (1) embedding individual agency into specific institutional settings, (2) acknowledging differences in situated agency (variations in resources and means), and (3) constructing a theory of agency that recognizes the importance of the cognitive level of agency: whether one can convert resources into who you are and what you do" (Hobson - Fahlén, 2009: 218). This means that this approach exceeds the individual level by including the institutions, rules, and informal norms collectively held by others, and by analysing how these influence the individual's access to resources as well as the perception about their own freedom to choose. They consider work-family balance to be something of value which can enhance general quality of life when achieved. Hobson and her co-authors (Hobson et al., 2011) do not intend to define what the ideal work-family balance is. Instead, the capabilities paradigm focuses on the "possibilities for converting resources into substantive freedoms to make choices and the constraints (institutional and societal/normative) that lead toward work-life imbalance and its consequences" (Hobson et al., 2011:171).

Conversion factors (social rights; gender equality norms), situated agency (resources and assets) and workplace organizational culture determine how institutional resources are converted into agency, since these three elements together lead to a socalled sense of entitlement to make claims for work-family balance, and therefore to higher agency freedom (Hobson et al., 2011). Those with more individual resources 
(namely, situated agency) ${ }^{12}$ are more likely to have a greater capability to make claims on work-family balance, especially when conversion factors are also suitable, such as when work-family balance arrangements or gender equality ideologies are embedded in policy and practice. The level of workplace firm is the place where claims for workfamily balance are made and granted or denied. A workplace organizational culture that reflects sensitivity to employees' work-family balance can be seen as a site for converting policies into work-family balance claims. At the same time, organizational culture can also affect how and to what extent employees face potential penalties and risks to work-family balance claims, including job loss and discriminatory treatment in pay and promotion (Hobson et al., 2011).

Other scholars also recognize that organizational culture has a direct relationship with work-family balance since it can intentionally or unconsciously influence the values, attitudes and activities of employees (Allard et al. 2011). Still, very little is known about the organizational conditions that facilitate work-family balance (Mayo et al., 2011). Like culture within the family, culture within an organization can also be more or less traditional. A traditional organizational culture views work and family as separate spheres with strict temporal and spatial boundaries, where work is done during working hours and personal matters are taken care of during non-working times. Less traditional organizational cultures also exist where more integrative and genderegalitarian opportunities are provided, and where the culture supports parents' attempts to harmonize work and family (Allard et al., 2007). As O'Brien and her co-authors (2007) stress, there is a potential contradiction between evolving conceptions of caring fathering and more traditional notions of employment-driven masculine identity. Holter (2007) found the same using empirical data about European men; i.e., that a more equal gender approach often encounters more traditional organizational gender roles at the company level. It is especially post-Fordist organizations, with their long working hours and high performance pressures, that can reinforce the traditional separation of spheres

\footnotetext{
${ }^{12}$ As mentioned in references to boundary management styles, perceived control is important for positive work-family outcomes and lower job stress. Psychological control acts as a resource that enables individuals to perceive that they have significant resources to handle the demands of jobs (Kossek and Lautsch, 2012). Moreover, Mellner and her colleagues (2014) highlight the individual's capacity for selfregulation as being vital for high boundary control. This reflects capacities such as being able to judge when a work assignment is completed, being able to work independently, organizing work in an efficient manner, and having the capacity to say no and set limits. They find these skills to be the general elements in an individual's capacity to set boundaries, and as such, subsequent boundary control.
} 
and gender roles, as well as traditional masculine cultures in management (GregoryMilner, 2009; Ford - Collinson, 2011). Consequently, there tension may arise between new relational orientations and older organizational structures, often creating high costs for gender equality for men with newer attitudes (Holter, 2007; Gregory-Milner, 2009). The next sub-chapter describes how organizations can convert employees' work-family balance claims into practices - or more precisely, what tools organizations can offer to their employees, and what the norms, informal relations and deeply held convictions are that hinder the success of these work-family policies.

\subsubsection{Organizational Contributions to Work-Family Balance}

An organization can contribute to employees' work-family balance at the policy level in several ways. Wajcman and Edwards (2005) describe four types of direct, formal work-family support: leave provisions (such as parental and family or carer's leave); provision of flexible hours (including part-time work, job-sharing, flexible start and finish times); childcare provision or assistance; and support measures (counselling and referral services). Such policies do not only apply to people with children, since increasing numbers of people are caring for sick or elderly relatives due to the ageing of the population. Tóth (2007) categorizes international organizational initiatives slightly differently to Wajcman and Edwards (2005): she also includes provisions for leave, as well as the provision of flexible hours, but for support measures she describes a broader category which includes relaxation and stress management opportunities (such as fitness centres, sport fields or massage). In addition, her typology includes financial and service in nature provisions (such as coupons, vouchers, financial contributions to studies and training, or free mobile use) as a further category.

As mentioned in Chapter 2.3 about time-based conflict and post-Fordism, flexibility is usually seen as the most important provision among all the work-life balance arrangements. Hochschild (2001) also found in her work that employees primarily take advantage of initiatives which help organize work; namely, those that increase flexibility and work autonomy. Hobson and Fahlén (2009) draw attention to the fact that two meanings of flexibility can be differentiated: employer-driven flexibility and employee-driven flexibility. Employee-driven flexibility is the individuals' capability of organizing their working time and/or rearranging their workday schedules to suit the needs of their families, in contrast to employer-driven flexibility which refers to the employer's needs for greater efficiency (Hobson et al., 2011). The authors add that it 
could be assumed that "those with the greatest flexibility and control over the work situation and pace of work are best able to respond and cope with the needs of family situations. On the other hand, those with the most autonomy often have jobs that place the most demands on them". (Hobson - Fahlén, 2009:223)

As described in the previous chapter about social support (Chapter 2.5) organizational work-family support not only consists of formal provisions, but indirect support as well, such as a work-family climate in which workers feel they do not have to sacrifice effectiveness in their family roles to be able to perform their jobs, or win the support of supervisors and colleagues (Kossek et al., 2011). Several studies highlight the fact that the role of the direct supervisor is often more influential than any formal contributions: Primecz (et al., 2014) showed that direct supervisors and colleagues had the biggest influence over whether employees were able to take advantage of alternative schedules or whether mothers could return to work after maternity leave. An American study (Ladge et al., 2014) made similar discoveries: although male employees had access to (formal) flexible working arrangements and/or other programs, many of them built flexibility into their working schedules through more informal means in which social connections played the main role. For example, support from colleagues and supervisors who were also parents encouraged more involved fathering views to be expressed. Rantanen and her co-authors (2011) suggest that it is particularly important to build work-family balance policies, as well as have supportive supervisors, in order to both promote work-family enhancement and prevent work-family conflict.

A supportive culture also means increasing employees' entitlement. Kossek and coauthors (1999) argue that managers are critical to the success of flexible schedules because they help communicate and implement them and can act as role models. This understanding can be connected to Hobson's (et al., 2011) use of Sen's (2008) capabilities paradigm in the sense that managers' play a role as change agents by pursuing flexible schedules themselves. Managers can help alleviate employee fear by leading by example and making salient to others that it is acceptable to prioritise personal time demands over work time demands, and that high performance at work and home are equally valued. If managers do not use alternative schedules, they send the message to subordinates that the use of flexible options and being career-oriented are mutually exclusive. The authors emphasize how effective the social influence of peers, group norms and departmental productivity issues can be. The more managers have productivity concerns and associate these with flexibility, the less likely they are to have 
flexible schedules. Their findings demonstrate that personal characteristics like gender, and social factors like peer use are strong and consistent predictors of the former or intended future use of flexible schedules.

In addition, work-family balance initiatives within an organization still usually target women as if work-family conflicts only threaten them. Women are more likely to apply for and utilise flexible working practices. Furthermore, it is more likely that a wide range of flexible work options are available in female-dominated organisations than male-dominated workplaces (Thompson, 2010). According to Primecz and her coauthors (2014), this strengthens the belief that women need help and support, which reproduces gender inequality. In addition, organizational work-life balance initiatives can also be considered a tool for exploiting women since these programs socially isolate women and raise expectations about their work and family life. The more support women receive from a company to fulfil their family duties, the less they are treated as fully valuable employees, and also, their housework burden increases. Such initiatives can thus be seen to be supporting the traditional division of labour and hindering the creation of a more equally divided responsibility.

Holter's (2007) findings, however, contrast with these and paint a more positive picture regarding the effects of organizational initiatives. This author claims that women's advancement in organizations has typically been associated with men's advancement, although the link is highly complex. Some companies define gender equality as support for women in ways that have been considered reverse discrimination by many men, and that sometimes women have been gatekeepers for change that also affects men. However, in general, the improved status of women within an organization means greater gender equality for men as well. These changes toward higher equality can be seen as fragmented in a relational and personal sense, rather than collective and organizational. Although some organizations have changed, the study found relative organizational inertia, passivity, and informal reluctance. Men's need for gender equality has become increasingly well recognized, but as a whole, this process is at an early phase.

In context, we should also take into consideration the effect of the economic crisis which started in 2008. It can be seen from the previous chapters that much of the literature (Collinson - Hearn, 1994; Wajcman-Edwards, 2005; Connell, 2005, 2006b) deals with the effect of career individualization and uncertainty on managerial masculine identity. The economic crisis can be considered a source of many 
uncertainties and risks. It can be assumed that the economic crisis has retarded the progress of gender equality and work-family initiatives and has resulted in the maintenance of more traditional forms of masculinity. Insecurity, transformations in and the speeding up of paid work in a globally competitive environment can strengthen assumptions about separate spheres and undermine progress towards the creation of more gender equitable workplaces and force a retreat towards more traditional gender roles (Lewis et al., 2007; Szalma - Takács, 2013). In addition, joblessness generates a sense of risk and insecurity among employees which affects their agency and ability to claim a work-life balance (Hobson el a., 2011). As a more optimistic scenario, it may be hoped that the economic crisis has forced people to find alternative solutions (such as dual-earner households) which may be leading to the creation of more gender equal conditions. Nevertheless, based on empirical findings it appears that this is not the case. In most European countries, the economic crisis has halted the positive trends towards gender egalitarianism and work-life balance support, since in situations of financial uncertainty neither the state nor corporations have focused on reducing work-family conflict or improving women's labour market participation (Szalma, 2014). Primecz and her co-authors (2014) also found that during the financial crisis Hungarian companies had to cut back on work-life balance policies due to the need for cost reductions. According to Szalma's longitudinal study (2014), insecurity in work has resulted in employees having to work even more - on the one hand, to help the company overcome difficulties, and on the other to prove one's value and avoid dismissal from work. In the context of the crisis, flexible working hours and alternative working schedules further increase disorder and unpredictability, increasing workfamily conflict. Work insecurity has affected the genders differently: it has increased work-family conflict in the case of European men, but not women. This can be understood through traditional gender roles and the fact that men have typically used flexibility to show evidence of greater commitment to the company, since they still face the expectation that they should be the family breadwinner. In addition, sectors which were more affected by the crisis (such as heavy industry) were those in which men were overrepresented. Moreover, while before the crisis evidence indicated more workfamily conflict in the Nordic countries than other European countries, this gap narrowed during the crises, perhaps due to a general increase in work-family conflict in Europe. 


\section{FORMER EMPIRICAL FINDINGS}

The chapters so far have described the main theoretical concepts employed in the dissertation and some general trends and policies regarding the relationship between work and family, both from an individual and an organizational perspective. This chapter continues by describing prior international research and the empirical findings connected to work-family conflict in general, and to the three specific types of conflict. The summary of international findings is followed by a brief description of national work-family research embedded in the Hungarian social context.

A search for research into the work-family or work-life interface indicates how rich this topic is with both theoretical and empirical material. The variety of terms and concepts used in work-family literature is also mirrored in the research methods. Consequently, findings are often contradictory due to the diversity of conceptualization and operationalization of the topic. This diversity can be identified even when the search is narrowed to studies which only investigated work-family conflict from among the many work-family interface types. Therefore, the aim of this chapter is to summarize, without claiming to be exhaustive, the main features and general findings of these work-family conflict researches. First of all, two important dimensions will be taken into account before going further into the three conflict types: the gender aspect of work-family conflict, and international comparative studies.

As Emslie and Hunt (2009) conclude, many work-life or work-family studies do not take into consideration the gender aspect of this topic. The majority of research still focuses on how women, particularly in high-status jobs or in dual-career families, are able to harmonize work and family demands (Guest, 2002; Emslie - Hunt, 2009). As already described, despite the changes in the labour market, family structures and gender, the uninterrupted full time model of men is still often taken for granted (Burke, 2000). Consequently, far less research has investigated how men perceive the connection between work and family life. In those cases when research has included men, it is usually in the form of a comparison of the two genders (Martinengo, 2007). Quantitative studies have attempted to control for gender difference by comparing men and women in similar occupations, while relatively few qualitative studies have focused on whether men and women have different perceptions (Emslie - Hunt, 2009). A gender comparison can be fruitful, although it has the disadvantage of putting too much 
emphasis on the differences between men and women, thereby creating dichotomy and opposition. Such an approach can neglect the great variety that may be found within groups; namely, between women and women, and men and men. Consequently the 'within-gender"' perspective is at least as important as the 'between-gender' perspective (Cinamon - Rich, 2002). Even gender comparative research has resulted in mixed and contradictory findings: some studies have found that women report more and different types of conflict between work and family life than men (Emslie - Hunt, 2009). The findings of Emslie and Hunt (2009) based on semi-structured interviews with Scottish men and women in mid-life, for instance, show that although the presence of children in the household was associated with a lack of work-life balance for both men and women, these difficulties lasted longer, and took more complicated forms for women than men. Accordingly, much quantitative research has found that although both men and women face work-to-family conflict more frequently than family-to-work conflict, the latter is more typical for women due to their greater family responsibilities (Greenhaus - Parasuraman, 1999; Hill et al., 2004). In contrast, Hochschild (2001) argues that men and women perceive increasingly similar experiences regarding the harmonization of work and family life. According to this author, both men and women regard home as an additional place besides work, while the workplace is often seen as a haven. Greenhaus and Parasuraman (1999) argue that the studies that find no gender difference in terms of conflict are more difficult to explain. Their first understanding is that it is possible that women self-select into occupations and jobs that are less demanding and which are less likely to generate work-family conflict. They further assume that women have already responded to the conflicting demands of work and family by reducing their involvement in work or by deciding not to get married or have children. These choices might reduce women's perception of work-family conflict to the level experienced by men. Finally, women may be better than men at 'multitasking' or at adopting other coping strategies that reduce work-family conflict to a more manageable level.

Thanks to the rich empirical literature on work-family conflict (including comparative work) we have knowledge of international similarities and variations. According to one quantitative international comparative study (Van der Lippe et al., 2006), employees in post-socialist countries face less work-family conflict than employees in northern- and western-European countries, although the latter place more emphasis on work-life balance. Similarly to this finding, research using the fifth round 
of European Social Survey data (Geszler, 2014) showed that the perceived family-towork conflict of men was higher among Scandinavian countries than in conservative countries like Hungary. Szalma's (2014) results are also based on European Social Survey data and show that the perceived work-family conflict was higher in Nordic societies than in southern European and liberal countries, although this gap had diminished by 2010 due to the influence of the economic crisis. A quantitative analysis of manager fathers in Sweden conducted by Allard and co-authors (2007) confirms these results, showing that conflict can still be high, even in a country with a long policy legacy of promoting gender equality in the workplace.

The reason for this paradox may be that the emancipation which has occurred in western and (especially) northern countries expects both parents to be active participants in the labour force, as well as at home, resulting in conflict between the two spheres. Having a working spouse may negatively impact a husband's work and life satisfaction. Men in dual-career couples experience greater work-family conflict than those in more traditional marriages (Ladge et al., 2014). In the post-socialist countries, however, more conservative expectations tend to support the traditional division of gender roles (Van der Lippe et al., 2006). Also, according to Allard (et al., 2007), those couples who divide domestic labour in traditional ways seem to have less work-family conflict. In addition, work-life balance and gender egalitarian initiatives are less in the public discourse in societies such as those of the post-socialist countries. These might create less attention to the work-family conflict, especially among men. As already discussed, the role of provider can be particularly salient to men's identity as fathers. Fathers who see providing as an act of involvement may not feel that their work and family lives are in conflict (Hill et al., 2004). It seems somewhat contradictory to this observation, however, that according to the findings of an American quantitative study (Ladge et al., 2014), involved fathering is beneficial for organizations due to fathers' higher job satisfaction, commitment to their work and lowered propensity to quit, as well as due to indirect benefits such as improved well-being. According to this, the more time fathers spend with their children on a typical day, the more satisfied they are with their jobs, the less work-family conflict they report to having, and the greater work-family enrichment they experience. Similarly, the above-mentioned empirical results of Allard (et al., 2007) from Sweden show that those fathers who report to experiencing greater gender egalitarianism in their family lives (i.e., who share equal responsibility for children and live in a dual-career family) and have access to flexible 
working arrangements were the least likely to encounter work-family conflict. In summary, a contradiction exists in that although involved fathering has a positive influence on fathers' well-being, and Nordic countries promote it on a social policy level, structural constructs such as assumptions about how work should be carried out, the idea of work and home as separate spheres, the ideal employee model and the need for at-work presence are still strong enough to hinder involved fathering and create conflict for those who would claim for a more balanced life.

According to occupational background, professional and managerial-level men face more work-family conflict than men from other professions (Van der Lippe et al. 2006; Geszler, 2014; Allard et al., 2007; Szalma, 2014). It is a paradox again that managers are the individuals who usually have the best access to flexible working arrangements, yet it is this group that experience more work-family conflict (Allard et al., 2007). Holter's results (2007) involving European employees confirm that new and nonstandard arrangements in organizations could be clashing with the traditional expectations of organizational surroundings, most frequently in central and southern Europe, and for younger generations who are increasingly calling for different models of work (Holter, 2007).

It can be seen that the picture of the managerial father's work-family balance is unclear. Many (often contradictory) factors can influence this, such as commitment towards work, commitment towards family, managerial position, access to flexibility and work autonomy, workload, traditional work expectations like the idea of the ideal employee, family expectations concerning involved fathering, or a mixture of both. The following sub-chapters briefly summarize some general findings regarding the three types of work-family conflict: time-based, behaviour-based and strain-based conflict.

\subsection{Time-Based Conflict}

Based on the differentiation between the two conflict directions of Greenhaus and Beutell (1985) (namely, work-to-family and family-to-work conflict), researches usually also differentiate between work-related and family-related conflict sources. Family-related sources can include marital status (married persons have been found to experience more work-family conflict than unmarried individuals), the presence of children (parents perceive greater work-family conflict than non-parents, especially when the children are young) or family size (large families are more time-demanding than small families). Having a partner and having children (especially) involves major 
responsibility and demands on an individual's time resources, and can therefore contribute to work-family conflict (Greenhaus - Beutell, 1985). Work-related factors first of all include the type of work, number of working hours, frequency of overtime, workload, flexible schedule opportunities and work autonomy. Not surprisingly, a higher workload, the amount and frequency of overtime, the presence and irregularity of shift work and the total number of hours worked and commuted per week are positively correlated with work-family conflict (Greenhaus - Powell, 2003; Burchell et al., 2007; Ladge et al., 2014).

As already mentioned in the previous chapter, the influence of alternative working schedules and flexible working opportunities on time-based work-family conflict is unclear. A U.S. longitudinal dataset, for example, supports the idea of flexibility stigma and shows that men who take a career break, reduce their hours, or are opt of the labour force for family reasons face a drop in their earnings (Williams et al., 2013). Similarly, Norwegian mixed methods findings about professional fathers (Halrynjo - Lyng 2013) show that 'overly' long parental leave leads to the risk that, without sustained visibility and presence, professional men lag behind in comparison to their more motivated colleagues.

Van Echtelt and co-authors (2009) undertook research based on data from the Time Competition Survey which includes 1114 Dutch respondents and their partners from 30 knowledge-based, post-Fordist work organizations in the Netherlands. The authors state that work boosts the male model of work, and thus exacerbates the timegreediness of work. Secondly, household work is time-greedy as well, so employees with substantial household duties (mostly women) are less likely to work overtime. According to these authors, both men and women are equally subject to the time-greedy forces of the post-Fordist workplace, but since women have more difficulty in accommodating these time claims, they are less likely to work in post-Fordist contexts. Although the authors add that new technology and flexibility can improve the work-life balance of employees, since these reforms are introduced in pre-existing organizational cultures based on gendered assumptions about what it takes to be an ideal worker, results confirm the scepticism which exists about flexible working arrangements. As the research suggests, it is not the superficial features of the boundary between work and home but the underlying structures of time competition that must be addressed, otherwise flexibility and the opportunity to work beyond contracted hours make work even more time-greedy. 
A quantitative study about working parents in Sweden (Eek - Axmon, 2013) found that flexible working hours were associated with higher work-related demands, but at the same time, higher work-related control and work engagement, resulting in an 'active' work situation. The authors differentiate an active work situation from a 'strained' work situation where high demand is combined with low control, implying the risk of negative physical and psychological effects. Although the opportunity to work from home was also associated with higher work engagement and higher workrelated control, it caused higher levels of conflict, especially among men. Similarly, Tausig and Fenwick (2001) found based on national survey results from the United States that alternative and flexible schedules did not improve greater work-family balance. Their research found that women reported equal levels of balance to men, but this was achieved by working significantly fewer hours. The use of part-time work as an alternative working schedule, however, created economic costs for them in terms of wages and career advancement. Flexible schedules only lead to higher satisfaction for both men and women when accompanied by a perception of schedule control. Consequently, flexible work only had positive effects in the case that the employee had a high level of work autonomy.

As Hochschild (2001) concluded, flexible working opportunities can even increase the number of hours spent working. Qualitative research about UK managers (Ford - Collinson, 2011) arrived at the same conclusion: that employees who have the greatest degree of control and flexibility over their working hours, rather than seeking to use flexibility to fit in family and recreation, use the time to work longer hours in ways that exclude personal life and leisure time. This does not necessarily happen due to external pressures but rather from internal motivation: international exploratory qualitative research (Lewis et al., 2007) highlights the discourse of personal choice and individual responsibility which was voiced by participants in all the countries investigated regarding the perceived choice of whether to take advantage of flexible working policies. Although some say they are happy with the 'choice' to work long and hard, regardless of culture, many blame themselves for not being able to 'get the balance right' as concerns the blurred boundaries between work and family. Similarly, according to research by Brandth and Kvande (2001) based on three different quantitative surveys with Norwegian fathers, collective and standardised forms of leave such as the father quota have had significant impacts on fathers' involvement in child 
care, but optional, non-standard flexibility and leave solutions have not created mass practice since they have remained at the discretion of the individual.

Technology usage has also great importance in the question of flexibility. The study of Wajcman and co-authors (2008) investigates the effects of phone usage on work-life balance on an Australian representative sample. The authors found that mobile phone was a tool for achieving better balance rather than a work extension device. Survey respondents reported that calls on the mobile were predominantly for social or leisure purposes, for managing home and family, or for other interpersonal contacts, while work-related calls had a low volume (although the last one was more typical for men, than women). Consequently according to the results mobile phone usage did not lead to increased level of negative spillover between work and home.

\subsection{Behaviour-Based Conflict}

As previously explained among the family-related factors, parenthood is found to be a significant source of conflict due to the increased demands on time, attention and energy that child rearing involves (Greenhaus - Beutell, 1985). A U.S study (Chesley, 2011) on stay-at-home husbands based on in-depth interviews with 21 married couples found that at-home fathers come to value their increased involvement in children's care in ways that reduce gender differences in parenting and that have the potential to translate into institutional change, particularly when these individuals re-enter the labour force. Furthermore, at-home father arrangements generally appear to provide increased support for women's employment and promote changes in women's working behaviour. Although the decision to stay at home was in many cases significantly influenced by economic conditions, the shift in family arrangements ultimately promoted a change toward greater gender equality, even in couples that initially held entrenched, gendered beliefs.

The above-mentioned American study of Ladge and co-authors (2014) showed that although father employees expressed a desire for greater balance between their work and family lives after they became fathers, at the same time they described their need to work harder and take their jobs more seriously so that they could provide for their families. Even when men have egalitarian views of parenting and prefer to be more involved with their children, these ideals are often overshadowed by 'pragmatic realism'. Young male professionals in particular focus on practical concerns over idealism when considering their future roles as fathers. Similarly, Halrynjo (2009) 
conducted international, mixed methods research on 102 European men and developed a typology of work and care adaptations based on their prevalence. The author stresses that a care position, even if it is occupied by a man, plays an important role in sustaining career positions since carers support the naturalization of demands for an unencumbered career. The article concludes that even if several work-life adaptations are available to men, they will not necessarily contribute to altering the hierarchical distribution of career and care, privileges and costs in society, since the explicit and implicit expectations of working life still favour the unencumbered worker and maintain the symbolic capital of the work devotion schema. Although men in the sample who worked overtime were dissatisfied with their working hours and expressed their desire to work less, they found that doing so was impossible in their given career circumstances.

Although Nordic countries are considered to show an example of how men's participation in child care and household responsibilities can be encouraged, Swedish results show that even those Swedish fathers who described themselves as involved fathers can be rather classified as child-oriented than as individuals who take responsibility for doing routine chores (Forsberg, 2007). A recent, diary-based time use survey about the involvement of Norwegian fathers (Kitterød - Rønsen, 2013), however, found more positive results about the increases in time that men were spending on both childcare and routine housework as well. According to this research, in modern Norway men and women have on average roughly similar workloads when both paid and unpaid tasks are taken into account. Men also report that work-related requirements interfere with their family life at least as often as women do. It is a question whether more involved fathering practices mean that the double burden of market and domestic work are increasingly being shared by fathers as well. But since responsibilities are shared approximately equally, Kitterød and Rønsen (2013) argue that even mothers seldom bear the double burden in contemporary Norway ${ }^{13}$.

A critical discourse analysis on related articles (Wall - Arnold, 2007) shows that men can be both traditionally masculine, and involved fathers. The two can be combined in ways that do not involve redefining what it means to be a man. The same is

\footnotetext{
${ }^{13}$ However, the authors (Kitterød - Rønsen, 2013) draw attention to the fact that time diaries tend to underrate mothers' total workload, for example, in case of organising and coordinating the family's daily life which managerial practices are rarely captured by time diaries. Moreover, mothers' time use tends to be more fragmented than fathers'. Therefore, mothers may experience more time pressure than fathers, even though they have less total work commitments based on conventional measures.
} 
confirmed in a study by Rudberg and Nielsen (2012) using interviews with three generations of Norwegian men: the young generation of men considers the ideal man to be someone who is able to combine the tough with the soft. However, managing this in practice is not always easy; neither ideals nor identification patterns imply the existence of harmonious, conflict-free solutions. The emotional context for the youngest generation in the research involved mothers' struggle for autonomy and visibility, and fathers making rather insecure adjustments to new cultural gender constructs with wavering emotional investment. As a consequence, the young generation of men identify themselves with nobody. The result is likely to be a compromise between demands for individualization and care. Consequently, having a strong relationship with a child might actually make it possible to combine a desire for both closeness and autonomous creativity.

In addition, class might also matter in terms of how fathers understand their parenthood. In Plantin's (2007) study, Swedish working-class fathers saw fatherhood as a natural part of a planned life course; having children was expected and did not radically change one's life. In contrast, middle-class fathers viewed fatherhood as a rupture that would revolutionize life and identity - once you become a father you will never be the same again. It seems that good, modern fathering is often associated with white middle-class behaviour, while bad fathering is related to poor, immigrant fathers.

As described in the theoretical chapters, the difficulty of harmonizing work and family responsibilities can be even more pronounced in demanding occupations like managerial or professional positions. Based on qualitative in-depth research with UK managers, Ford and Collinson (2011) found empirical evidence of work-family conflict emerging from the expectation that managerial employees should conform to the hegemonic male model of employment which tends to ignore responsibilities outside work. Besides the pressure of an increasing workload, employment is clearly an important part of managers' lives and identities and their accounts are replete with ways in which they use work to reinforce their sense of a 'successful' and 'professional' self. Manager men in the sample expressed a sense of guilt about spending time at home with the family instead of working, and when at work felt guilty about not spending more time with their families. Several managers justified the primacy they give to work with the promise of a better life for their families as the consequence. Others present work as an addiction, without which they would be bereft. Still others see work as an encumbrance and something they would give up if they could, so as to spend more time 
on interests outside work. However, instead of blaming structural factors for the high volume of work and time pressure, managers frequently take personal responsibility for their activities.

\subsection{Strain-Based Conflict}

As described in the theoretical chapter, much research focuses on the relationship between stress and work-family conflict. It has been found that work stressors can produce symptoms of strain, such as tension, anxiety, fatigue, depression, apathy, and irritability (Greenhaus - Beutell, 1985). Thus stress that stems from competing work and family obligations can affect health-related behaviours, even sleep. Interview research with dual-earner couples (Maume et al., 2010) investigated individual sleep patterns in the nexus of gendered work-family responsibilities, finding that among delayed sleepers most men referred to financial and work-related concerns as the reason for their difficulty in getting to sleep, while women lost sleep worrying about interpersonal relations within their families. In addition, men usually enjoyed unbroken sleep after falling asleep, while women's sleep was interrupted due to childcare-related activity. Thus stress from multiple demands increases the risk of fatigue and exhaustion, especially after the arrival of children. A longitudinal quantitative study about Swedish working mothers and fathers (Nordenmark, 2004) shows, however, that a desire to decrease the level of engagement in paid work emerges only among women when high demands from employment are combined with high demands from the domestic sphere. When the combined pressure from work and family gets too high, many women may feel compelled to direct their energy towards the family, while men feel that their main goal should be to maintain their position at work. Similarly, another Swedish study (Eek - Axmon, 2013) found that the subjective health symptoms that employed women report to having are affected by the interaction between working conditions and household duties, while men's health appeared to be more affected by work-related factors.

Regarding the relationship between social support and strain-based work-family conflict, empirical results confirm the assumption that spousal support plays a significant role in how well individuals can manage stress that stems from work and family demands (Grzywacz - Marks, 2000; Greenhaus - Beutell, 1985). Based on observational and diary research on American couples (Neff - Karney, 2005), wives tend to provide better support on days when their husbands experienced greater stress 
and are more likely to increase their workload at home on these days, thereby giving partners relief from the chores at home. However, when wives experienced greater stress, their husbands displayed both supporting and negative behaviour towards them. Greenhaus and Parasuraman (1999) concludes from a review of several empirical pieces of research that husbands receive more instrumental support from their wives than they provide, specifically in terms of housework and child care. As concerns emotional support, however, men and women seem to provide a similar level of support and benefit equally from support at the same time.

In connection to workplace support, Eek and Axmon (2013) found that experiencing the supportive attitudes of colleagues and managers is related to positive outcomes such as lower levels of stress, fatigue, subjective health complaints, and experienced work-family conflict, as well as higher work engagement and general selfrated health. Quantitative research with male MBA graduates in Canada (Burke, 2000) also confirmed that managerial and professional men who reported experiencing higher levels of organizational support also claimed to work fewer hours and fewer extra hours and had less job stress, greater joy at work and greater job and career satisfaction, were less inclined to quit and had higher levels of emotional wellbeing. A U.S. survey with 1000 managers (Kossek et al., 1999) strengthens empirically the assumption that the use of flexible schedules by one's peers can favourably influence the use by individual managers in the same unit. According to a study by Mellner and colleagues (2014) which was based on web-questionnaires conducted with a Swedish telecom company, there was a strong preference for segmentation. Their findings point to the fact that men benefit from a segmentation strategy in terms of both boundary control and work-life balance. In contrast, women face difficulties with segmentation when the working environment 'forces' higher levels of integration and flexibility. According to a survey by Mayo and co-authors (2014) with managers and their spouse in Spain, managerial control neutralized the dysfunctional effects of managerial job demands and strain on work-family balance. Moreover, social support accentuates the influence of job control and helps to buffer the effects of job demands on strain.

\subsection{The Hungarian Context}

Turning from the results of international research to the Hungarian context, it can be stated that, as a post-socialist country, Hungary has already experienced a high level of participation of the female labour force as a consequence of forced 
emancipation. Although gender differences were pronounced, the goal of policies was to integrate women into paid labour rather than exclude them. The state also placed emphasis on motherhood by emphasizing women's responsibilities as mothers and granting them the right to carry out certain care duties. In addition, a well-developed system of child care supported women's full time employment. However, divisions in the family were unequal as household and care tasks remained the responsibility of women, while men were not encouraged either by society or by state policy to take part in care work and housework, resulting in the dominance of the male breadwinnerfemale caregiver model (Nagy, 2010; Kispéter, 2012).

Since the socialist era there has been still no discussion about the need for men to take part in the household and childcare tasks. Despite the significant labour market activity of women and generous state support for parental leave and day-care during socialism $^{14}$ (Hobson et al., 2011), the domain of the home remained strictly women's. Post-socialist countries have in general tried to reintroduce the traditional familization regime and restore the model of the male breadwinner and the related private-public division of gender roles by inducing women to return to the home. Takács (2013:200) describes the fact that women have to face contradictory normative expectations while being dragged back and forth between work and home as a 'historically determined gender yo-yo effect'. Even the participation rates of women in the labour force have somewhat declined compared to the socialist era, and their labour market position has become vulnerable as a result (Fodor - Balogh, 2010). Re-familization policies have strengthened the model of separate gender roles. Even if some policies - such as those relating to parental leave - are coded in generally neutral terms, given the existing division of household labour, the prevailing social norms and the structural conditions on the labour market, they still encourage women to take sole responsibility for the household, while leaving the man to be predominantly defined by his social role and social identity as breadwinner (CROME, 2005; Saxonberg - Sirovatka, 2006; Holter, 2007; Nagy, 2008). It can be seen that the increase in the number of dual-earner household had completely different consequences in western Europe than it did in post-

\footnotetext{
14 "In Hungary 180 days within two years prior to the birth is needed for parental benefits. The national policy provisions on child care leaves allow for and exceptionally long period of 3 years, and incomerelated two-year benefit at 70 percent of income and a low flat-rate benefit that functions as a disincentive for the continuous participation of women in the labour market. The 3 year benefit reinforces gender role expectations, as it assumes that mothers will be taking the leave, reproducing the gender divide in care." (Hobson et al., 2011:177) Returning to the labour market after a long period is quite problematic, especially for mothers with a lower level of education. The actual rate of return to work is less than $45 \%$ (Hobson et al, 2011).
} 
socialist central and eastern-European countries. In western countries, the entry of women to the labour market happened in tandem with gender emancipation processes, while in eastern counties, since female participation in the labour market was primarily economically driven, traditional views about the division of household labour were not influenced (Van der Lippe et al, 2006). The attitude of western Europeans towards gender roles has generally become more liberal over the last 25 years, while the views of Hungarians have not demonstrated a similar tendency. In addition, in Hungary strong opposition to the concept of feminism has emerged, coinciding with a lack of political action and organization for women. Accordingly, attitudes towards gender roles have more or less remained frozen at the point they were when state socialism collapsed (Fodor - Balogh, 2010).

Recent empirical findings confirm the unequal division of labour in Hungary, as well as the traditional attitudes of society. Even if a couple follows Western patterns of sharing housework responsibilities before they have a child, after the birth of the baby the traditional division of labour tends to re-emerge (Takács, 2013). In representative surveys, around three-quarters of respondents usually state their support for this division of labour in which the father is the economic provider of the family, while the mother takes care of the household and the children (Takács, 2008; Pongrácz, 2001; Nagy, 2008). In 2000 an international comparative study showed Hungary to have the most traditional attitudes among participating countries. $56 \%$ of women and $66 \%$ of men agreed with the traditional model of the division of labour, twice as many as in Germany or Austria. In 2009, however, 54\% of women and 55\% of men agreed with the statement, indicating a significant change in the opinions of men (Pongrácz - Molnár, 2011). Slow changes are also being recorded for other issues: according to time-diary research (Harcsa, 2014) time spent on childrearing has doubled in the past 25 years among Hungarian couples. The father's time spent with children has significantly increased, although, as expected, there is still a huge gap between the genders. Similarly, using data from time-diaries Sebök (2015) found that the time spent on paid work and leisure has decreased, while the amount of hours devoted to child care increased between 1999-2000 and 2009-2010 in the Hungarian population. Men's contribution to house chores has increased which means that women have more time for child care alongside household duties. According to Sebők (2015), it is difficult to tell whether men's increasing contribution will be long-lasting or short-term. She suggests that macroeconomic processes, such as the economic crisis and its individual 
consequences such as increases in unemployment, played a more significant role in the change of how men use their time. Takács (2013) draws attention to the fact that among Hungarian parents, quality parenting (namely, being a good enough parent who spends 'quality time' with a child) is becoming an important element of being a father or mother. Pongrácz and Molnár (2011) highlight the fact that Hungarian society cannot be categorized as either completely 'traditional' or completely 'modern' but may be located between the two poles, with mixed and ambivalent elements. Between 2000 and 2009 there was a remarkable change and fewer people can be now defined as traditional, although this does not represent a clear shift towards "modern" attitudes, but rather that respondents firstly identify with a 'mixed' category.

Regarding the evaluation of the role of Hungarian fathers, Spéder (2011) undertook quantitative analysis based on the 2009 Family Values data from the Hungarian Central Statistical Office. This author wanted to know what public opinion in Hungary expects from men: that they should be the income provider for the family ('the breadwinner father') or that they should be more active in family and care duties ('the egalitarian father'). Similarly to the findings of Pongrácz and Molnár (2011), the findings indicate that a clearly traditional nor clearly modern attitude exists: $94 \%$ of the Hungarian respondents, both men and women, agreed that the most important task of a man is to provide financial security for the family. Meanwhile, $89 \%$ also agreed that fatherhood and taking care of a child is one of the most beautiful tasks in a man's life. In accordance, $82 \%$ did not find it ridiculous if a man changed his child's diaper, although this was one of the tasks considered the most feminine. Spéder (2011) also composed a variable to measure which ideals about men the public relate to: $24.5 \%$ of respondents expected the man to be the breadwinner, without needing to sacrifice their time working on care tasks, while $18.5 \%$ identified with a family-centric man, ready to cut back on work in favour of the family. The majority (48\%), however, raise contradictory expectations towards men by desiring them to be the family breadwinners but also expecting them to prioritize family over work. Regarding some concrete tasks, the respondents found that providing security was the most important task of a father (97\%), while $94 \%$ thought that a father should show his love and emotions towards the child. Surprisingly, only one third of respondents said that undertaking routine tasks (feeding, clothing, changing diapers, etc.) was very important. This means that although modern and family-centric elements of fatherhood are alive in Hungarian public opinion - like the importance of feelings and love - the traditional understanding of fatherhood 
is deeply rooted in Hungarian society, since routine and everyday care tasks are neglected. Spéder also examined comparative data from the Generation and Gender Survey and found that respondents did not clearly differentiate along the gender variable according to whether they thought having a child is a necessary component of individual fulfilment, but they did consider parenthood to be important per se: in Hungary $85 \%$ think that a woman has to have a child to be happy, while $75 \%$ believe the same about men. In Germany, however, only 36\% of respondents find that being a parent is necessary for a woman to have a fulfilled life, while for men the figure is $30 \%$. Consequently, this indicates that to what extent countries think parenthood is a determinant of a happy life is of greater relevance than the gender split (Spéder, 2011).

Investigation of the organizational context shows that while in northern and western European countries democratic traditions are stronger, with more labour unions and labour laws protecting the rights of employees, in eastern and southern European countries employees are more vulnerable and dependant: fewer regulations protect them, the supervisor-subordinate relation is more hierarchical, employees feel that their jobs are less secure and the level of distrust is higher. Consequently, the lack of a democratic structure enhances job stress and job vulnerability (Utasi, 2011). Hobson and her co-authors (2011) also conclude that in eastern European countries employees experience a higher degree of economic uncertainty. As for the gender context of organization, it can be said that the Hungarian organizational and management sphere is characterized by strong gender segregation, both horizontally and vertically. Especially upper management is dominated by men, while women usually are responsible for feminized fields, where they manage mostly female employees (Nagy, 2007).

As for work-family policies in Hungarian workplaces, managerial control is closely intertwined with formal and informal work-family practices (Kispéter, 2012). According to Géring (2014), who undertook content analysis of corporate websites to see what medium-size and big companies in Hungary communicate about corporate social responsibility, a maximum of $5 \%$ of the 146 companies under analysis mentioned flexible working arrangements, part-time, maternity programs or family friendliness among their corporate social responsibility contributions. In addition to these initiatives, alternative opportunities were available in companies which were under foreign ownership and had adapted to international norms. The author draws attention to the fact this does not necessary mean there is a near-complete lack of such arrangements, but it is clear that support for work-life balance is not an identity-forming feature of 
Hungarian companies, since they do not find it important to mention this on their websites. Another piece of research (Hobson et al., 2011) shows that the opportunity to work fewer hours is seen by employees as a sign of family-friendly workplace, although the Hungarian labour market is characterized by a very low level of part-time employment.

\subsection{Hungarian Work-Family Research}

Hungarian scholars have not yet studied in detail the topic work-life or workfamily balance, although in the last few years an increasing amount of work has emerged. Here I summarize in brief the main findings of the research which has included men in the analysis, in addition to that already described so far (Géring, 2014; Szalma, 2014; Takács, 2013; Geszler 2014; Spéder, 2011; Sebök, 2015). During the literature review I first met with the work of Beáta Nagy (2008) who is among those researchers that describe the Hungarian work-life balance situation using a gender perspective. In addition, this work also employs a non-typical perspective by analysing men's attitudes and strategies. Nagy investigated companies which won the "Family Friendly Workplace Award" and analysed whether the male leaders of these companies live a family-friendly life as well. They saw harmonizing family and work expectations as requiring both individual and macro responsibility. Although the respondents sometimes avoided giving a direct answer to the question who was responsible for harmonizing work and family, it seemed that many of them favoured the traditional method of dividing work, and saw work-life balance as a women's issue. The same occurred when talking about the users of work-life balance initiatives. Respondents considered the work-life balance to be primarily relevant to women due to their roles as mothers, which they treated as the primary duty of women. Many of the interviewees spoke out against the 'reversal of gender roles' and only a minority supported the more gender-equal division of labour. Differences in opinion could not be correlated to age; even young leaders represented a conservative approach. Interviewees refuted the idea that the traditional division of labour would reproduce gender inequality, and treated the question as a completely separate concept, denying its existence within the company's operations.

A paradox appeared in that although these interviewees were employed at family friendly companies, in most cases they could not maintain balance in their own lives. They found the source of conflict to be a lack of time and the special characteristics of 
having a leadership position. Moreover, they experienced the disadvantages of workfamily conflict primarily in connection with their children; namely, that they could not spend enough time with them. That little time was seen, however, as 'quality time'. Although their wives created the conditions for men to succeed, the only remaining task of the interviewees was to enjoy time with their children. In almost every case, the existence of a 'two-person-career' was observed; namely, that the interviewee was being supported in their career by a wife. Husbands usually did not see this as the wife's sacrifice, but as an opportunity for her to be a full-time mother - although it was rarely the case that wives were "only" full-time mothers, since they often had part-time jobs or helped in a family business. Finally, another paradox was that these interviewees agreed that although leaders can be role-models for promoting work-life balance among their employees, in practice they mostly fit their family responsibilities into their career schedules and used family resources to achieve their career goals.

Another important work-life balance-related piece of work with an organizational perspective on Hungarian leaders is Herta Tóth's (2007) case study at Unilever Hungary. This researcher conducted twenty-six interviews with both female and male leaders in 2002. She also undertook four interviews with human resource managers to map organizational policy in order to investigate both individual and organizational levels. Her results are similar to those of Nagy (2008); namely, that even if companies are putting some effort into offering family friendly or work-life balance initiatives, employees still face significant conflict when it comes to harmonizing their work and family lives. The main focus of her work is the blurring borders between work and family life, and the phenomenon that work is a component of self-realization. This finding is quite similar to the conclusion of Hochschild's (2001) previously mentioned work, The Time Bind, which states that work can become a quiet shelter for employees. Leaders of the organization had the opportunity to manage their working life in a flexible way, and which resulted in great variety of work scheduling based on individual preferences, although it often led to even more hours of work. This finding is also very similar to what Hochschild (2001) concludes, although employees commonly used working time and organizational resources (such as company cars, unrestricted company mobile phones or the wide range of Unilever products) for private matters. Through the tight cooperation and organizational programs of teams (company dinners, training events, teambuilding, corporate celebrations, etc.), employees often became friends as well, thus turning the working environment into a communal scene. The main 
message is that a big company in a post-Fordist time regime can provide a complex package of support to employees and be more to employees than just a workplace: it may be a supporting environment suitable for relaxing, friendships and social programs and provide employees with numerous resources they can use in their private lives.

Employees with families faced more stress in harmonizing work and family life and considered their partners and children to be a great source of motivation for erecting borders between the two life spheres. Accordingly, they experienced the constant fear of not being able to participate in planned family programs because of the need for unscheduled work. Another attempt to divide work from family was that Unilever employees did not usually like to work from home since they found this disadvantageous to both work and family.

Surprisingly, working time did not explain role conflict, since the members of the board worked longer hours on average yet perceived that they lived a more balanced life. Tóth (2007) explains this by pointing to their greater work autonomy and very conscious problem-solving strategies. This finding accords with the conclusions of Tausig and Fenwick (2001), who state that it is not flexibility itself that helps with achieving greater work-life balance, but control and autonomy over one's schedule. Very similar to Nagy's (2008) findings, male leaders saw themselves as family breadwinners, and although they were aware of the unequal division of household labour, they seemed to be satisfied with their work-life balance and did not intend to change it. They were not considering using any flexible or work-life initiatives, although they found part-time work to be a good solution for their wives. Every male interviewee was able to rely on the support of their wife. The complete dominance of work in their lives cannot be understood as a lack of conflict, but as a simplification of roles

Another important finding of this work is that interviewees (mostly the men) saw sustaining a work-life balance to be the responsibility of the individual, and could not envisage what a company or a state could do to improve it. This is good empirical proof of the claim that was made in the sub-chapter about flexibility and post-Fordism (Chapter 2.3): namely, that while work-life balance remains a problem for the individual, and the responsibility of corporations is hidden, it is very unlikely that changes in the current situation will occur.

These observations can be connected to the next important piece of research about the Hungarian work-life balance situation: a book chapter written by Hobson, 
Fahlén and Takács (2011) about agency and capabilities regarding work-life balance using a comparison of two different institutional contexts, Hungary and Sweden. Based on 100 qualitative interviews per country, the authors focus on examining three core policy areas that may support work-life balance: flexibility, the right to reduce hours, and parental leave. The researchers found that only women were taking advantage of parental leave in the Hungarian sample, reflecting the weak ability of men to secure and use parental rights. Clearly, the previously mentioned economic reasons primarily explain this situation, but both men and women interpreted the organization of care and the gender division in parental leave as natural, by referring to, for example, breastfeeding. The idea of men taking parental leave was unimaginable in the light of normative gendered expectations at the workplace. Although men who work in the private sector were more satisfied with their flexibility than men who worked in the public sector, they still emphasized that they did not want to turn down extra work regardless of whether they had the option to refuse it, thereby demonstrating a low sense of entitlement towards flexibility rights. The primary concern was financial: namely, that by working more they could earn more money. Hobson and her co-authors (2011) also found that men's work-life balance claims at workplace entailed greater risk than for women, who can expect more tolerance towards their desire to prioritize family needs. This finding fits with the formerly mentioned thoughts of Halrynjo and Lyng (2013) about how men are thought to be irreplaceable at work. In conclusion, there is only weak potential for maintaining a work-life balance among Hungarian men and women, who have (or perceive that they have) limited entitlement to take advantage of family-friendly initiatives. The main reasons for these agency inequalities are the structural features of the economy and labour markets, including the prevalence of insecure employment, long working time regimes, and low wages that force mothers and fathers to take on extra jobs. Without the perception that one is entitled to rights, and the agency to take advantage of them gendered norms are not challenged and thus remain deeply imprinted.

Primecz and her co-authors (2014) analysed employee-friendly policies and their effectiveness from a critical viewpoint. Their sample consisted of companies who gave awards for family friendliness or best workplace. These authors used HR, top leader and special employee (returning mother, employee teleworking etc.) interviews as well as focus groups interviews with office managers and employees, document analysis and a motivation survey. The results draw attention to the advantages and disadvantages of 
flexibility. Flexibility was usually the only way in which mothers with small children could come back to work, although they experienced higher level of stress due to the constant feeling of lagging behind, and of not being present while their colleagues were progressing with work. Fathers with small children, however, did not form groups with similar support needs at any company. There were nevertheless examples of individual strategies, such as not organizing meeting during morning hours when fathers were taking children to kindergarten. Although the support of fathers could not be identified on an organizational level, fathers also faced work-family conflict. The source of conflict was time-based, and usually involved time and task segmentation as a solution. Accordingly, fathers were typically responsible for more predictable tasks which could be matched to their working schedules, while mothers dealt with unpredicted situations (for instance, the sickness of a child). Fathers, just as in Nagy's (2008) study, showed little interest in changing their work praxis in order to spend more time with their families. In conclusion, the power asymmetry between male and female employees acted to reproduce pre-existing form of inequality.

Primecz and her co-authors (2014) also emphasize cases in which employees had to take care of an elderly or sick relative. For these issues the companies in the research did not have a unified strategy, but on an individual, ad hoc level they were supportive of their employees. However, companies were not as supportive when it came to employee illness, although it is hard to differentiate between the influence of individual responsibility and company pressure on employees in these cases. There was also a big difference regarding the position of employees: those in higher positions had somewhat more opportunity to maintain their own work-life balance - for example, to ask for more days off than their colleagues in lower level positions. The authors emphasize these power-related asymmetries, concluding that only certain groups benefit from employee-friendly policies which thus do not achieve their original goals of strengthening commitment and trust.

Regarding the consequences of work-family interface, Utasi (2011) investigates negative and positive stress which stems from the labour market and work on women and men. She argues that only few studies have focused on the contradictory expectations men face due to the process of emancipation: men who were previously socialized according to traditional values are both expected to be the breadwinners in the family, as well as to take part in household and care tasks as modern fathers and partners. Utasi carried out analysis based on data collected during the fifth round of the 
European Social Survey, comparing 24 countries, including respondents living in dualearner couples. Hungary is among those countries where the highest level of negative stress stemming from workplace control and supervision is perceived, and individual work autonomy and the level of trust is also low. The number of working hours is also higher than other European countries. Due to the long working hours and the low level of technical-civilization both total workload and apathy are higher, and at the same time there are a lack of creative activities which could compensate for negative stress. Instead, employees choose forms of passive relaxation such as watching television. Negative work-to-family spillover is also the highest in eastern European countries: employees are worried when they have unfinished work and bring work stress home. Men are significantly more stressed about work outside of working hours than women in every country, although in terms of general work stress women face more problems due to the second shift. Work stress enhances conflict between couples, usually about the division of housework and the frequency of overtime. Both working men and women suffer from an accumulation of stress about ambivalent value preferences which they cannot fully satisfy.

Blaskó (2006) undertook analysis on the 2002 ISSP database with a focus on family issues. In 2002, Hungarian men faced more work-to-family than family-to-work conflict. 59\% experienced time squeeze and $68 \%$ stress due to work, while these numbers are $52 \%$ and $40 \%$ in case of family-to-work conflict. Although men take part much less often in house chores than women, they report to facing a lack of time for accomplishing such tasks, most probably because of their workloads. The fact is, however, that despite of the time squeeze at home, they rarely feel that their family responsibilities affect their performance at work, which shows that household tasks are still seen as female responsibilities. Men spend more hours working than women: according to this database the average number of hours worked per week is 45 for men and 38 for women. The number of working hours clearly has a significant effect: $72 \%$ of men who work 40 hours per week (on average) feel that they are too tired to do housework after work. Although fathers are not more active with household tasks than non-fathers, they complain more about exhaustion and time squeeze due to their work.

Last but not least, Judit Takács (2015) recently undertook qualitative research on the topic of active fatherhood. Sampling was based on self-selection so that men who consider themselves to be actively caring fathers and who have a child younger than 7 years old were recruited. As a result, 55 in-depth interviews were conducted that 
included 8 fathers who stayed on parental leave for longer than 6 months with their child. Although the majority of fathers expressed their desire to spend more time with their children, the time constraints of work did not allow this. The self-employed, entrepreneurs and teleworking fathers had the same experience. Rational, economicbased explanations dominate in understanding the overwhelming role of work, especially among those with big families: although the partners had similar levels of education, they earned less than their husbands on average, a fact which legitimized the fathers' presence on the labour market. Above all, presence in itself was mentioned as a core element of an actively caring fatherhood. The men in the research were critical towards their own father's parenting: their presence was precisely the thing which they now missed the most. Compared to their own fathers' behaviour, they regarded their own parenting as more caring and emotional, emphasizing the importance of quality parenting. Moreover, they believed that they shared in responsibilities more equally with their partners than their own fathers had. In summary, the following factors determined the fathering practises: social expectations and norms, a lack of father quota-type incentives, family size, self-employment, teleworking, the labour market situation of mothers, and post-materialist aims. The last item refers to the need to "slow down" and to prioritise being part of a child's life more than chasing after success at work after reaching a certain satisfying point in a career.

Based on these national work-family studies, it can be concluded that Hungarian men and women usually act according to traditional gender and work expectations. This can be observed in the dominance of work in men's lives, the myth of the ideal employee within organizations and men's lack of feelings of being entitled to claim a work-family balance. The work-family balance is still usually seen as an issue for women; accordingly, men rarely consider themselves users of family-friendly opportunities, even if they also face work-family conflicts. The aforementioned studies primarily focus on time-based and strain-based conflict, and refer to time squeeze, stress or flexibility.

The next chapter summarizes the research questions that are formulated based on the literature and empirical findings described so far. This is followed by a brief introduction of the case study and qualitative content analysis methods, and a description of the sampling process used to collect data. 


\section{RESEARCH QUESTIONS, SAMPLE AND METHOD}

\subsection{Research Questions}

The aim of this work is to analyse the conflicts that emerge from work commitments and family demands relating to Hungarian manager fathers. As the empirical findings have previously described, men who display traditional gender attitudes, and, in general, couples with a more traditional gender division between paid and unpaid labour tend to face less work-family conflict (Van der Lippe et al., 2006; Ladge et al., 2014; Allard et al., 2007). Consequently, it can be assumed that no conflict or only a low level of conflict will be perceived by the analysed Hungarian manager fathers. I expect, however, that due to the complex mechanisms behind work-family conflict, certain manager fathers might be affected by time-, behaviour-, or strainrelated problems. The three main research questions focus on interrelated issues of work-family conflict. The first research question concerns the general perception of work-family conflict, and has a focus on different aspects of the phenomenon:

1. How do manager fathers perceive and experience the conflicts between their work and family life?

- What are the sources of these conflicts?

- What are the consequences?

- What are the strategies they use to balance work and family life?

To exceed analysis at the individual level, the relation between managers and companies will be analysed as well. Since the company under investigation is in Scandinavian ownership, it can be assumed that more advanced family friendly and work-life balance arrangements are being offered to employees so they can harmonize their work and family demands. Therefore these initiatives will be investigated with a special focus on flexible working options and their efficiency. Based on the capabilities paradigm (Hobson et al., 2011; Sen, 2008), the aim is to specify those formal and informal constraints, norms, and resources that influence managers' ability to claim a work-family balance. Consequently, the second research question focuses on the relationship between the individual and organizational level in the framework of agency and capabilities: 
2. How does the organization influence manager fathers' perceptions about work-family conflicts?

- To what extent is flexibility stigma and a compulsion for visibility present?

- How entitled are manager fathers to make claims for an appropriate work-family balance, and what are their agency and capability options within the company?

Finally, it can be assumed that the concept of ideal employee and involved fatherhood might create behaviour-based conflict in the lives of manager fathers. The role of Hungarian fathers in general was found (Takács, 2008; Pongrácz, 2001; Harcsa, 2014) to be substantially different from the involved father ideal experienced in Nordic countries, since, for instance, their assistance through parental leave is remarkably low (Hobson et al., 2011). There have been, however, slow changes in fathers' attitudes and time spent with children (Pongrácz - Molnár, 2011; Harcsa, 2014) which together with the existing expectations about the breadwinner role might be creating dual pressures on Hungarian fathers, like those Spéder (2011) describes. Moreover, the breadwinner role cannot be simply associated with the economic needs of the family, but as the literature on managerial masculinity states, managerial positions are also associated with social and symbolic power that binds the masculine identity to business (Bowman, 2007). Therefore the third research question focuses on the different interpretations of masculinities and their influence on manager fathers' work-family balance:

3. To what extent do the discourses on involved fathering and managerial masculinity act as ideals to men, and how do these ideals affect workfamily conflict?

\subsection{Sample}

A large-sized service sector company in Scandinavian ownership was chosen for analysis. The Scandinavian background has already been explained, and the reasons for investigating a large, service sector company are the following: 1) the existence of a post-Fordist time regime (resulting in the blurring of borders between work and home) is more typical of multinational, knowledge based companies (Kvande 2009; Lewis et al., 2007; Allard et al., 2007); 2) these high-commitment corporations are more often regarded as greedy organizations, which might be creating work-family conflict 
(Burchielli et al., 2008); 3) there is a greater chance that formal work-family and family friendly initiatives will exist at large-sized, multinational companies (Van Echtelt et al., 2009); 4) it is also relevant that in Hungary management is dominated by men (Nagy, 2007), therefore presumably even if the schedules of the potential interviewees are tight and the chance of refusing to participate in the research is high, it will not be a problem identifying other interviewees for analysis. To sum up, the phenomena I would like to analyse such as blurring borders, work-life balance and family friendly initiatives, flexibility stigma and visibility are more likely to be found in this environment.

Within this company the sample consists of managers from group manager level to $\mathrm{C}$ level positions (namely, lower level managers to top-level managers), who are already fathers. Since this is a case study, in order to gain a more complete picture, female managers are also included in the sample as a control group using the same selection parameters (lower level management to $\mathrm{C}$ level management, already mothers). Only Hungarian managers were interviewed, so excluded from the sample are those few foreign managers who were working in the Hungarian subsidiary. Managers from abroad might have been differently socialized and have a different cultural background and therefore might perceive the work-family balance issue differently to Hungarian managers. The total sampling approach was used; namely, access to all potential interviewees who met the sampling parameters. The reasons for choosing managers as the sample population are the following: 1) work-family and family friendly arrangements are generally more available to individuals in this position (Primecz et al., 2014); 2) managers have been found to face a higher level of workfamily conflict (Allard et al, 2007; Geszler, 2014); 3) managers are in a position to promote or hinder work-family programs, and can act as role models for other employees (Allard et al., 2007; Holter, 2007); 4) and the discrepancy between the ideal employee and involved fatherhood may be the greatest in this position (Greenhaus Beutell, 1985).

\subsection{Case Study Method and Qualitative Content Analysis}

I have chosen to use a case study method, expecting that the carefully selected context might be the best to observe the above-mentioned mechanisms. Case studies can serve several purposes, such as theory building, exploring, theory testing or confirming findings from other studies, although they are usually believed to serve for hypotheses generation at the early stages of research (Bryman, 1989; Flybjerg, 2006). In this 
dissertation the primary goal is to evaluate and potentially confirm the findings of other studies since I intend to analyse how the mechanisms described in the literature review are valid in the case of the presently described sample. Accordingly, the study is similar to the 'explanatory type' described in Yin's (2003) categorization of case studies. Besides the exploratory and descriptive type of research, explanatory research analyses the presumed linkages in real-life interventions that are too complex for survey or experimental strategies. A case study can include various methods, such as interviews, focus groups, document analysis or participatory observation. This research involves an interview-based case study. According to Bryman (1989), in this type of case study the chief emphasis is on undertaking unstructured or semi-structured interviews in 1-5 organizations. Observation may occur, but in non-participant way with the researcher being very much on the periphery of interaction, and is undertaken in a somewhat unstrategic manner in the periods between interviews or at meal-times.

Case study method is often criticized since it is considered that it is not possible to generalize the results of research based only on one or two cases. As Bryman (1989) argues, however, the aim of case studies is not to draw conclusions from a sample to a population, but to reveal patterns and linkages of theoretical importance. According to Flybjerg (2006), formal generalization is overvalued as a source of scientific development, whereas 'the force of example' is underestimated. It is often more important to clarify the underlying causes behind a given problem and its consequences than to describe how frequently they occur. In addition, case studies have a strong emphasis on context and can show the interconnections embedded in an organizational background (Bryman, 1989). This kind of practical, context-dependent knowledge can be as valuable as general, theoretical knowledge. When choosing a case, one can use an information-oriented selection process (instead of the random sampling used in quantitative research), which is designed to maximize the utility of information from a small sample or even a single case (Flybjerg, 2006). Mayring (2014) argues that qualitative approaches are in general subject-centred and close to everyday life, therefore the theory-driven research process in particular provides adequate construct validity. Objectivity in the sense of the independence of research findings and researcher is not possible, although on the other hand the researcher may be more reflexive and conscious about the interactions which occur between researcher and the subject of study. 
Managerial interviews are semi-structured interviews that are analysed with the use of NVivo10 software. The interview analysis was undertaken according to theoryoriented qualitative content analysis (Mayring, 2014) which offers a system of general principles about the subject that can be examined, and is also regarded as the cumulative experience of others in the same field. As Mayring (2014) states, there are various forms of qualitative content analysis. This author differentiates four main types with subcategories: reduction, explication, structuring and mixed forms. He highlights the fact that qualitative content analysis does not need to be limited to only a single form, but several procedures can be combined, depending on the aim of the research. The research described in this thesis is a mixed, alternative form of the structuring type of qualitative content analysis. Structuring is a deductive category assignment that is designed to establish a system of categorization before coding of the text is undertaken using theory, former studies and research findings. The goal is to extract a certain structure from the material under study by assigning textual components systematically to categories. The reason that this research cannot be labelled as solely structuring is that inductive category assignment was also used, besides the deductive approach. This means that most of the coding categories were pre-defined based on theories and previous research, just as occurs with the structuring style; on the other hand, some additional categories were also established based on the text through an inductive process. Through this combination the advantages of both theoretical considerations and a material-driven approach are obtained. This mixed version of structuring is labelled content structuring or theme analysis by Mayring (2014), and describes a process wherein the first step of category assignment is deductive, followed by inductive category formation as a second step if needed.

Content structuring involved the following steps: the semi-structured interviews were recorded on tape and then transcribed in typed form. The interview transcriptions are extracted transcripts due to the need to save time. An extracted transcript is also a form of word-by-word documentation, just like a full transcript, but 'off-topic' comments or narratives which were not relevant to the research were omitted. Based on Mayring's (2014) typology of transcription systems, this is called 'selective protocol', and involves the researcher defining only those parts of the audio record that are relevant to the research question. The transcripts were input into to NVivo software along with the contents of the Excel table which included the demographic data about respondents, although the interviewees were rather homogenous based on their 
demographic background. As mentioned previously, the creation of categories happened both in a deductive and inductive way: most of the categories were created based on the literature and primary research, such as the three types of parenthood involvement in Lamb's (et al., 1987) typology, while other categories were formulated after reading responses to questions asked in the interview (such as in the case of the company's work-life balance arrangements which were commented on by respondents). The deductive category assignment contains mostly nominal category systems (a list of independent categories) and one ordinal category system (graduation of the structuring dimension). Categories ('nodes' in NVivo) constitute a tree structure, having narrower categories ('child nodes') embedded in broader categories ('parent nodes'). Through this approach the advantages of both broader, more generalized categories and narrower, detailed categories can be used and seen in a structured way. The coding units were sentences or mostly short paragraphs of interview transcripts because it was necessary to understand the context in which the words were spoken. After coding the first 10 interviews, the categories and coding guideline were revised before the final work-through of material. The final step involved analysis of these categories by summarizing the content, checking category frequencies and interpreting contingencies. 


\section{RESEARCH BACKGROUND}

\subsection{Access to the Field}

First, the CEO of the company was approached with the idea of the research. He reacted positively and showed an interest in the topic of work-family balance and delegated the task to the Human Resources Department, where, after contacting the leader, I was directed to one of his colleagues. This assistant at the HR Department was my contact for all the administrative processes undertaken during the research. Although she was very helpful, she had to be reminded several times about requests I had made, and as a result, the administrative process went much more slowly than expected. This can be clearly seen from the fact that although I first contacted the assistant in November 2014 after receiving the permission from the CEO, I only received access to the building and the corporate network at the beginning of March 2015. The HR Department had a discussion about the most suitable method of implementation and decided that, instead of having the assistants of managers arrange interview dates after consulting me, it would be easier if they gave me access to the corporate e-mail system through which I could contact the potential interviewees directly. Looking back, this was truly the easiest solution since I was able to arrange and modify the dates and conditions of interviews directly with interviewees without any middlemen, which speeded up the process. Therefore, at the very beginning of March I finally got a company account and became an official external employee of the HR Department. After signing the requisite confidentiality agreements, I received a company phone, laptop and remote access to the Outlook e-mail system; consequently, I was able access e-mails even from home. This did not mean, however, that I had access other parts of the company's network system. When I needed a document I had to ask it from my contact. The next step was to formulate a letter to potential interviewees about the research request. This template letter was reviewed several times by the HR Department before it was finally accepted. After this step, the HR Department assistant compiled and gave me a list of potential interviewees based on my selection criteria. This table contained the name, age, position, department, number and name of children, e-mail address and phone number of the individuals. With this table and access I finally had all the tools necessary to conduct the interviews. After this point I was left 
unsupervised and could freely move within the company and send e-mails without permission.

After I obtained access my sampling rule was the following: I chose every manager of group manager level and above who had at least one child of 10 years old or younger. E-mails were sent to all those managers who met these criteria, and I started to check their calendars one by one to find a free time for the proposed interviews. This part of the research involved plenty of organizational tasks and I had to adapt to the schedules of every interviewee, which was not always easy due to their busy calendars. This was especially difficult in the case of managers in the highest positions when free time could only be found 2-3 weeks in advance. Besides arranging a date, a free meeting room also had to be found and booked in advance. Thus the meeting request sent to interviewees contained a very brief description of the interview request, a potential date for the interview and details of the place. To my pleasant surprise I quickly received e-mails from many interviewees saying that would willingly participate in the research. Others simply accepted my meeting request in Outlook, or suggested an alternative date for the interview, but all in all the response rate was high.

Fieldwork lasted from 3rd of March 2015 until the 13th of April 2015. During this period interviews with 43 men were conducted out of a potential total of 50, and 15 female interviews out of 28 (i.e. a total of 58 interviews with managers were conducted). In the case of men, the main reason for refusing an interview was that the floor managers were working abroad or in another part of the country. 4 potential male interviewees said no to the interview, providing this reason. One male manager did not reply to my e-mails after two attempts. Two potential male interviewees were helpful at the beginning but after unsuccessfully reorganizing the date of the interview several times due to their busy schedules they kindly asked me to cancel the search for an alternative appointment. Consequently, the response rate was $86 \%$ for men. For women, 7 potential interviewees did not have an email address available in the Outlook system. Since, according my list they had very young children, it may be assumed that they were on maternity leave at that time which is why the system did not allow me to contact them. Although two other female managers had an available e-mail address, they were also on maternity leave and therefore they rejected my request. One female interviewee was working as a floor manager in another part of the country. Only one potential female interviewee did not want to be interviewed due to pressing issues in her private life. One other manager mother who was working in another office not far away 
refused to be interviewed despite me offering to visit her office. Deducting from the total those 7 interviewees who could not be contacted, the response rate among women was $71 \%$. Although a high refusal rate for professional and managerial interviews is often expected due to the busy schedules of managers, my experience was very positive. And, as already mentioned, despite all the organizational tasks this was still the easiest and least circumstantial method for arranging the interviews.

In addition to the 58 managerial interviews I had three informal talks as well: one with the HR assistant who was my contact and who gave me some additional information about the work-family balance policy at the company; one with another HR assistant who gave me a brief presentation about the Employee Engagement Survey conducted with company employees in 2014; and one with a young female manager who took some time to talk with me about the company during a coffee break.

\subsection{Observations}

To reach the company I used the corporate bus, which was also a good place for observation. In the late mornings when I usually travelled the bus was empty, only transporting some young employees, most probably interns or part-time workers. Going home in the afternoon, around 4 and 5 pm, however, the bus was always full and loud with chatting employees. I heard conversations about work, daily issues, concrete tasks and in one case complaints about colleagues, as well as friendly private conversations. I tried to organize the interviews in a way so as to have several in one day. Sometimes I managed and conducted four or five interviews per day, but in most cases at least one interviewee changed the date the previous day, or not rarely at the last minute. Consequently, instead of the five interviews I had planned, I often managed to conduct only one due to the cancellations.

Between interviews I sat in the communal halls or in the cafeteria, where I could prepare for the next interview and observe the environment in the meantime. The infrastructure of the workplace is very pleasant and well-equipped: the building is modern, spacious and light with natural colours and lots of windows. The litter bins are selective, showing commitment towards environmental protection. Colourful and comfortable furniture can be found in the communal areas which serve for relaxing and also for holding less formal meetings. There are no offices, but roomy open units with a small kitchen and several meeting rooms on every floor. The building contains a fitness room for sports, a cafeteria, a canteen and several vending machines. Benches in front 
of the building and a terrace make it possible to take a break outside. Between 9 am and $5 \mathrm{pm}$ there is heavy traffic in the corridors every half an hour when employees run from one meeting to the other with a laptop in their hands. Moreover, there are always some people talking in the cafeteria, making phone calls in the corridor or working in one of the corners of the communal space. According to the interviewees and my own observations, many of the employees leave around $5 \mathrm{pm}$. The cafeteria also closes at that time. Most of the employees are young; the average age may be around 30-35 based on observations. Regarding dress code, most employees wear rather professional and formal outfits, although this may vary according to unit. At one time the company leaders made a speech in the communal area on a mobile, pre-constructed stage and many of the employees spent their time listening to them from the corridors, or from in front of the stage in an enthusiastic atmosphere with frequent applause and cheering. Another time I found a board not far from the entrance advertising stress-reducing massages for employees that day. Although I have no information about how many employees used this service, it can be regarded as a contribution to the work-family balance service by targeting relaxation and stress reduction.

Turning to a description of the interview situations, before the interview I always waited in front of the given meeting room, or in those rare cases when the meeting room was not being used beforehand, I waited inside. Most interviewees arrived on time but short delays also occurred. In five cases I had to call the interviewee after 10 minutes to make sure they had not forgotten our appointment. Delays were crucial because, in general, only 30 minutes was available for the interviews. Due to the busy schedules of managers I could not conduct longer interviews without risking their refusal to contribute, on the one hand, and moreover, all the meetings held at the company (thus the booking times for meeting rooms) are also fixed for 30 minute periods. Because of this I often had to leave the meeting room with the interviewee when the booking period for the meeting room was over, and other employees arrived to use it. These cases were not very inconvenient since we could usually quickly find another room to finish our conversation. Most of the time the 30 minutes allocated for the interview was very tight and I really had to focus the conversation in order to get through all the questions I had planned. In some lucky cases the interviewee did not have another meeting after our interview and could spare some additional minutes to continue our conversation. In this way the longest interview (which was also one of the most interesting conversations I had, conducted with the chief of the Human Resources Department) lasted 47:18 
minutes. There were only very few interviews when the interviewees were not very talkative, therefore we were able go through the interview guide quickly. Consequently, the shortest interview lasted 15:15 minutes. Additionally, one skype interview was held with one of the floor managers who was working abroad on a project. In contrast to the other floor managers who refused to participate in an interview due to the lack of opportunity for a face-to-face interview, this manager accepted to do it online. The technological solution worked well and we were able to conduct the interview without problems.

The meeting room was a private but also natural environment for the interview. After a short introduction I asked the interviewees whether they would had mind if I recorded the conversation using a dictaphone. Only two interviewees - a male and female group manager - refused to allow the sound recording. Some of the other interviewees seemed distrustful at the beginning, but after the first five minutes, after seeing what kinds of questions they could expect, their distancing dissolved. The interview guide did not include sensitive questions. The topic of work-family balance in itself offers the potential to talk about sensitive issues such as family or private problems or critiques of the company, and might raise confidentiality issues in an interview situation, but in my case I left the interviewee to decide how far she or he wanted to go into these issues. I never felt that an interviewee was saying something contrary to his or her opinion, or was manipulating answers. But it is probable that some did not share with me their critical remarks about company support or workload as firmly as others. Where I felt that the interviewee was open to talking more critically, or sharing very personal experiences, I encouraged them, but I avoided pushing these topics. One of the interviewees was a special case in the sense that he had a two-yearold son with a serious central nervous system disorder. After finding this out at the beginning of the interview, I could not continue in my usual role of interviewer, nor with my interview guide, because there were questions which would have been inappropriate in the situation. In this case, I skipped the relevant questions and asked others which focused on his special case. At the beginning I was more embarrassed than the interviewee, but since he was very open to talking and encouraged me to ask questions, we were able to continue with the interview in a very personal atmosphere. As usual in interview situations, some remarkable comments were made after the recorder was switched off. In these cases, notes were made right after I parted from the interviewee. This was how a female interviewee told me about her depression after her 
divorce, and another that she had become pregnant via in vitro fertilization due to her husband's physical condition. This level of honesty had a major influence on me as an interviewer embedded in the research situation.

All in all, the interview situations in general were pleasant, the respondents were friendly and used an informal tone, often joking as well. I did not feel that my gender or my age was significantly influencing the conversations. Of course, this cannot completely be excluded since, as a researcher in the field, I must have affected the observations I was making. But despite the high-level positions of the interviewees they treated me as an equal partner, taking the interview seriously and typically showing interest in the topic. Three male managers started the interview by telling that they had thought a lot about their work-family balance since they had accepted my interview request, and thanks to the research topic they had been more self-reflexive regarding their own behaviour in the past few days. At the end of the interview many respondents asked about the outcome of my research. I promised to send them a summary of the results, which I did at the beginning of September $2015^{15}$.

\subsection{The Company and Work-Family Balance}

Due to reasons of confidentiality and anonymity, the company's general characteristics cannot be described since they could lead to its recognition. It is crucial to state, however, that it is among those companies who find it important to advertise the relevance of corporate social responsibility and work-life balance on their website. Among work-life balance opportunities, they list a broad scale of benefits, corporate culture, part-time and flexible working options and put an emphasis on healthy lifestyles, and promoting sport activities inside and outside the company building. Regarding corporate social responsibility, the employees are the focus on one hand, and the economy and society on the other. Based on the website, the company puts high level of investment into their employees' human capital, health, security and working conditions, as well as promoting their engagement with the company. From the HRrelated conversations I was informed that the Employee Engagement Survey is not the only study the company conducts but there are also surveys about stress and health,

\footnotetext{
${ }^{15}$ Since that a workshop was organized in January 2016 within the company about the topic of more women in the leadership and gender issues in general. I was invited to talk about my research. Unfortunately I could not attend but prepared a power point presentation that I explained via phone to the organizer of the workshop. She was the one holding my presentation and according to her feedback the audience showed great interest in the research findings.
} 
although not annually, as well as surveys about colleague and workplace satisfaction. The importance of diversity and equality of gender, race and age is also stressed. Among the issues of social and economic responsibility the importance of transparency, sustainability, environment protection and charity or social events is highlighted. The company's annual social responsibility reports can be downloaded from the website. Following the findings of Géring's (2014) study, it seems even more important to observe that the company puts significant emphasis on its online representation regarding corporate social responsibility and work-family balance.

The results of the Employee Engagement Survey which were presented by one of the HR assistants also gives useful insight into the company's working environment. The survey was filled out by $98 \%$ of company employees in 2014. The engagement index was $79 \%$ that year, showing high engagement towards the company. The index had several core dimensions, one of them which described the working environment. Responses were very positive in this regard as well: between $80 \%$ and $90 \%$ of respondents strongly agreed or agreed with the statements that the leaders encourage diversity among employees; the working atmosphere promotes trust; and the physical environment is satisfying. Two items did not receive such high ratings however: $63 \%$ of staff members and group managers, $47 \%$ of heads of department, $41 \%$ of directors, and $50 \%$ of $\mathrm{C}$ level managers think that the workload is reasonable. The statement relating to work-life balance produced similar results: $70 \%$ of staff members, $58 \%$ of group managers, $50 \%$ of heads of department, $47 \%$ of directors and $67 \%$ of $\mathrm{C}$ level leaders strongly agree or agree with the statement that they are able to manage their work responsibilities in a way that allows them to maintain a healthy balance between work and home. In both cases it can be seen that the pressure is higher on head of departments and directors, but in general, managers experience a higher level of work-family conflict than staff. The HR assistant also talked about another piece of research which measures whether employees would recommend the company to others. According to the results, $60 \%$ of employees would recommend the company, while only $12 \%$ would not. The other answers were neutral. Those who would not recommend primarily made reference to leadership, to the company guidelines, to the absence of recognition and to the high workload (in descending order). As can be seen, workload was only listed in fourth place and only $32 \%$ specified it as a reason. Only $26 \%$ of the non-recommenders indicated that work-life balance was the reason that they would not recommend the company to others. 


\section{RESEARCH RESULTS}

The summary of the research findings starts with a description of the sample which includes an overview of the distribution of interviews by demographic variables. This is followed by the analytical section, which is divided into three main parts based on the three research questions. All of the three main parts start with a description of manager fathers' answers along the coding categories of content structuring analysis. This material is followed by a summary of female manager responses compared to those of the male sample. The main chapters end with a discussion section which covers the interpretation of research results in the context of the previously mentioned theoretical concepts and empirical findings.

\subsection{Description of Sample}

The sample consists of interviews with 43 men and 15 women. According to their positions, the male group contains 22 group managers, 13 heads of department, 6 directors and $2 \mathrm{C}$ level managers, while the female sample involved 11 group manager and 4 head of department interviews (Figure 2).

\section{Figure 2 Distribution of Interviews by Gender and Managerial Level}

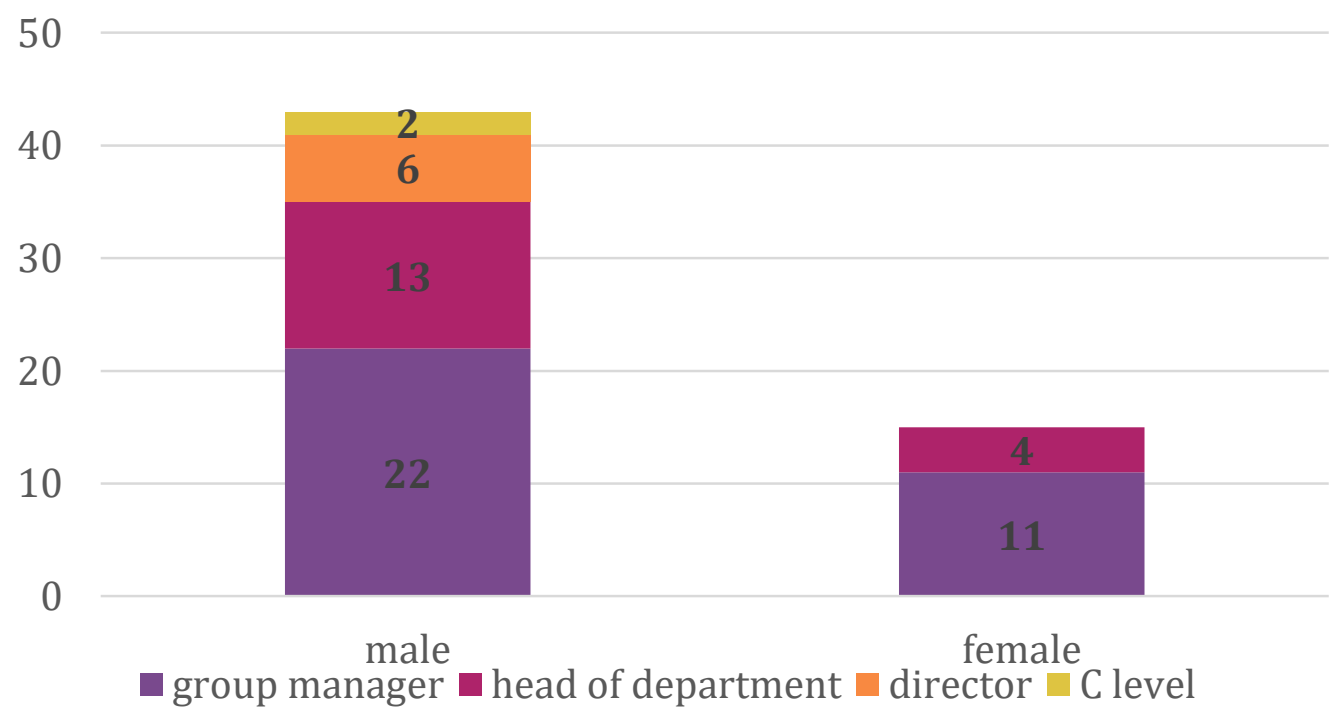

Source: Author's construction

Among the potential interviewees there were also 3 female directors, but two of them were on maternity leave at the time of the research and one of them did not answer my e-mails. Nonetheless, it can be stated that management is rather vertically 
segregated by gender. At the time of the research, there were no female managers in the board, although at the time of writing one of the 7 leaders in the board of management is a woman. Among the male board members is one of the leaders with whom I conducted an interview. The other board members were either non-Hungarians or not fathers, therefore they were excluded from the group of interviewees.

The average age of male interviewees is 39 years, while among women it is 41 years. The youngest man in the sample is 29 years old, while the oldest is 49 . The age range is less for the women: the youngest respondent is 34 years old and the oldest female interviewee in the sample is 46 . The average and modus of number of children is 2 for both men and women. The maximum number of children was 4 , with four male respondents having that many children. Some men in the sample had very young children: a 3-month-old baby was the newest family member. Only 7 men in the sample had only children of school age, while all the other fathers had at least one child of less than 7 year of age in the family. There was only one man with twins. The children of the women were all kindergarten or rather school age, since all the female managers in the sample had already come back from maternity leave. Almost all the respondents are raising their own children; only one father has step daughters from his wife's first marriage. Regarding marital status, almost all the respondents are married. This is especially true of men, as only one of them is divorced and cohabiting with a new partner. Among female managers the picture is somewhat more diverse: 11 out of 15 women are married, while 4 of them are divorced. Among the divorced manager women two have formed new partnerships, while two is raising their children in a single-parent household.

The average number of years employed by the company is 11 . For men the average is 9 years, while female managers in the sample have worked for the company for longer (12 years on average). The managers who have worked for the longest time for the company - one man and one woman - have each been employed there for 20 years, while the newest manager had only been employed for 4 months at the time of the research. In terms of the professional field the respondents are involved in (Figure 3 ), there is great diversity, from finance and marketing to customer service and sales. Interviewees with a background in marketing and information technology are most frequently found in the sample. Although in the marketing unit there are some female managers, information technology is entirely dominated by males. This is not only true 
of the managers who are parents, but the interviews indicated that only men work in the IT unit, besides one female intern.

Figure 3 Distribution of Interviews by Gender and Field of Work

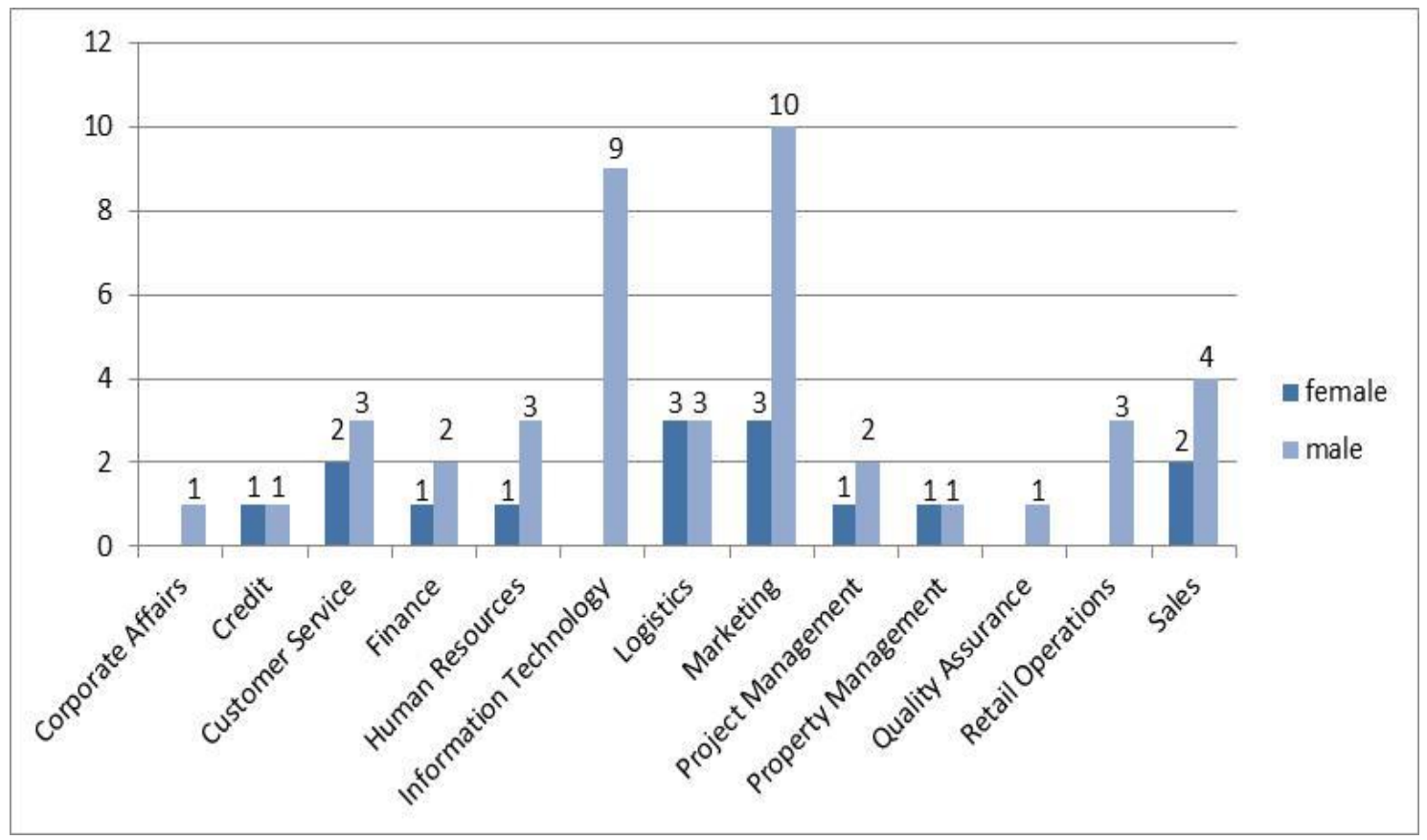

Source: Author's construction

Three male managers in the sample had left the company at one point during their careers but had later returned. One of them explained that he was always looking out for the right position and new, challenging opportunities, not specifically for a particular company. Therefore, when he got a better chance at another company, he moved there, which is the same reason he later returned. As for the other two managers, they had realized that they preferred to work at their original company compared to the other experiences they had had.

Regarding level of education (Figure 4), the sample is unsurprisingly very homogenous. Almost all the respondents have a higher education, college or university degree. The degree topics are primarily related to economics - mostly business and management, and also human resource management or tourism management - but among men there are plenty of technical and IT graduates, as well as those with college degrees as teachers. Most of the women in the sample are economists with a focus on logistics and accountancy, or human resource specialists. Only 4 respondents from the sample (two men and two women) have only a secondary education (high school or technical education). 
Figure 4 Distribution of Interviews by Level of Education and Gender

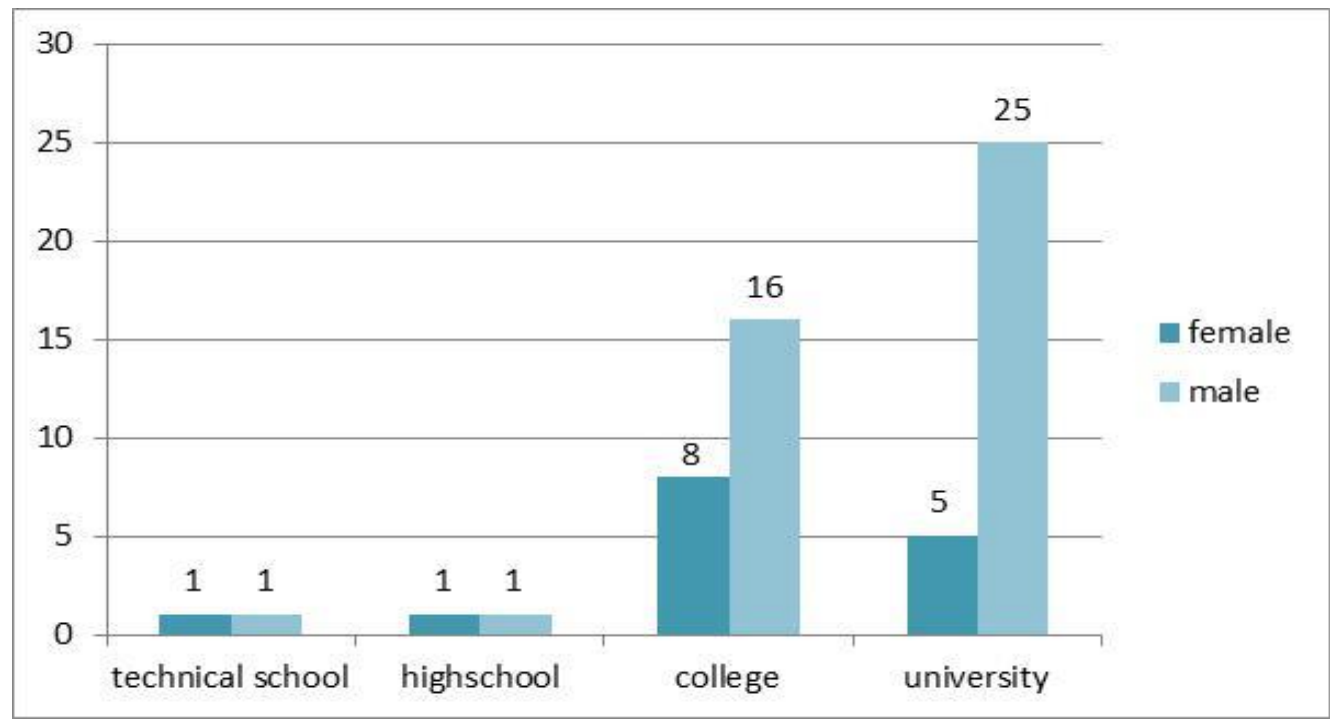

Source: Author's construction

The educational level of the respondents' partners (Figure 5) shows a similar picture: most of the interviewees' partners have a college or university degree. The field of the degree, however, is more diverse than in the case of the interviewees. Many of the wives of the male interviewees have gained certification in economics, just like their husbands, in the field of marketing, finance, human resources or accountancy. But there are also graduates in the liberal arts, as well as a dentist and a dancer.

Figure 5 Distribution of Interviews by Partners' Level of Education and Respondents' Gender

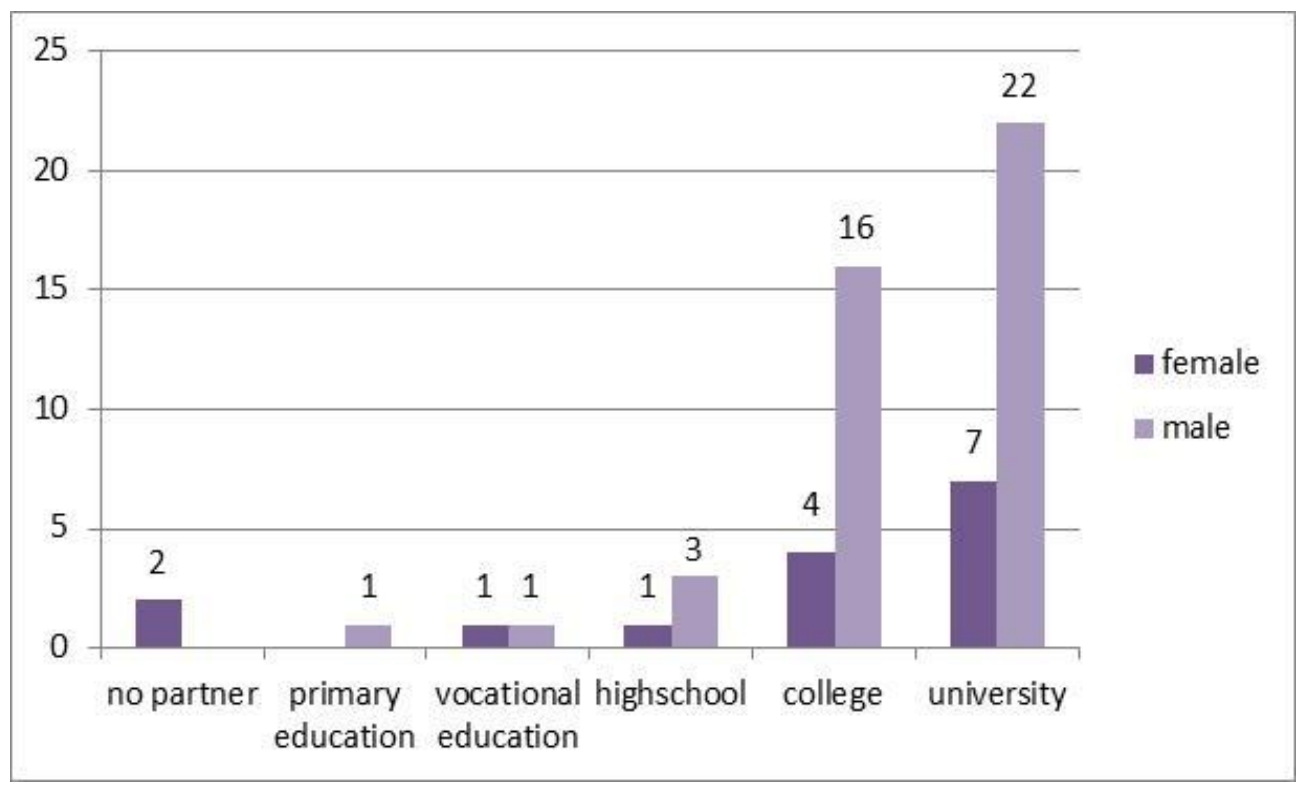

Source: Author's construction 
In the case of female managers, partners or husbands often have technical or engineering-related certification. Only 5 managers from the sample have a partner with only a secondary education, while one manager's wife has only primary education.

Regarding the nature of the partners' occupations (Figure 6), there is wide diversity by gender: one-third of the male managers' wives were on maternity leave at the time of the research. No men from the sample have taken any parental leave beyond the 5-day paternity leave. Another third of male interviewees are part of dual-earner couples and their wives are also working full time, while 6 of the male interviewees' wives have a part-time job, resulting in one-and-a-half earner couples. There are 6 male managers whose wives are self-employed (including a manicurist, a tattoo artist and a dance teacher, for example). As for the female interviewees, all of them with a partner are part of dual-earner couples who have a husband or partner occupying a full time position, or in one case working on a self-employed basis. The partners of 4 male managers and 2 female managers are also working at the company under investigation.

\section{Figure 6 Distribution of Interviews by Partners' Type of Occupation and Respondents' Gender}

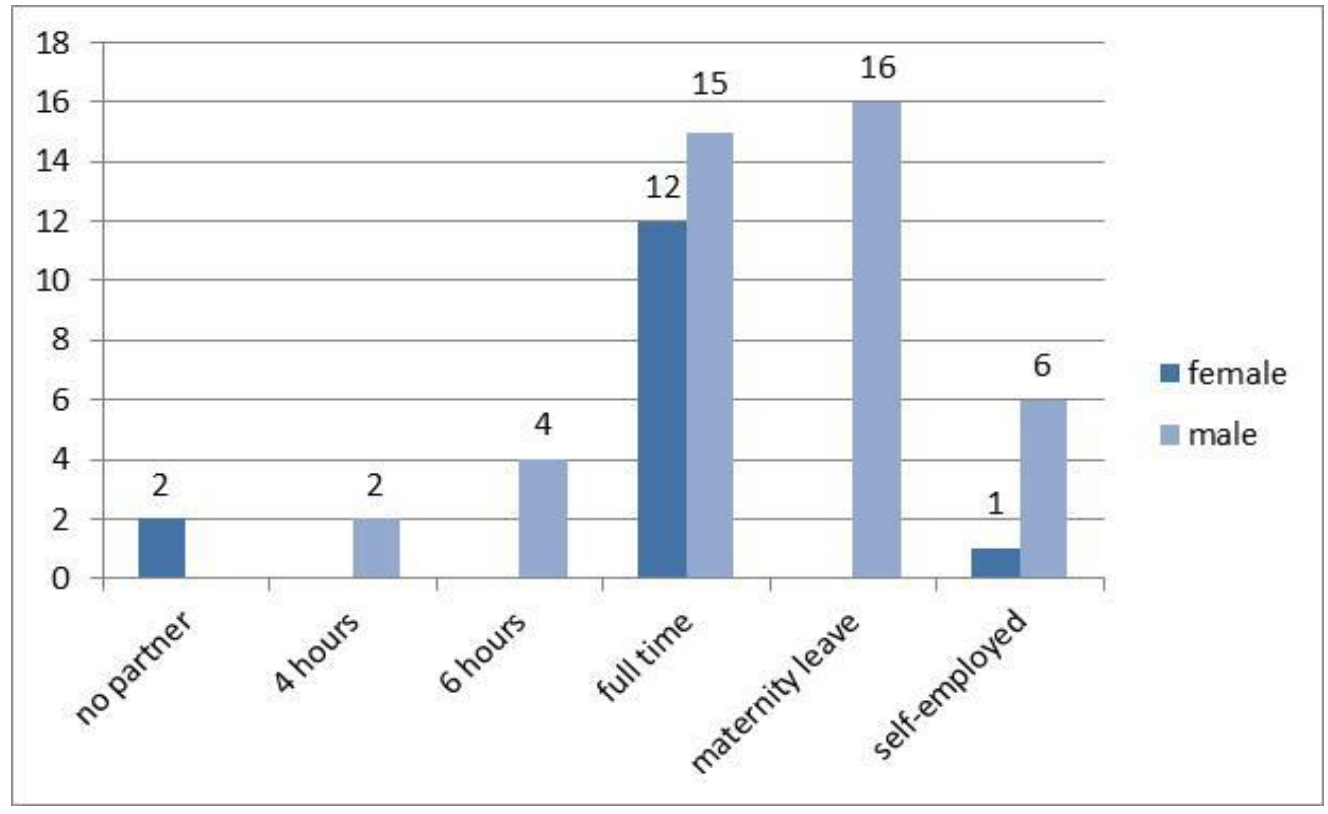

Source: Author's construction

\subsection{First Research Question: Work-Family Conflict}

This chapter, which is connected to the first research question, describes how managers in the sample perceive their work-family balance and what problems they have to face. The structure of this section is the following: it starts with a description of how manager fathers evaluate the current state of their work-life or work-family balance 
and what they understand by these terms. In the case that they experience conflicting demands between their work and family life, what are the sources of these problematic situations, and what consequences do manager fathers experience? This chapter includes a description of the adaptive strategies manager fathers use to harmonize their work and family responsibilities. This is followed by a summary of the female sample responses and a discussion related to the first research question.

\subsubsection{Evaluation and Meaning of Work-Family Balance}

I started the interviews by asking the respondents how satisfied they are with their recent work-family balance. Regarding how manager fathers from the sample evaluate the balance between their work and family life, we can see a mixed picture. Three distinct groups of respondents emerged: manager fathers who are relatively satisfied with their work-family balance; those who are critical about the current state of their work-family balance; and those who have both positive and negative feelings.

Around one third of all male interviewees (including all levels of management) are rather satisfied with their current work-family balance. Some mention that a perfect balance does not exist, and sometimes they face difficulties of course, but on the whole they are satisfied with the balance in their life. A few respondents emphasized the company's role in their work-family balance at this point in the interview, stating that the firm offers every tool - in the first place, flexible working options - to support the balanced living of its employees. Other interviewees compared their recent situations with their previous working experience, concluding that their work-family balance had significantly improved by changing workplace and choosing this company. Many manager men chose to highlight their own responsibility for achieving work-family balance through pointing to the need to be conscious and pay attention. According to these respondents, balance has to be a personal decision and one has to invest much effort in accomplishing and maintaining it.

Another third of male respondents were critical of their work-family balance and referred to lasting problems. In every case, the source of the problem was connected to work. These respondents feel overloaded and unable to finish their work within 8 hours; a situation which results in them doing constant overtime and regularly working long hours, even in the evenings or weekends. They find that resources and duties are not proportional, so there is a huge pressure and workload on most employees. The problems in some cases derive from concrete situations, such as an employee becoming 
responsible for many new clients, or being promoted to the position of director while still occupying the former position until a new employee is found. For a few interviewees it was not the amount of work that was the problem but the distance between the workplace and their homes. These individuals have to spend a daily minimum of 2 hours commuting.

Next to rather positive or rather negative evaluations of work-family balance, the last third of manager fathers reported to having mixed feelings. They highlight the cyclical character of the company's functioning, having busier periods with high workload and overtime, but also average amounts of work during peaceful periods. They consider this a natural characteristic of the market. This does not mean that the tough periods are easy to endure, but they can at least see the end of these phases, knowing that they are only temporary states. Because of this situation, some interviewee added that they might have answered completely differently if they had been asked one month or even two weeks earlier. Four manager fathers reported to seeing improvements in their work-family balance rather than fluctuations. In every case, the improvement was achieved by personal changes in attitude like becoming more conscious about prioritization and planning, or in one example by the concrete act of moving closer to the workplace to save on commuting time.

Respondents' answers about how they evaluate their work-family balance gains real meaning in the context of what they actually think the term work-family balance means. People can interpret this word in many ways, as it transpires from the interviews. Several potential categorization schemes could be used to classify these answers based on the elements of time, level of stress, availability, work domain, home domain, private life, etc. In this case work-family balance definitions were classified as the following: approaches from the perspective of the family; approaches from the perspective of work; balance between life domains; lack of conflict; and flexibility. Although later during the interview I focused my questions rather on work and family life, as the research indicates, I used the term 'work-life balance' in the interviews instead of 'work-family balance' in order to avoid narrowing the topic and losing useful information. In comparison, almost all the interviewees' responses about the meaning of balance in their life concerned the domains of work and family.

One quarter of the manager fathers approached the definition of work-life balance from the side of the family. According to this approach, work and family are in balance when the managers have enough time and enough quality time with their family and a 
feeling of being available for them without constantly thinking or worrying about work. Thus this approach can be understood primarily from the perspective of time and devotion: "If I asked my family whether I spend enough (in terms of the amount and quality of) time with them, they would say "yes, this is acceptable" (...) The amount is that it would be good to arrive home around 6 every day and spend time with my children and wife before going to bed. And to be together on the weekends. And in terms of quality, that one is not exhausted at those times, but still fit." (chief, man, 43) The term 'quality time' that was often used was explained as complete devotion and attention to the family during the short time spent together. Besides describing the need for more quality time in general, some interviewees formulated more concrete desires about being present in their children's lives. This related to attending important events such as school programs, competitions, sports-related training, or simply being able to pick up children after school. As mentioned before, only a few respondents talked about hobbies and private time as components of their work-life balance. The reason is that devoting satisfying time, energy and attention to their work and family life is so challenging in itself that there is hardly any room left for themselves and their hobbies. Therefore many interviewees consider hobbies options which are simply 'nice to have'. Consequently, they did not mention them in their understanding of work-life balance, but they turned up later during the interview when I directly asked them about sacrifices they made and what was lacking in their lives.

Another big group of respondents, a quarter of manager fathers, defined work-life balance very similarly, although approaching the term from the perspective of work. Their view about work-life balance implicitly includes the desire to have more private life or to spend more time with their family, although they emphasize the characteristics of work. For most of them, balance means finishing their jobs within normal working hours without overtime, bringing work home or worrying about unfinished duties.

Less than quarter of manager men defined work-life balance in the classical way, by imagining a weighing-scale with two equal weights. According to these interviewees, they feel balanced when work and family relate to each other in a proportional way, meaning that they can perform and maintain both domains equally well. Thus, this approach to work-life balance includes time that is devoted to specific activities "How many hours I spend with work and family. If the time spent with work is significantly more than 8 hours, it's not good" (group manager, man, 36), presence and concentration "If I have to put it into one sentence, to me balance means that I am 
where I have to be. In mind, in everything." (director, man, 40), as well as performance. It usually also involves the separation of life spheres by paying undivided attention to the domain they are physically and psychologically present in at the given moment: “Well, listen, when I'm working I'm concentrating on my work, when I'm at home, I relax completely and I care only for the children and family." (head of department, man, 40)

For some interviewees, the work-life balance is equal to flexibility. In contrast with the previous definition (in which work and family were seen as separated domains), those who see balance as flexibility consider the domains to be permeable. They do not think that equal proportions of work and family life are necessary, or that too much work ruins the balance. They rather emphasize a general feeling or state of satisfaction that they are in control of their life and can accomplish every task they want to. This does not depend on the number of hours spent on work or with family, or even physical presence, but on performance. They consider flexibility to represent the freedom which allows them to perform even without being present, or to adapt to emerging situations, as well as being able to prioritize: "I'm very lucky from this point of view, because I work flexible hours, which means that I have to perform on average and I have the option to have half a day off when I need. Thus I form a balance in a way that the kids do not lack anything." (group manager, man, 40). A few male respondents described work-life balance as a kind of positive spillover. According to this view, work is not seen as 'rival' to family life, but part of one's identity. Therefore dealing with work, even if it takes extra time from family, can result in satisfaction and a good mood which spills over to the home domain as well contributing to a general well-being: "The work, the projects, the tasks we are doing here are at least as important parts of our lives as family. Therefore if we can enhance this it will positively contribute to my life, then it is a balanced thing." (group manager, man, 39)

Finally, a few interviewees defined work-life balance as a lack of conflict, when balance means an absence of a sense of guilt or stress. Two interviewees approached the definition from both directions; namely, that work does not continue at the expense of family life and family does not continue at the expense of work. They also add that the problem is not when imbalance between work and family happens occasionally, but when it becomes a constant state. The other respondents find the direction of work-tofamily more problematic and define balance as a lack of guilt towards the family: "For me, balance is when my family is not damaged because I work too much. This partly 
explains why my wife works 6 hours. We agreed that someone has to care for the child. It's impossible that the children are raised by grandparents or a babysitter because this is not a healthy thing. " (head of department, man, 33)

The issue of the direction of conflict between work and family emerged in two ways: on the one hand, interviewees were asked about the direction of conflict (whether work intervenes with family life, or family life interferes with work, or perhaps both phenomena appears simultaneously). The other component of direction relates to the use of information technology (whether technology helps to arrange private matters at work or enables work to take place outside the office). From the perspective of conflicts, all the respondents mentioned that work influences their family life more than the reverse. This does not necessarily generate conflict in every situation, but thinking of work while at home is a frequent phenomenon in most households. In contrast, most of the manager fathers can easily leave their private lives outside their workplaces. This can be explained by the fact that the pace of work is so rushed that employees do not usually have time to concentrate on other things than work. Another factor which makes private life more separable from work is that most of the male leaders have order in their family lives thanks to partners or other family members taking care of domestic duties. In the case of important or emergency situations, however, family takes precedence, especially when a child is sick or a family member is in trouble: "To be honest, I don't have the capacity and time for these everyday things of life at home, like whether I have read the gas meter or what I did with the last invoice, or whether I really did turn off the iron, because in the given life situation they enjoy far less priority than anything that happens here. But not a long time ago my wife's grandmother was very old and ill and, no surprise, she died. And for that day this momentarily overrode everything happening here in mind and time a bit." (group manager, man, 36). Only very few male interviewees talked about mutual interference between work and family life. They see their life in a holistic way without making a clear distinction between themselves as employees and their private selves "I am a private individual and a professional employee at the same time who sells his knowledge for a salary. And this is a good deal. I'm here because I find it a healthy exchange. But I'm a private person at the same time and I can't switch it off. It's completely normal. They don't interfere, they merge." (director, man, 40), while two managers emphasized the mutual conflicting impacts of work and family life: "Yes, I'm the type of guy who doesn't separate these things. If there are private problems, not necessarily relational private problems, it could be let's 
say, buying a flat, or financial problems, or anything, then of course they have an effect" (group manager, man, 38).

When we take into consideration the other dimension which was mentioned, namely the direction of technology usage between work and family, we can see a similar pattern. Using a phone or a laptop for work purposes at home seems to happen more frequently than using the devices for private matters during working hours. Compared however to conflict, information technology usage is more mutual between work and family, even if the family-to-work direction happens less frequently and is rather used for administrative everyday issues like paying bills online or making administrative calls. In order to arrange private matters like seeing a mechanic, arranging a meter reading or having the chimney swept employees have to take advantage of a home office or flexible working hours. More private issues were also mentioned, such as ordering Christmas gifts or checking a website selling prams that a wife sent a link to, but these occurred more rarely than everyday administrative responsibilities. Very few male interviewees have an implicit agreement with their wives that they can be called during working hours only in emergency situations, otherwise they do not like to be bothered while working.

\subsubsection{Sources of Work-Family Conflict}

After briefly describing what the manager men understand under the term workfamily or work-life balance, and detailing how satisfied they are with the current state of balance in their life, let us move now to the next step of investigating what the factors are which cause problems in the lives of managers. As could be seen earlier, around one third of all manager fathers consider their work-life balance to be satisfying - those who rarely mentioned conflict situations or only those connected to time pressures - while two thirds of the respondents faced certain problems either temporarily or in the long run. Although the core of this research is work-family conflict, I tried to avoid forcing the issue of conflict on respondents during their questioning. This is also the reason that I did not start the interview immediately by examining the conflict dimension but tried to bring it out by asking generally about employees' work-life balance satisfaction. Those respondents who experience or have experienced difficulties in the past mentioned this straightaway at the beginning of the interviews, therefore it was easy to continue along this topic. The conflict sources are categorized along the three types of 
Greenhaus and Beutell (1985); namely, time, behaviour and strain, and are now described in detail.

The most frequent type of conflict was time-based conflict, and, as was explained in the previous chapter, work-to-family direction. This means that in most of the cases when respondents found their work-family balance to be problematic, the conflicts that emerged from work prevented them from spending as much time with their family as they wanted. As mentioned in the conflict definition of Greenhaus and Beutell (1985), time squeeze not only relates to physical absence from one life domain, but also a lack of psychological and mental involvement in the current life sphere. These two components of time-based conflict both appeared in the interviews. On the one hand, about two thirds of manager men complained about their actual workloads, including tight deadlines, overtime, working during the evenings or even on weekends and holidays. Many of the interviewees emphasized the cyclical character of their workloads. Before Christmas in November and December, every employee knows that high-paced work can be expected. Some interviewee consider this to be characteristic of the private sector and can deal with it. Still, more than half of the manager men find their workloads during these periods of rushing to be extreme. During these times, the average number of hours worked per day is between 10 and 12, including weekend work as well. It seems, however, that the interviewees can better handle these situations when they are warned in advance and are prepared to work longer hours, compared to unexpected situations. Those men who considered their work-life balance negatively however complained about the constantly high workload not only during these intense cyclical periods. In some cases, this may be explained by the type of department that the employee works at, such as property management, where constant availability is expected. The general problem however is a lack of human resources. Some directors formulate it clearly that the secret of the company's exceptional performance lies in the fact that employees are required to undertake more work than would normally be expected of them: "I have bad news: whatever we [as the company] are saying here about how much the company does for it, it's a strong expression, but in fact we are exploiting people. Strongly. (...) And all the things [organizational work-life balance arrangements] we try to do stay rather on the surface." (director, man, 42). There are differences in perceptions about the level of management. A few group managers were complaining about the double squeeze of requirements they experience from below and from above - their superiors. Some other group managers however had the perception 
that the workload increases as one goes up the hierarchy, and they would not want to shoulder the burden that their bosses do. The directors and top leaders do not feel that they have actually more work or tasks than their subordinates; however, they perceive higher levels of stress due to the higher level of responsibility.

Besides the lack of human resources, the significant workload can be also explained by a meeting culture. Since this meeting culture is a basis of criticism within the company, it is described in detail later. What is important to know now is that due to constant meetings during the day, managers do not have enough time to catch up with their operative and administrative duties. Since they cannot accomplish these within working hours, they have to physically take work home and complete it in the evenings or early in the mornings before coming to the workplace. This leads to the other component of time-based conflict; namely, when someone not only brings work home physically, but also in his or her mind, preventing the individual from relaxing or paying attention to the family. When these managers open up their laptops at home and continue working it is obvious that they have remained in their working domains, even if they are physically already at home. This prevents them from paying attention to their partners or children, enjoying the time they spend at home. Some men do not find this necessarily problematic because they see it as part of modern life and working culture. They are aware that in the service sector one cannot drop work and immediately exclude it from their minds. A few interviewees used examples to illustrate their argument by comparing their work to a factory job where one can forget about work after leaving the assembly line. They consider, however, their own white collar service sector work as something which can never be finished, but only interrupted: "You can't exclude this. Already in the morning, sitting in the car, I'm thinking about having two meetings and I'm already preparing in my head." (head of department, man, 40). Most of the manager men in the sample, however, consider it bothersome if work affects their social relationships: "You can't switch off. It's not that kind of job where you leave at 5 pm or 7 pm or whatever and after stepping out the gate you have already forgotten any kind of work-related thoughts. I think it's much more harmful that I can't switch off and I can't concentrate on my family even when I'm with them." (head of department, man, 39)

A third source of time-based conflict also emerged from the interviews which is not connected to workload or to work occupying one's thoughts. This is the distance between the home and the workplace. Currently, only some men are concerned with this or had similar problems before. For those who live far from the company, the daily 
commuting time varies from 2 to 4 hours. Therefore even in those cases when they can finish work on time they have the perception of working long hours since they arrive home late and have less time with their family. Moreover, further logistical problems arise since it is impossible for these interviewees to take their children to school in the morning or to pick them up at the end of the day, and even offices are closed when they need to arrange administrative issues. Two of the affected interviewees tried to find the positive side of this situation and use the long trip to arrange phone calls, or to think over the day. The others, however, really found it problematic. They see it as a stalemate since they do not have better working opportunities in their home towns, but they do not intend to move closer to the company since they are emotionally attached to their homes or have relatives living nearby. One of the male group managers solved the problem by moving closer to the company; consequently, he no longer faces this pressure, while a floor manager (an expatriate formerly spending only weekends at home) decided to move abroad with his family for the length of the project.

The second conflict type in the typology, behaviour-based conflict, occurred least frequently and its consequences did not seem as significant or were at least less visible than those of time-based and strain-based conflict. Based on the answers, two forms of behaviour-based conflict can be distinguished: the first one emerges from the pressure of simultaneous expectations of the work and family domains, while the other is derived from the difficulty switching between certain roles. According to contrasting expectations, fathers can face the double pressure of being a 'super dad', as one of the directors put it, and a successful manager at the same time. A quarter of male managers experienced internal conflicts with meeting the requirements of fulfilling a traditional breadwinner role and a more emotional, caring father and partner role. In some cases exhaustion makes them unable to perform their roles as fathers or husbands, since after a tough day at work they do not have the energy to play with a child or to listen to their wife. One of the directors had conflicts with his wife because after his exhausting work he did not appreciate the housework and care tasks his wife accomplished at home. Other fathers complained about their lack of capacity and time for performing in both life domains. One of the heads of department perceived external pressure from his wife which he experienced as a paradox between the traditional breadwinner and the active father role: "It's a strange paradox, many of my male companions face it, that we should "earn much more but be home a lot!". Well, it's not possible. (...) I feel very schizophrenic about this. The financial side dominates, the provision of financial 
security, but I think the role model role is even more important. (...) But the whole story does not work if I can't provide the financial security besides this. And it's difficult, because for financial security you need intense work, which takes time." (head of department, man, 35) One of the group managers and a director added that they would find it useful to receive the help or guidance in fatherhood that they got during their work. Other fathers also mentioned insecurity or puzzlement about child-rearing and their own performance as fathers. A director identified the difficulty around the birth of the first child by highlighting the changed status within the family fathers have to adapt to: "Fathers are in a special situation, like mothers are, only in different way. They actually become a service crew in the first three months, losing their previously stronger position. It is very difficult to come to grips emotionally with this, and there is not much help with it." (director, man, 40)

The other form of behaviour-based conflict emerged in relation to the difficulty of switching between roles. This was not a core problem; it concerned only some manager fathers and appeared only occasionally. It can happen that these men remain in their manager roles and use a commanding style of communication not with children but with wives: "The leader role is a leader role. And yes, my wife used to tell me that she was not my subordinate because I tend to communicate that way. Of course we have some tension about this but nothing serious." (group manager, man, 33). One of the male group managers has a wife who is also occupying a leader position. According to him, they had to learn to communicate with each other since both of them are problemsolving driven individuals due to their management positions. Sometimes, however, they do not tell their problems to each other to receive advice and a solution, but only to be listened to. They both had to learn when their partner needs only to be listened to and when she or he needs advice.

The third type of conflict is strain-based conflict, which also emerged in the interviews. Around half of the interviewees mentioned that strain and stress appeared at certain periods of their lives, for some only occasionally, while for others it represented a serious problem. Strain-based conflict is similar to psychologically time-based conflict in certain aspects since these are both connected to a mental state. This fact was noticeable from the interviewees since these types were often merged together by respondents. This is not a problem from an analytical perspective, since as Sebők (2015) explained, work-family conflict categories cannot be considered ideal types. The interviewees talked about different kinds of stress in their lives. On the one hand, a 
general feeling of tension and agitation could be distinguished which lacked direction and was derived from the double squeeze of family and work and a lack of opportunities for relaxation. This is somewhat similar to behaviour-based conflict, since the individuals feel that they lose themselves in trying to meet all the work demands and expectations of family members. Having a lack of time for one's own hobbies and relaxation was a general problem for almost every interviewee, but three managers articulated very clearly how it increases their stress level, causing constant tension and endangering their health: "Everyone needs special stress management. I'm bad at it. I can't switch off even on holidays. My wife often tells me that it's totally useless to go with me on holidays, because I'm not present with my family mentally, only in body. (...) I have to form a life strategy regarding this if I don't want to be killed by stress at 50." (director, man, 42)

Besides this general feeling of stress, work-to-family direction (namely job stress) was observed. In some cases interviewees found it normal that they felt more stressed or agitated when deadlines were tight or more projects were on the line simultaneously. When such stress is temporary or does not influence significantly their mood or relationships with their family members then it is not considered a serious problem. Job stress can also derive from one's own perfectionism. Many manager men complained that they were increasing feelings of stress themselves by trying to deliver every task perfectly which is often impossible in the rush of simultaneous projects and deadlines. One of the top leaders explains this tension by pointing to the abovementioned lack of human resources which puts extra pressure on every employee. As mentioned above, there seems to be a difference between levels of management regarding the risk of stress. Higher-level managers face greater responsibilities because they make decisions that influence groups and divisions. This makes them more vulnerable to psychological pressure: "I don't think that a director or cxo has to tolerate a bigger workload during working hours or in kilojoules, but the workload is different, it's rather stress. (...) The emotional load is definitely higher, there is no question, since we have to take decisions about others' fates, not only ours." (director, man, 40) Job stress seems to represent a real problem in these cases when it manifests in projection. According to some manager fathers, they project their tension either onto their partners or children by shouting, using a strict tone or being impatient. In the mentioned examples the managers realized immediately what they had done and had a sense of guilt about it. The partners of the respondents usually indicate their 
dissatisfaction in such situations but the children are victims of the strain, and do not usually understand their fathers' behaviour. Therefore the interviewees feel even more remorse when they project their negative feelings onto their children. One of the directors emphasized the importance of proper communication in these situations. He stated that he believes that if one can explain their feelings and worries to a partner or even to a child, it can take the edge off tense situations.

\subsubsection{Consequences of Work-Family Conflict}

The previous sub-chapter described the antecedents of work-family conflict in manager fathers' lives. These existing conflicts (regardless of whether they are time-, strain- or behaviour-based work-family conflicts) have different consequences on the individual's general mood, the atmosphere at home and relations with relatives. Based on the answers, the most frequent consequences of the attempt to harmonize work and family life are manifested in a sense of guilt, exhaustion, abnegation, problems with children and arguments with partners.

As already covered, a sense of guilt is a common reaction to having a lack of time and energy for the family, or to projecting one's own stress onto family members. The most serious feeling seems to be remorse towards the children. Almost every respondent could relate an experience about arriving home too late, missing a significant event in the child's life, or missing being able to play with them due to work. "Of course, when my daughter says in the morning "dad, let's cycle in the afternoon!" I can't promise to do it. Or when I'm working from home, and they see me and expect me to take care of them, but I have to say no, because I have to work even if I'm not in the office because I have to finish my tasks. Then yes, I feel guilty." (group manager, man, 36) Some do not address this as sense of guilt but rather as 'bad feeling' and do not consider it to be a permanent state of affairs. Others try to compensate for these situations by spending as much time as possible with children on weekends, or making their time together 'quality time'.

Fewer men mentioned that sometimes they felt guilty about their action towards their partners. Usually this derived from an attempt to focus on the child as much as possible, resulting in neglect of the marital relationship. As one of the group managers put it, when the child is small the father role becomes over-prioritized, and the husband role under-prioritized. Only one male group manager talked about having feelings of remorse towards his own parents. He was worried about his parents growing old and 
getting sick more often while he is unable to visit and help them as frequently as he feels he should.

Besides having a sense of guilt towards the family, less than one quarter of the respondents spoke about remorse towards their work. Some referred to it as a past phenomenon since they had learnt how to get over this feeling, while for others it is still an everyday source of tension. This is connected to perfectionism and the respondents explained it by pointing to their own attitudes of always trying to do their best. Since workloads are high and multiple projects run simultaneously, managers often feel that they could have done a better job if the deadlines had not been so tight. The majority of the sample has experienced this feeling; they accepted however that this was characteristic of their sector and had learnt to limit their unachievable expectations towards themselves. In a very few cases, rather at the higher level management, a sense of guilt towards work appeared in connection with a higher level of responsibility. These managers feel responsible not only for their jobs but for their colleagues and the teams they are leading. If they fail with a decision or do not do their best, it affects the performance of many other employees.

Another consequence of work-family conflict is the exhaustion that was explicitly mentioned by a quarter of respondents. This appeared in the interviews in two forms: on the one hand it is connected to having children. In the first period after childbirth parents cannot sleep through the night undisturbed, which naturally results in them being tired during the day. The other form of exhaustion is also related to the family but in the way that fathers often sacrifice their own time for sleeping or relaxing in order to spend more time with their families after work.

Besides sacrificing their own sleeping time for the sake of a better work-family balance, most managers give up other things too such as hobbies, friends and private time. According to the respondents, they place themselves last on the list of priorities so as to be able to meet the expectations of family and workplace first. The majority of managerial men emphasize that this is not thanks to work, but rather to the change in the status of the family after having a baby. Being a father means making a lot of compromises, a fact which is regarded as normal by most of the respondents. As one group manager reported, one has to accept they must give up certain things to keep a balance, and one cannot expect to continue their old life. While most of the interviewees had a normal social life before their first child was born, they had to change their habits after they became fathers. Organizing meetings with friends became a challenge. 
Instead of going out alone with friends, the interviewees now preferred to spend time with friends who also had a family so they could organize free time activities together which would be enjoyable for both the parents and the children. This is not only true of broader social life but also of relationships with the partner. Romantic dinners and going to the cinema and theatre are taken over by family activities such as hiking and cycling. The interviewees stated that they missed being together with their partners alone, especially during the first years when their child were young and needed constant attention. Later, when they could arrange for babysitting, these free-time activities could be brought back, but without any spontaneity since every step had to be planned in advance.

The other part of life that changes after one becomes a parent is engagement with hobbies, and primarily sport. Many managers regularly did some kind of sport before they had a child, but after could no longer find time for this, or much more rarely than before. Even those respondents who are satisfied with their work-life balance witnessed changes in the frequency of sport in their lives. Some others missed being able to relax and have some private time when they could read a book or just be alone in silence and think. The scale of giving up things after becoming a parent varies, however. A few interviewees reported to feeling a huge lack of time and complained that they had no time for themselves, no hobbies and their whole life revolved around a work-family tread-wheel. The majority, however, thought that it was true that they had had to change their routines by giving up or reducing the frequency of some of their former activities, but they saw this as the normal consequence of having a baby. They could adapt to the new situation by creating new habits, rescheduling or planning more. They also add that one does not need to give up activities forever; this time can be a temporary state, which is part of the balancing act. "If you really want, you can solve it. Before I had a kid I was doing workouts 2 or 3 times per week, I stopped after work to have a swim and loosen my muscles, then I went home. The kid arrived, so my evening is always spent with her now, because this block is mine, this is father time. But no one said I couldn't start my day earlier than I had used to." (group manager, man, 29) A very few respondents even stated that they believed that one does not have to give up old preferences alongside having children. For example, some fathers involve their children into their hobbies when the children are bigger. For instance, they take their children swimming, cycling together or even to rock band rehearsals. 
Another consequence of balancing work and family life can appear in conflict with the partner. According to the male interviewees, their wives are very supportive towards their careers. There may be dissatisfaction when the managers arrive home late from work, or they project their stress onto their partners, but usually the women in their lives accept or at least tolerate the inconveniences that accompany their husband's work. There are, however, cases when the respondents' wives really give voice to their dissatisfaction; these happen usually when managers often have to travel abroad, have to work during weekends and vacations, or cannot make it to be with a child during some of their activities. According to some manager fathers, conflicts are more frequent when the wife stays home on maternity leave. As they explain, the sense of time of those who stay at home is completely different from that of those who are working. Their wives often call them with issues which they do not find important at all, thereby bothering them at work. These wives also expect them to appreciate the housework and care duties they are responsible for, but a few men have trouble seeing this work as equal to the jobs they do. It is important to add that this phenomenon was mentioned only by few male managers. Conflict is also caused when these men do not always have the energy to share in the housework and child-care, although their wives would need some time to relax after being home the whole day only with the child. In three cases the interviewees also reported that they wanted to help their wives by hiring a cleaner, but the wife refused this. It is important to see that these men, and also some other interviewees who already employ a cleaner, consider these paid services to be a help for their wives, not for themselves or for the whole family. "I would be very comforted if part of the housework was done using such help [of a cleaner], and we could do something more meaningful instead. This results in conflict because I back out of housework." (group manager, man, 39)

Finally, a last consequence which was mentioned in the interviews was connected to conflict related to children. Young children present completely different challenges to parents than school-age children. Men who have children around the age of 10 often complained about the period of early adolescence. The main problem is that they do not have the time or the option of knowing everything about their children's lives and are afraid of the things they cannot control through their parenting. A few fathers complained about the bad influence and morals of classmates, fearing that their children would prefer to follow them than the guidance of parents. Similarly, respondents are afraid of the bad influence of the media, cartoons and computer games: 
"There are conflicts and fights because they [the children] are really taking to the telly, to electronic gadgets, to computers, and we have conflicts about this. For example, I switched off the electricity on Saturday so they couldn't have access to it, and I sent them outside to play in the garden. There was some sulking but they played the whole afternoon outside in the fresh air and had fun." (group manager, man, 43) There is a fear as well that they will miss some parts of their children's lives because they are not present, or the children will not need them to be there later. The classic example is when the child does not tell the parent what happened in school. "So when I get to know from the mother of my smaller daughter's classmate that my elder daughter is going to a poetry contest in two weeks' time (which I never heard about), then I feel a bit stupid." (group manager, man, 42) Those few fathers who mentioned this problem seem to be trying to at least catch up on the weekends because they do not want to make their child feel neglected due to their responsibilities at work.

\subsubsection{Strategies for Balancing Work and Family}

The interviewed managers have different approaches to balancing their work and family lives. In attempting to categorize these strategies, one very visible distinction emerges: on the one hand there are those managers who enjoy the flexibility of the borders between work and family and use this permeability to achieve a satisfying balance. On the other hand, some prefer to separate the life domains. This observation is completely in accordance with the two main boundary management styles: integration and separation. These two groups of people, however, are not so easily distinguishable since the same cannot be said when we compare the flexibility of roles with the flexibility of time and tools. Consequently, a third group of employees can be included who use a blend of integration and separation approaches, just as described under the category of 'altering' in Kossek and Lautsch's (2012) typology. In addition, there are two other individual strategies regardless of boundary management styles: one can be called conscious planning or prioritization; the other, social support. Naturally, more strategies might be used at the same time, since these are not mutually exclusive categories.

More than third of manager fathers strongly prefer a strategy of integration. Their basic argument is that in the 21th century and the application of developed technology, work and home are not strictly divided since we are no longer bound to physical places, everything is available via internet and work can be done everywhere. 
Some managers further explain this using the holistic view that an individual does not have to be categorised as employee, parent, partner or friend, but engages in all these roles in a single personality. High engagement with work also characterizes these kinds of respondents; thus, for instance, checking e-mails is not considered to be work but a kind of a hobby or interest. Therefore they do not see the need to construct borders between work and home. Besides this more abstract reasoning about wholeness and identity, most of the flexibility interviewees referred to related to everyday, practical advantages. By flexibility they mean flexible working hours, home offices and technological devices which allow them to connect to the company system from a distance. These opportunities which are offered by the company are considered tools of freedom and autonomy. Managers can arrive late to work if they need to take their children to school, or can leave earlier to pick them up. A home office can be used if a child is sick, or the gas meter-reader comes. These managers are grateful for these options since they have the autonomy to reschedule their day freely, without asking for permission. They highlight the informal ways of using these opportunities without being called to account for their absence. "For me, flexibility is opportunity. For others, it is responsibility, but for me opportunity. I would encounter many problems if there was no flexibility, if I couldn't work from home, if I couldn't reach my e-mails via phone. Let's say I had a little cold, I was feeling a bit sick or coughing, I wouldn't go to work. Then I should go to see a doctor, take sick leave, officially I couldn't check any e-mails, I couldn't handle my tasks and decisions would not be taken." (group manager, man, 29) What makes this possible, according to respondents, is a focus not on hours worked, but results. As these interviewees state that no one monitors whether they are sitting in the office or doing their work from home or in a coffee shop, results and performance are ultimately what counts.

This does not mean that such respondents do not see the flipside of flexibility. These managers typically automatically synchronize their phones with their e-mail accounts so they can be notified of new e-mails at any time. Constant availability and connectivity results in late night e-mails, phone calls and work in the evenings or on weekends. This is strengthened by the fact that due to constant meetings during the day, operative work cannot be finished during working hours and managers have to take these tasks home. A common way of handling this situation is what may be labelled the 'night shift' and 'morning shift'. As mentioned, the managers often arrive home with tasks outstanding. If they opened their laptops immediately after arriving home this 
would steal the little time they have from their families. As a solution they put off doing work while having dinner, bathing their children and spending the evening together with them, and then after the children and partner have gone to sleep they continue to work. This can involve 1 or even 2 extra hours of work between $10 \mathrm{pm}$ and $1 \mathrm{am}$. With this strategy they do not have to sacrifice the precious time they spend with their children and wives, but it does reduce the time they have for sleeping or relaxing. Some interviewees found this method exhausting since they could not fall asleep easily with a busy mind. Those managers changed to using a morning shift which involved waking up earlier, before the family, and doing the same: finishing tasks, answering e-mails and so on. With this strategy their whole late afternoon and evening could be devoted to the family and sleeping peacefully.

In both cases work enters the home; the border between work and home is permeable. Interviewees who could be designated as 'integrators' seemed to be satisfied with this strategy. This does not mean that they always liked to work in the evenings although some consider such challenging work-related tasks a hobby - but they see it as a 'something for something' deal: they receive flexibility, freedom and work autonomy from the company in exchange for being available for work outside of regular working hours. As already mentioned one of the male group managers has a two-year-old son with a central nervous system disorder who needs constant care and attention. The perfect solution for him would be paid leave so he could remain with his son around the clock. This option is not available to him, and being the breadwinner of the family requires him to work even harder. In the given circumstances, however, he is very satisfied with the company's attitude towards him and treats his situation as a 'deal'. His boss tries to lighten the burden on his shoulders by allowing him flexibility in arranging his private matters during the workday, and in exchange he does not mind working late in the evening or being available all the time.

On the opposite end the scale are those who prefer to separate work and family. One third of respondents are satisfied with the way they can differentiate between the work and family domain. They admit that it is not easy, and they had to learn how to do it. Since these managers do not like to mix work and home, they rather choose to stay longer at work or to arrive earlier. During working hours or overtime they try to finish up their daily work since at home they do not want to open their laptops again. For many interviewees, the period of time spent travelling home after work serves as a transition space between work and home, not only physically but also mentally. Driving 
a car, or for some sitting on a bus helps them to compose their thoughts, make their final business calls and close the working part of the day. With the help of this ritual they change over to their home role and mood. Another very important characteristic of these managers is that they do not synchronize their phones with their e-mail account, or this function is not automatically engaged. Therefore they do not check their work e-mails all the time while at home, thereby stopping work from interfering with their family life. This automatically raises the question of whether their rejection of constant availability is resulting in them harming their careers. According to these interviewees who separate work and family life, such a lack of synchronization does not result in any negative consequences for their careers. One relevant difference, however, is that these managers do not prioritize work in the first place. They are engaged to the company, they like their jobs, but they also emphasize that work is not the most important thing in their lives. When remembering their past self, they usually refer to themselves as very ambitious and perfectionist employees. "You have to let things go and not give a shit. If you don't stand up at 5 pm, this work consumes you. I put in the amount of work which fits the salary I get for 8 hours' work. " (head of department, man, 41) But after reaching a certain level in their careers and establishing a family they do not chase success at all costs. They have learnt to say no, and that they do not need to make exceptional efforts all the time. They also had to realize that if they do not complete certain tasks, someone else will do them instead of them. This seems to be a difficult change in attitude since these employees still care about their work, but they have decided to change the priorities in their life. "I have lots of colleagues who don't understand how I can resist checking my phone on the weekend. (...) And funny or not, the biggest reason is when you have a private life which doesn't allow you to work constantly. If I didn't have a family and I were single I would surely work much more. But having children means that you can't really think of anything else." (head of department, man, 33)

Such separation of life spheres does not mean the complete rejection of flexible working opportunities. Those interviewees who consider their work and home distinct life spheres also use flexible working hours and home office from time to time. These few interviewees may be labelled 'alternators', although there is quite an overlap with this category and the separation type. Manager fathers in the sample who prefer a strategy of 'altering' also prefer to exclude work from family life. But they talk about the separation of tasks, not the separation of roles in the sense that they see themselves as integrated individuals: "When I'm home, I'm with my children. When I care for my 
children, then I care for my children, when [I care for] my wife, then for her. (...) But I think it's good when the family knows what am I doing, what my work is, what the difficulties and joys in my work are, when they know in advance that I have a big project and I can't come home early. (...) Complete separation is not healthy, rather on the level of tasks." (chief, man, 43)

Finally, one fifth of interviewees really seek to separate the two life spheres but have not been able to manage it yet. In that sense, these managers can be considered integrators, but not according to their free will, but rather due to the lack of control they have over blurring borders. They wish for the time when technology was not that advanced and that work could not be brought home. They want to cut back on the amount of work they do, but their perfectionism does not let them, while at the same time they also feel the external pressure to perform. These are the individuals who perceive low boundary management control, and face the most work-family conflict.

In addition to the typology given for the boundary management styles, other individual adaptive strategies can be identified. Half of the respondents - regardless of whether they are integrators, separators or alternators - emphasized the importance of consciousness, planning and prioritization. Even those who enjoy their permeable borders and flexibility are aware that flexibility is a double-edged sword. Therefore, they draw attention to the importance of using it appropriately. The problem might stem from the fact that nobody has taught them how to use this novelty correctly, to turn it into an opportunity instead of a disadvantage. Some directors perceive their own responsibility during this period of adaptation, highlighting that they have had to learn to deal with it, just like anyone else. Consequently, the conscious and clever usage of flexible opportunities and technology is necessary for turning it into an advantage in terms of work-family harmonization: "This is a very new problem, the internet, and the fact that we can take work home. (...) So we have to learn to incorporate these mechanisms into our world. If we don't, it will only lead to conflict. Not because anyone wants to cause harm, or because the devil has crawled into the house in the form of work, but because we don't have the tools to help us in these situations." (director, man, 40) For those who like to separate work and family, consciousness rather means the ability to define borders and clear priorities, and say no to certain tasks. This can be seen as self-defence against the disadvantages of flexibility (such as working even more hours or being available all the time). Besides this, it is a general remark from most of the interviewees that, after having a child, life becomes more planned and starts to lack 
spontaneous decision-making. In order to make the balance work, one has to think forward and plan the week ahead. This needs plenty of coordination and communication, especially with one's partner. The respondents highlighted the importance of evaluating whether an e-mail or work is urgent (and thus has to be dealt with) or can wait until tomorrow. The terms most commonly used by the interviewees were time management and prioritization. They find these two strategies indispensable for achieving a harmonic working style and family relations. It can be seen that this perspective is completely focused around the individual, emphasizing the individual's responsibility for work-life balance.

Finally, the last type of strategy involves accepting social support in managing the work-life balance. Support can take several forms: familial help (first of all, help from partners and secondly, grandparents, other relatives and friends) and paid help ( such as the services of babysitters or cleaners), but company support was also mentioned. When it comes to support in work-life balance, interviewees thought about their families firstly, family meaning their partner in most cases. Manager men in the sample usually divide responsibility for work along the traditional gender roles, especially when they have young children. In all cases their wives stay at home with their children, taking care of the household at the same time, while the men are the main breadwinners in the family. It seemed from the interviews that the wives accept this division of labour. It often transpired that the wives were working in similar professional positions before they took maternity leave. A few decided not to go back to the same sector after their maternity leave, but rather to choose something flexible and complementary to their husband's wage-earning activities. There was only one man in the sample whose wife occupied a higher position than him. In their case, the wife had gone back to work after 6 months, and they used the help of grandparents and a babysitter.

I also asked the interviewees how they support their partners. Some men seemed a bit confused by the question. Many of them admitted that they do not do an equal share of work at home. One of the group managers even feels that he has destroyed his wife's career. But to the question a wide range of answers were given, including the provision of emotional support, financial contributions, some share of housework or support for their wife's return to work, although others did not think that their wife needed any support. A few managers seemed confident about their own role and saw their main status as breadwinner as sufficient contribution to the family, while more of them tried 
to imagine themselves in their wife's position by emphasizing the importance of their careers as well. For most of the couples, however, the wife's career was regarded as secondary: "The enterprise is ours (...) a small carpet producer. It's rather a toy, it's not about the money, but it's there to produce, to produce value. And she [wife] is responsible for that (...) She told me in 2005 that she didn't want to go back to the bank to work, instead she wanted to play this role. And I celebrated the idea. So she is not sitting at home being a housewife and cooking stew, but she is occupied, she can express herself... so she feels useful." (director, man, 40)

Among family support, the role of grandparents was mentioned in second place. Around half of the respondents stated that they could rely on the help of their or their partner's parents, especially as concerns taking care of a child, and rarely with cooking and cleaning too. A major part of the respondents, however, can use this support only occasionally because the grandparents live far away. Two directors complained about the distance, explaining how important the role of grandparents is in the life of the children. In some cases, the interviewees did not want to rely on their parents too much because they are already old, suffering from illness or feeling weak. The work-family literature usually focuses on children when talking about family, although taking care of aging parents is a very relevant issue as well.

Outside of support from the family, paid service is also used as a work-life balance solution, although only 7 of the manager fathers in the sample employ a babysitter. Some men expressed negative opinions about hiring a babysitter. On the one hand, they found it expensive and luxurious; out of their league. On the other hand, they did not want to trust their child's education and care to a stranger. In contrast to this, there was one male group manager who was really fond of having a babysitter: "If you find a good babysitter with kindergarten-teacher qualification, then she knows 300 nursery rhymes, she knows skill-development games, what children like to eat, while I have to read 600 books and do a semi-professional exam on this basically. I noticed that my child is progressing at an incredible pace thanks to the babysitter." (group manager, man 29) The proportion who regularly hire a cleaner is very similar. These people are almost the same as those who employ a babysitter, but there are a few respondents who have a babysitter but not a cleaner and vice versa. As already mentioned, a few men had offered to pay for a cleaner for their wives, but their partners had rejected the proposal. This is a different situation to that with babysitting, where 
there did not seem to be any opposition within couples. Men who stated that they were set against hiring a babysitter were usually supported in their opinions by their wives.

As formerly described, the support of the family was primary for most of the interviewees, but around a third of manager men in the sample emphasized the importance of the workplace as well. Some of them mentioned it in first place, while others only after the family. According to these respondents, the company can help support the employees' work-life balance through its organizational culture, or through the activities of colleagues who work directly with them, like their teams or their supervisors. Besides thinking about family help, paid service and support from work, a fourth type of answer also appeared: one fifth of manager men said that they themselves were the core support of their own work-life balance. They stated their belief that they have to make their way through life primarily relying on their own strength, and talked about the importance of the already mentioned issue of consciousness in achieving a satisfying work-life balance.

\subsubsection{Female Managers and Work-Family Conflict}

The answers of female respondents in the sample were more similar to the male opinions than expected. Therefore most of the descriptions in the previous chapter are valid for the female managers of the company as well. Regarding their evaluation of their work-life balance, the female sample in the research showed more positive opinions: they were either satisfied with their current work-life balance or had mixed feelings, highlighting the fluctuating nature of their workloads. There were no women, however, who were very critical about balance in their lives. In relation to the definition of work-life balance, around half of them approached the term from the perspective of the family, expressing their desire to be able to take a child home from school, or to pay undivided attention to their children. They also more frequently mentioned concrete needs or events they wanted to participate in, while men tended to talk in more general terms of spending time with their families. Approaching the definition of work-life balance from the side of work, they described the same need to spend more time with children by finishing their tasks and duties within normal working hours, without overtime and worries. According to two female group managers, work-life balance could be easier achieved through the use of part-time work which left the women to better concentrate on their family duties and hold down a job. The rest of the managers 
defined balance in the classical way by referring to equal performance in both work and with the family.

Regarding the direction of work-family conflict, similarly to men, almost all women mentioned work-to-family conflict; namely, that their work influenced their family life more than the reverse. Some women also talked about the family's influence on work, but rather in the context of emergency situations like a child being sick, when family, of course, takes precedence - even more than in case of male respondents. Female interviewees, however, highlighted more strongly the fact that they do not like mixing private problems with work, and they expect the same attitude from their colleagues. This does not mean creating a formal atmosphere or a lack of amicable relations, but that work-related tasks should take priority during working hours: "As a leader and an employee who has worked here for a long time, I don't support the bringing-to-work of people's private lives. I survived a divorce, the loss of grandparents and a financial crisis without anyone knowing about it." (group manager, woman, 35) From all the 15 female interviews there was only one group manager who faced only family-to-work conflicts without experiencing any work-to-family conflict like the other interviewees did: "I can separate it [work] absolutely. Since I have had children, just a click [makes a clicking sound with her fingers]. - And in the reverse direction? - In reverse it's more difficult, especially after I returned. I left my 7-month-old baby at home." (group manager, woman, 39)

Women's perception of conflict sources was also very similar to manager men's experiences: time-based conflict with both physical and psychological components was the most frequent type of conflict female managers faced. This was followed by workto-family strain-based conflict, with women showing the same symptoms as men: exhaustion, sleep deprivation, lack of time and options for relaxation, impatience and even projections: "Yes, it happened once that I put my child in her clothes into the bath water because she didn't want to bathe. I tried to explain her for over 15 minutes that she should have undressed and jumped into the water, but when she didn't, then I said OK and... Of course I felt guilty.” (group manager, woman, 41) Proportionally, behaviour-based conflicts were more frequently encountered by women than by men. Although not every respondent found them a constant problem that affected their worklife balance, a majority of female managers were still able to mention examples of the traditional behaviour their environment expected from them. In a few cases, criticism came from their own families. One of the female group managers complained that her 
parents did not support her way of life, or the fact that she had got divorced since it was not in accordance with the morals of the community in the little town she grew up in. A female head of department in turn reported how she had had to convince her parents-inlaw to accept her career, and the fact that she was not a full-time mother. In the third example, criticism came directly from the interviewee's husband and children: "It's very interesting that when my husband has to travel, it's normal: OK, dad's left. But if I'm going out two or three times per week in the evening just to run, because that's when I have time, or to train or to meet my friends, then they [the children] ask for the second time "again?!". "You are always going out, mom!". I am the "always goingout mom" [she laughs]." (group manager, woman, 40) More common, however, is that criticism about mothers who continue their career-building activities after giving birth comes from the wider environment. Some female group managers described examples of receiving negative, malicious comments from other mothers at parent-teacher meetings. Only one group manager emphasized the gender differences in expectations within the company: "You are easily labelled an unreliable parent at the workplace. When a dad has to run off to his sick child, then wow, he is so careful and familyoriented. But if a mother runs off to see her sick child, then "you see, again, who knows, maybe she will come in with new nails or a new haircut tomorrow"..." (group manager, woman, 35) For the other female managers, expectations towards being a 'good mother' were not externally-driven, like in the previous examples, but rather internal. However, respondents added that they could not imagine their lives 'only' as full-time mothers. They like their jobs and often they feel more confident about their performance as managers than mothers. Sometimes this derives from the fear that as leaders at work they might be too strict at home with their children; consequently, they rather compensate for this by being too indulgent, and sometimes even inconsistent with their children, resulting in disciplinary problems.

The findings about the consequences of work-family conflict are very similar to those revealed through the interviews with men: a sense of guilt is one of the most frequent consequences, primarily due to the lack of time and attention spent on children, but in a few cases concerning the interviewee's partner. The sense of guilt also appears in connection with work, since most of the female managers in the sample are very ambitious perfectionists, always striving for maximum performance that often results in a time squeeze and stress as well. As already mentioned, there are more divorced women in the sample than divorced men. It cannot be concluded that this is due to their 
careers or a problematic work-family balance. Still, it may be assumed that it is more difficult to maintain a partnership in a society with a conservative gender context when a woman deviates from traditional gender roles. Those two managers who found new partners highlight the importance of the support they receive from their new partners. However, the situation was the same for married women, since they all emphasized the importance of the teamwork they have with their husband.

As for the strategies employed for balancing work and family, most of the female managers can be labelled integrators who enjoy the permeability of borders, especially with the help of flexible working options, which they consider a big help in reconciling work and family demands. Female respondents also highlight, however, the importance of consciousness and the wise use of technological devices. For example, when they talk with their children they try to avoid checking their phones every minute. They add, however, that they do not see it as problematic to answer some e-mails after children have gone to bed, or their husbands are at football training. Even those who do not synchronize their e-mails automatically can be labelled alternators since they also highlight the importance of flexible working hours and a home office, although they try to put down work when at home.

The use of support seems even more important in the case of women as regards organizing everyday logistics: seemingly more challenges arise for those couples in which both individuals aim to have careers. Almost all the female respondents named their husband or their life partner as their biggest source of support. This covers emotional support, but also instrumental support such as sharing household tasks; first of all, childcare duty: "There was a moment when we had to clarify that if it's a common decision that we want to live among these circumstances, then it's necessary that I build a career. Then it's also a decision from his [the husband's] side whether he can support this or not. (...) And fortunately, he could." (head of department, woman, 42) As already stated, those women who are divorced but are now cohabiting feel that they have real support from their new partners. With one exception, ex-husbands play a role in their children's lives. As one of the group managers said, she had to divorce before her ex-husband started taking real responsibility for their child. The role of grandparents was also crucial for female interviewees. In addition to partner's and grandparents' help, one of the group managers could rely on child-care related help from her sister and brother too, while another female group manager spoke of an 'adopted granny' from whom she receives lots of support. Paying for services occurred 
proportionally more frequently among female than male respondents. According to these female managers, they were forced to realize that they could not deal with everything, and thus need to outsource some tasks. As one of the group managers said, she could share the tasks with her husband but she considered the weekend too precious to waste time on housework, even if they did it together. A female head of department spoke of the price of maintaining a career as a woman. Since she returned from maternity leave after 6 months she had to employ a babysitter and a cleaner, which consumed a very big proportion of her salary. Based on rational calculations, it was not worth working, but she wanted to continue her career.

\subsubsection{Discussion of the First Research Question}

Analysis of this sample of manager men indicates that the topic of work-family conflict has relevance, confirming the statement that the harmonization of work and family life is not only an issue for woman any longer (Eräranta - Moisander, 2011; Halrynjo, 2009; Duyvendak - Stavenuiter, 2004). First of all, the interviewees took the topic seriously and did not underestimate its importance, even in the cases when they did not report serious work-family problems. Secondly, a majority of male respondents were concerned by some type of work-family conflict, occasionally or in the long run. Interestingly, those interviewees who were truly unsatisfied with their current workfamily balance were all men, while female respondents tended to talk about temporary difficulties. Relating to the managerial position, these results support the findings of the company's Employee Engagement Survey 2014, where all levels of management reported a higher level of work-family conflict and workload on average than regular staff members. This is in accordance with the empirical finding (Allard et al, 2007; Mayo et al., 2011; Geszler, 2014) that managers often experience a higher level of work-family conflict than other occupational groups, and should therefore be an important target of work-family analyses.

Although the term 'work-family conflict' was used as the basic concept of the research, in many cases other work-family interface phenomena emerged in the interviews and were more relevant for describing the situation. In the process of harmonizing work and family demands, conflict was not the only outcome but positive interactions also occurred: for instance, valuable social relations such as the emotional and instrumental support of the spouse proved to be acting as a positive mediator in reducing conflict, just as previous research has indicated (Kossek et al., 2012; Aycan - 
Eskin, 2005; Grzywacz - Marks, 2000). In the case of stress, the negative spillover approach is more suitable for describing the situation, since in most cases the tension stemming from the work domain spilled over to the family domain in the form of impatience, frustration or projection. Moreover, compensation theory may even be relevant since free-time activities and other methods of relaxation are used as tools for releasing job-related stress. And, most importantly, the border theory of Clark (2000) cannot be bypassed as it describes a flexible working environment. In connection with using the boundary management style, the concept is perfect for capturing the complexity of the work and family balance in a post-Fordist working context, since just because borders seem to be permeable, this does not necessary result in everyone preferring to integrate work and family domains. Moreover, the level of permeability might be perceived differently by individuals based on their agency, even within the same company. Therefore, even if we choose a work-family interface approach as our analytical concept, we should not exclude the possibility of finding evidence for other work-family interface theories, depending on the context of our research. Consequently Dén-Nagy's (2013) remark about the importance of the research context in choosing the appropriate work-family interface approach appears to be very relevant.

The answers given about what the work-life balance means to manager men also mirrored the variety of literature on work-life balance, and work-family balance definitions. Some imagined it as equal engagement in both work and family life, just as Greenhaus and Beutell (1985) define it. Few men highlighted a lack of conflict (Frone, 2003), while others had a 'components' approach (Grzywacz - Carlson, 2007; Greenhaus - Beutell, 1985), mostly emphasizing features of family life, like quality time with the family, or presence at home. Finally, even the overall appraisal approach (Clark, 2000) emerged as a focus on general well-being and the entirety of life. This finding might draw attention to the deficiency of using a single general question about work-life balance satisfaction in quantitative surveys that is unable to capture the diversity of meaning and complex nature of the phenomenon. For this reason it also seemed worth structuring the work-family conflict along the three types defined by Greenhaus and Beutell (1985). Although as Sebők (2015) highlights, time-based, behaviour-based and strain-based conflicts are not ideal types, interviewees' answers could be fitted into these categories and helped to structure the different dimensions and components of work and family harmonization. All three kinds of work-family conflict emerged from the interviews, although obviously time-based conflict was the most 
frequent and serious problem, just as described in work by Nagy (2008), Tóth (2007) and Primecz (et al., 2014), while strain-based and behaviour-based features raised problems as well that were not mentioned in the former empirical research. The direction of time-based and strain-based conflict was work-to-family in almost every case. Interestingly, it applied to female managers in the sample as well, although previous international findings state that family-to-work conflict frequently occurs among women (Greenhaus - Parasuraman, 1999; Hill et al., 2004). This is in accordance with Hochschild's (2001) findings that men and women have similar experiences regarding the harmonization of work and family life.

In connection to time-based conflict, psychological distractions are as important as actual overtime. As could be expected from previous empirical findings (Utasi, 2011; Blaskó, 2006; Szalma, 2014), the working hours of managers are long and include plenty of overtime. In addition, the traits of the post-Fordist time regime, like a feeling of general busyiness (Lewis et al., 2007), time pressure and a boundless working culture (Kvande 2009) or flexibility (Kvande 2009; Tausig - Fenwick 2001) all appeared in the interviews. Flexibility was a core component from the beginning, and emerged with both meanings: employee-driven and employer-driven flexibility (Hobson - Fahlén, 2009). The majority of manager men understood it to mean employee-driven flexibility which offers autonomy and a solution to the work-family balance, while others highlighted the disadvantageous way in which flexibility can lead to even more work and act as a tool by which companies can exploit employees. It is an important observation that while Primecz (et al., 2014) and Tóth (2007) found that men rarely used flexible working options to achieve a better work-family balance, and chose instead to separate work and family according to the traditional division of spheres, manager men is this sample reported that they tended to benefit from employee-driven flexibility. It is important to add that this it does not always mean the frequent use of flexible working hours and a home office, and does not include part-time work, but at least these options were not seen as initiatives only targeted at women. One feared consequence of time-based conflict is a lack of time to spend with family that leads to sense of guilt, like research by Nagy (2008). A shortage of time on weekdays required compensation on the weekends and 'quality time', just as Takács (2013) and Nagy (2008) found in their studies. Although in the research of Nagy (2008) manager men complained about having a lack of time primarily for children, at this company many of 
the fathers were so much focused on devoting enough time to children that it rather resulted in the neglect of their wives.

Behaviour-based conflict was the least frequently identified type of conflict among men; although it still concerned one third of interviewees in certain ways, it was rarely referred to as a serious problem. The findings completely confirm the results of Spéder (2011), Utasi (2011), Pongrácz and Molnár (2011) about the dual expectations men can face: they are expected to be the family breadwinners as in the traditional family form, but also to devote more care, emotion and time to their families, especially children, which is closer to the ideal of the involved father. These expectations are both external, (derived primarily from the wife and children) and internal (regarding the fathers' contradictory desires). Based on the above-mentioned national research, this phenomenon can be understood as a process of very slow estrangement from traditional gender attitudes and division of labour, although it is still very far from the modern understanding. Rather, it involves a mixture of both traditional and modern elements in attitudes and practises, similar to those described in international studies which relate to the complexity of fatherhood (Wall - Arnold, 2007; Rudberg - Nielsen, 2012).

Strain-based conflict appeared in different forms and at varying levels. First of all, respondents talked about job-related stress (Bell et al., 2012); namely the high workloads and pressure which spilled over to the family domain. This is not surprising, since as Mayo and her co-authors say (2011), competition-driven pressure has forced organizations to downsize and reduce hierarchical levels, adding complexity to managers' jobs. Consequently, managers not only face longer working hours but a high level of responsibility (Szalma, 2014). Moreover, Hungary is one of those countries where the highest levels of negative stress that stem from work are perceived (Utasi, 2011). The most general consequences were impatience, quarrels with wives, and projection of frustration on other family members. In addition, the lack of perceived control of boundary management was also associated with a higher level of stress, just as Kossek and Lautsch (2012) and others (Mellner et al., 2014; Mayo et al., 2014) have observed. Those manager men who wanted to separate work and home were least satisfied with their work-family balance but felt that the company did not support this, or that their own perfectionism did not let them since they did not want to lag behind. Only very few men reported to having serious levels of stress that were resulting in consequences for their health. Their main problem was the lack of hobbies or relaxing activities which could compensate for the effects of stress. As Utasi (2011) states, one 
reason for this is the culture of long working hours which leads to a lack of time and energy for hobbies and relaxation, but it might be also understood by managers not finding the appropriate creative activities to compensate for their feelings of stress. Similarly to Blaskó's (2006) findings, despite the time squeeze manager fathers at home do not report to having feelings of stress about household duties, and rarely feel that their family responsibilities are affecting their work performance; a finding which matches the traditional division of paid and unpaid labour.

The boundary management style concept was used to describe the adaptive strategies that manager fathers use to harmonize their work and family responsibilities. Although in most of the cases there was a clear preference for either separation or integration of work and home, as Kossek and Lautsch (2012) conclude, hybrid types of boundary management styles could be identified. First of all, complete separation of domains is hard to imagine since every manager had certain levels of permeability of borders through their use of information technology, or sometimes flexible working hours and home office options. Moreover, separation rather emerged at the level of tasks and time, but not between the managerial role and father role. As Kossek and Lautsch (2012) argue, the dualistic view of family-centred or work-centred preferences is a great simplification. Manager men usually felt engaged at their companies and found their careers to be important parts of their lives. Consequently, most of the men in the sample could be labelled dual-centric since they highlighted the importance of both family and work, although the proportion of involvement in the two domains varied across respondents. These results also confirm the observation of Kossek and Lautsch (2012) that it is not only personal preferences that formulate boundary management styles, but largely the corporate environment.

Besides the boundary management style, the division of paid and unpaid labour within the family greatly influences the strategy manager fathers use to balance their work and family commitments. Entirely in accordance with the findings of Nagy (2008), Primecz (et al., 2014) or Tóth (2007), men in this sample could completely rely on the support of their wives in achieving work-family balance. As also known from international research, spousal support is crucial in harmonizing work and family life (Kossek et al., 2012; Neff - Karney, 2005; Grzywacz - Marks, 2000). Manager men see their own contribution to their wife's well-being rather in the form of emotional support, while from their wife they can count both on instrumental and emotional support (Aycan - Eskin, 2005), just as Greenhaus and Parasuraman (1999) find. This is 
also in accordance with the general finding about the 'support gap'; namely, that men receive help more frequently (and more helpful forms of support) than women do (Neff - Karney, 2005). The majority of the wives of respondents can therefore be considered to be 'corporate wives' (Kanter, 1977) who create the supportive background for their husband's manager career. The results were in line with international comparative findings (Van der Lippe et al., 2006; Allard et al., 2007) that the traditional division of paid and unpaid labour matches more closely expectations about the ideal employee, thus resulting in a lower level of work-family conflict. In contrast, men with dual-earner relationships experience conflict more frequently (Ladge et al., 2014; Allard et al., 2007), except when their partner's occupation is flexible enough to leave them in charge of family responsibilities, or when the couple uses paid help like employing a babysitter or a cleaner. Unlike in the previous national research, however, some men in this sample had realized that the share of unpaid labour within their relationship was unequal and could even reflect on their own contribution self-critically. I find this to be important, since it matches more closely the findings of quantitative research (Pongrácz - Molnár, 2011; Harcsa, 2014) which have identified slow changes in attitudes.

\subsection{Second Research Question: Organizational support, Agency and Capabilities}

This chapter follows the logic of the second research question and focuses on the organizational contribution to managers' work-family balance, with a special focus on corporate culture and flexible working provisions such as flexible working hours and a home office. Moreover, the chapter also describes the agency and capabilities of managers to claim a work-family balance, and how this is restricted by the compulsion to visibility and presence. The chapter, just as the previous one, ends with a discussion of the findings.

\subsubsection{Corporate Work-Life Balance Policy}

Regarding the company's work-life balance policy, the general impression is positive. Various initiatives are mentioned by the interviewees that are designed to improve employee satisfaction and engagement with the company: flexible working provisions, organizational culture, the support of supervisors, infrastructure, support for the return of mothers, a company bus, corporate events and a 'cafeteria system' (a benefits package). Since the flexible working options (namely, flexible working hours 
and a home office), and a supportive organizational culture were the most frequently mentioned elements of work-life balance policy, these initiatives are described in detail in separate sub-chapters.

In addition to flexibility and organizational culture, the support of a direct supervisor was another important factor, although this was not considered to be an official initiative but an informal one. Many respondents associate flexible arrangements with the attitudes of their bosses, stating that formal opportunities would be useless without the supportive and emphatic approach of their supervisors. Respondents often feel grateful that they can count on the humane attitudes of their bosses and that they do not face difficulties with using flexible opportunities. According to the interviewees, most of them have a good relationship with their bosses, sometimes even considering them to be role models. Only rarely did interviewees complain about their direct supervisors, and even in these cases they referred to former experiences at the company, not to the current situation.

Around one quarter of the interviewees said that infrastructure was something that made their lives more comfortable. The building has spacious offices, plenty of windows to let light in and a relaxing, pleasant and modern atmosphere. One group manager likened the venue to a sanatorium, or 'The Black Forest Clinic'. A canteen and a buffet with a comfortable coffee corner exist, providing both the opportunity for social interactions and saving the time of employees who would otherwise go out for lunch. A gym and a sauna can be found in the basement of the building. Some managers use these relaxation facilities and enjoy the fact that they are able to do their weekly sports activities within house, but most of the respondents see these facilities as nice options to have, but they rarely use them themselves. Only one head of department and a director had negative opinions about the gym, the former believing that a workplace does not have to be "fun", ("that is what one's private life is for"), while the director saw the gym as just one more way to spend even more time at the workplace. He even adds that one cannot even do sport without being disturbed by colleagues about work-related issues.

A few manager men mentioned support for the return of mothers to the workplace as part of work-life balance policies. Based on the answers of female managers, however, the picture is more complex. Most of them were easily able to return to work, but usually not to the same position they had before. One of the reasons for this is that managerial positions are not usually available on a part-time basis. The 
other reason is that structural changes were occurring so it was not rare that whole groups or divisions were differently structured by the time the female managers returned to work. Consequently, new positions had to be found for them.

Furthermore, three other initiatives were mentioned sporadically: the company bus, corporate events and the cafeteria. The company bus offers frequent rides in the morning and in the afternoon (from $2 \mathrm{pm}$ ) to several locations. Some of the interviewees praised this facility, emphasizing that it is very rare to have such a frequent and well organized corporate bus service. Somewhat contradictorily, these managers all travelled by car, while those respondents who used the company bus had more complaints. Appraisal of corporate events was also divided. A few respondents stated that they enjoyed family events because they could take advantage of the opportunity to introduce the company to their families. This decreased the 'distance' between work and home since their partner could get to know the environment in which they work, and give them the chance to meet colleagues. Through this the family could gain better insight into what the managers are doing. Such events seem to be of high quality, and include concerts, programs for children, sports, games and a barbecue, which is great entertainment for children. However, two respondents stated that these are only extra diversions which steal time from their private lives. Only five respondents regarded the cafeteria system and bonuses as a work-life balance benefit.

And finally, some male managers were critical of the company's work-life balance policy, doubting its family-friendliness. According to these individuals, flexible working opportunities are 'nice thoughts', but as long as the pressure on the company in the private sector is huge and competition remains intense, these policies stay at the surface and are not real solutions to such problems. They are designed to cure symptoms, not causes: "As for me, I believe that flexibility and a home office are communication things rather than useful tools. (...)I will do this work only while it's worth it." (head of department, man, 39) Other men formulated it more coarsely; the company's family-friendliness is "bullshit", "rhetoric" and "exists only on paper".

\subsubsection{Home Office}

Next to the flexible use of working hours, the home office is the second most popular work-life balance tool that employees benefit from. There are several reasons for choosing to work from home. First of all, when a child is sick the father may have to stay at home. This situation primarily occurred with those men whose wives were also 
working, so they had to manage these situations together. Interviewees find the home office to be a perfect solution to this problem since they can take care of their child but do not need to be on leave. Summer holidays from school are real logistical challenges for dual-earner couples since parents have to organize their holidays and leave in a way that someone can be with the children at home. Some of the managers use a home office when they themselves feel sick. This, however, is prohibited by corporate regulations since in the case of illness employees should go on sick leave. Nonetheless, many of the managers use a home office in the case that they are not seriously ill; for example, they only have a cold. In this way they can carry out their work in their home environment without the risk of infecting others.

The second most frequent reason for use of the home office is strongly connected to the culture of meetings and the open office system. Due to frequent meetings and constant background noise in the office, many managers face the problem of falling behind with their operative and administrative tasks due to a lack of time and quiet. One quarter of manager fathers reported that they or their colleagues used a home office to catch up with tasks, since at home they are not bothered by meetings or by their colleagues asking questions. Some tasks require the kind of concentration and tranquillity that is not found by everyone within the office walls: "If you use a home office because you simply need silence to work, because in a big open office thing you can't, because the level of noise is so high around you, then maybe we [as leaders of the company] are not doing it right. (...) If our company could create a place in this environment where you could spend 2 hours in silence ${ }^{16}$..." (director, man, 40)

A few interviewees also stated that administrative and home tasks were reasons for using a home office, such as having the chimney swept or the gas meter read, or arranging issues at offices or local authorities which are usually closed by the time the managers' workdays end. And, finally, a few respondents live some distance from the company and spend hours commuting. In their eyes a home office represents the opportunity to save time travelling.

Around half of the respondents reported to having these positive experiences regarding the home office. Not everyone, however, is a supporter. One third of respondents do not find a home office efficient because it is not inspiring enough, and

\footnotetext{
${ }^{16}$ According to the HR assistant who was my main contact, the company formerly had a library where phone use and conversations were not allowed and employees could work in peace without any disturbances. The library, however, was not really used by managers for this purpose.
} 
they find it challenging to concentrate. There are too many distractions like the television, the postman, phone calls or family members themselves, especially children. The concept of a home office is difficult to explain to children since they do not understand why their father is working when he is at home and they demand their parent's attention. In addition, it makes the fathers feel guilty when they have to reject their child's need to be with them: "Many of my colleagues decide not to come in, because they can save time on the way back, and say how good it is to work from home. I can't. Exactly because I feel that the border between home and work will be blurred even more, and I can't handle this. And when my children are home, they can't handle it either." (director, man, 40) A group manager man explained that since he draws distinct borders between home and work, he finds it very difficult to use the home domain for matters other than private life. Two other male managers stated their reasoning by saying that humans are social creatures and thus they need daily interaction with colleagues. Sitting home alone and working in an isolated way is not something they are comfortable with. Some other manager men had found a third solution for home office problems: they do not work from home but find a neutral place like a McDonald's restaurant or a coffee house near their home. With this method they can enjoy the advantages of a home office like reducing the time spent commuting or not being distracted by meetings, but avoid the mixing of home and work domains at the same time.

Regardless of who prefers a home office, it is also a question whether such an option is available to everyone. In theory all managers have the right to work a few days from home. The practice, however, is different: the often-mentioned culture of meetings regularly prevents managers from benefitting from a home office. Meetings require a personal presence which makes working from home impossible. Although the technology is available for online meetings, a real presence is preferred and expected. More interestingly, the building in which the employees work was originally designed with the concept of a home office: there are fewer places in the office than the number of employees in the assumption that there will be always a certain number of employees working from home at any time. What is true is that the so-called floor managers who live in other cities far from the company truly have the alternative of working from home and are required to visit the main office only once or twice a week. Among the other managers, even regular home office users do not take advantage of this option more than 2-3 times per month since direct availability is still a determining feature of 
the workplace. Moreover in certain divisions such as project management or customer service, use of a home office is even more infrequent since employees in these fields always have to be available and ready to act.

In addition, not every manager supports the use of a home office, although they are in minority with their opinions. Their objection towards the home office is that they consider it 'a holiday'. They do not believe that users really work at home, therefore in their eyes use of the home office is essentially equivalent to paid leave for lazy employees. Only very few managers share this view, although one of them is a chief consequently, it must be assumed that this opinion has an effect on many employees' opportunity to use a home office. "There were cases when those who went home at 7 pm received acknowledgment. It didn't matter that he was playing on his computer from 4 to 7. He really was playing. But he was the example, because he stayed so long." (group manager, man, 43) Other interviewees, in contrast, explained why the home office was inherently liable to stimulate more work: the basis of this phenomenon is trust. Ambitious employees who want to prove themselves trustworthy will work even harder at home to show their commitment: "It took many years to develop a general corporate culture regarding this issue. Before this time, the company was really afraid because they were unsure how and to what extent employees would misuse this [home office]. I think that after that many years they realized that the institute of the home office inherently motivates work." (group manager, man, 36) Due to this mechanism it is worth the company sending people home to work. The phenomenon of the role model is very strong in the case of the home office. Managers who feel that they are trusted by their superiors try to propagate this example amongst their own team members.

\subsubsection{Corporate Culture}

Many of the interviewees praised the company's general culture. The three most popular characteristics of the company are its people-oriented approach, its informal communication and its democratic style of decision making. By people-oriented approach the respondents mean the respect the leaders evince towards employees of all levels. “... they don't judge someone by "wow, look that fat guy, what does he look like! - and he's bald!', which can easily happen at other companies. I've worked at 3 big multinational companies and when you are labelled like this... then I'm not interested anymore. Then you don't feel any engagement anymore. Yes, I have been in such situations." (director, man, 40) As one director stated, this culture treats employees as 
autonomous adults who are trusted to act responsibly. Consequently, they are given plenty of scope to organize their work as they wish. He also adds that a company is not a faceless entity, but consists of people who make it work. Therefore what the leaders convey is highly important.

The company has a flat structure, so interviewees find that the hierarchy is less dominant. The opinion about the informal way of communication within the company is very positive. The general agreement is to use informal forms of address both in written and oral communication to avoid circumstantiality, and to make everyone feel equal. I experienced this myself when receiving e-mails and in personal meetings as well. The open office system, however, is not enjoyed by everyone because of the constant noise and lack of isolation and tranquillity. On the other hand, interviewees find it positive that in this spatial structure everyone is approachable, even the top leaders. No one has their own, separate office, not even the CEO, which strengthens the sense of equality and makes communication easier, helping to eliminate many bureaucratic obstacles. It is possible to joke with top leaders, relations are not distant or cold due to the existence of a rigid hierarchy but respect can still be maintained between employees. Most of the managers feel that in this culture the atmosphere is very open and indirect which relieves frustration.

The democratic way of company functioning, however, is more divisive. By 'democracy' the interviewees are referring to the decision making procedure whereby everyone's idea is listened to and taken into account based on the concept of equality, as well as the absence of the direct rejection of any suggestions. Around half of the manager men see the positive side of this approach, since every idea can be tested, and everyone's word counts. The opposition to this approach is that it is partly the reason for the overload they experience. Since the company has too much ideas and launches too many projects in parallel, the workload is very high. As a group manager reported, the company loses focus with this attitude. Another problem is that since this democratic approach avoids direct confrontations and direct rejections, manager men often feel that no decisions or no strong decisions can ultimately be made. It can be often confusing when managers feel that they have been left without feedback and concrete guidelines. One of the leaders explains that such a lack of clear decisionmaking might be the result of an over-extended collectivism and the delegation of decision-making rights to lower levels. On the one hand he supports the principle of collectivism, but he highlights that it should not hinder efficiency and fast decision 
making on the other. The other leader sees it differently and explains the vague decision making procedures by a lack of responsibility taking. He believes that many people do not dare to back their ideas and therefore they try to avoid negative consequences by taking an indirect and unclear approach: "The other problem in the culture is that we spend incredible amounts of time discussing and challenging each other's ideas about how to solve a task. We put more energy into finding out why an idea would not work. So everyone wants to defend himself from every direction, and pointless politics take place for months about a 2-day job [laughs]." (chief, man, 49) A few managers, however, defended the approach to democratic decision making, stating that just because it uses a respectful and polite tone, it does not mean that decisions are not taken. A director explains that despite the appearance, this is a demanding culture, and regardless of whether a request is formulated in the guise of "I highly recommend that...", this still represents a command.

Basically, all which is described by the informal and democratic terms is what the interviewees call Scandinavian culture. Most managers feel the difference of being under Scandinavian ownership, especially those who have more experience with other companies. The evaluation of the Scandinavian management approach is unambiguously positive and is seen as an example to follow. Critiques are therefore not aimed at Scandinavian culture - except for the lack of clear or direct feedback - but rather at the inadequate adaption of Hungarians to this foreign culture. Some managers admit that there were misunderstandings due to the different methods of communication between the two cultures. Hungarian leaders had to learn to understand the signs and the attitudes that the Scandinavians represent. One director described the phenomenon whereby the principle of seniority was so strong among Hungarian employees that they interpreted every word of the Scandinavian CEO as a command, whereas he was only sharing ideas and did not expect them to become projects: "I think we, ourselves, put into operation the perception that we should turn to the leadership team with almost everything. This has many components: first, everyone wants to have visibility and take on topics which are not even relevant. Second, our leadership team is very inquiring and our CEO is a truly visionary person who is very open (...) it started around one year ago that the CEO, whenever he meets someone, starts by telling them that it's only an idea, he doesn't want it to be taken seriously, that the idea should be placed where it belongs on the list of priorities. Before this, projects were made of this [ideas], priorities were made, legions were made because the boss said that's what we have to 
do." (director, man, 40) The open office and the clear desk system - although these are found not only in Scandinavian culture but in American corporations as well, as one of the managers clarified - was also difficult to get used to for many managers. Although having a clear desk means that no one has a fixed place and therefore no one should leave personal belongings on the desk, the practice deviates from the original concept. Hungarian employees usually insist on going to their habitual places, and if they are not occupied then they tend to choose always the same one.

Although interviewees feel the positive influence of Scandinavian culture they claim that the company is still very far from having Scandinavian standards. As an example of this, they talk about their experiences abroad when they had the opportunity to visit the parent company. Interestingly, the one manager from the sample who is currently living in Denmark with his family does not see the case negatively. He perceived that there was more family friendliness from the Danish company, for example in the way that everyone left work around $5 \mathrm{pm}$, but he attributed this to the general gap in attitudes between the two societies. Others mentioned that in Scandinavia employees might have more weeks of vacation without being disturbed, while here they are happy if they can go for one or a maximum of two weeks and then are still expected to follow company issues.

The often mentioned meeting culture is also criticized, but rather from the perspective of the characteristics of Hungarians. Some managers have seen that the parent companies only organize meetings between 9 am and 3 pm taking into account parents' schedules. At the Hungarian subsidiary, however, this interval is more extended and it is possible that meetings are even held even at $5 \mathrm{pm}^{17}$. "I tried many times to cut back on the number of meetings, skipping some, but the organization resents it. It's a very interesting thing that in this culture delegation is not accepted." (head of department, man, 35) The calendar of every manager, especially those occupying higher positions, is very busy and might be full of meetings from 8 am to 6 pm, even two weeks in advance. Since the interviews were organized using Outlook Calendar I obtained insight into the schedule of managers. As the previous quote shows, it is very difficult to change a meeting date since one has to adapt to the schedule of many others. This organization and the coordination needed to find a free spot in another person's calendar causes frustration for many managers.

\footnotetext{
${ }^{17}$ According to the HR assistant, there were attempts to create 'meeting free hours' but in the long term employees tended to occupy those periods with meetings as well.
} 
It can be assumed that the democratic concept of decision making and personal presence are correlated. If everyone's opinion is to be taken into account, it logically requires the presence of every actor. Delegation is seen as one possible solution, but not everyone is willing to hand over decision-making rights to others, while those who would gladly empower their subordinates do not always have this option since in many cases they do not have a deputy. As can be seen, there are contradictory perceptions regarding empowerment. Some feel that the company is decentralized and every decision is delegated to include lower levels of management, which is what makes the decision making procedures circumstantial and meetings slow. A few heads of department and directors have the perception, however, that the company is still centralized in the sense that decisions are made by higher level leaders (by the interviewees), and they cannot delegate tasks. Consequently, all the burdens which stem from a lack of empowerment are on their shoulders. A male head of department summarizes the situation by saying that the company supports using a home office on the one hand, but is a leading firm in Hungary when it comes to organizing meetings at the same time. These two factors also correlate in the sense that it is partly due to the meeting culture that no time remains for other work than decision making. Higher-level leaders see as well that a lack of human resources and the inappropriate organization of meetings cause tensions and might even be affecting performance: "We have better indicators than the competition. And we have higher income and profit. Staff efficiency has a very positive message on the one hand, but on the other hand I'm very critical, because we strongly exploit people." (director, man, 42) Consequently, even if a home office and flexibility are both formally and informally supported, the company's everyday functioning and the organization of work can restrict the agency to take advantage of flexible opportunities.

A small piece of research was undertaken about the culture of meetings by the HR assistant who presented to me the results of the Employment Engagement Survey. The HR Department had become aware of the controversial outcomes of too many meetings and wanted to map the diverse root causes which were leading to this inefficient meeting culture. According to this survey, $47 \%$ of employees' working hours were occupied with meetings. They list the following causes of the inefficiency of meetings: meetings serve for the purposes of alignment; behaviour is driven by example; limited time is available for discussions; the home office is not supported at a few departments; there are no clear rules about who has authority for decision making; 
uneven distribution of workload; low respect for meeting/email norms; decision-making authority is positioned at a high level; high number of initiatives. As can be seen, these causes are in accordance with the findings of interviews summarized so far. The HR Department intends to develop the process of work by increasing the efficiency of meetings, by upgrading meeting rooms and by supporting new ways of working, for example, by using agile or service-design processes.

All in all, the interviewees do not see the Hungarian subsidiary as a Scandinavian company but rather as a mixture of Hungarian and Scandinavian cultures where the Hungarian employees still have a lot to do to catch up to Scandinavian standards. As some interviewees reported, the Hungarians have adapted to the pleasant elements of the Scandinavian culture and merged it with their own attitudes: "In Scandinavian countries this culture developed in the last decades and people know how exactly to manage. They start the morning with the gym, they get up early and arrive at work early but at half past 5 they leave, whatever happens. They live in an incredibly structured way. While in Eastern Europe the normal culture of work was ruined under socialism, and after the 90s this new world burst into our life, and my generation had no-one to learn from about the working culture of this business life. Typically, we work in a less structured way, more ad hoc. Our daily agenda is not managed, we are swimming in work, we stay late therefore we are tired the next day and we don't go to the gym... and the spiral continues." (chief, man, 43) The interviewees always add, however, that compared to other companies that operate in Hungary (regardless of whether Hungarian or foreign owned), this company is still a much better place to work at. Many managers in the sample have been working at the company for more than 10 years and there are some who returned after spending some years at competitors. These interviewees explain that, despite all hardships, they have their reasons for staying and would not continue to work here if they were generally not satisfied. Those who have worked at other firms usually claim that these other workplaces were much more unsatisfying. Even the public sector does not help with the harmonization of work and family life as much as may be assumed. Most of the interviewees accept that the competition is very intense in the private sector and that the company has to adapt. But the majority of the managers appreciate that, despite the fast pace and pressure of the workload, they at least have more autonomy and flexibility than employees at other companies. In conclusion, the interviewees in general look at the Scandinavian situation 
as a dream that is out of their reach, but within the Hungarian labour market context they are more or less satisfied with the company.

\subsubsection{Visibility and Flexibility Stigma}

Flexibility and the blurring borders between work and home have changed the original nature of working culture and have brought new challenges into employees' lives. Many interviewees see the flipside of flexibility: on the one hand it gives freedom and an opportunity to reschedule one's day, while on the other it extends the borders of working time. As described in the literature, we can talk about flexibility in two ways: employer-driven flexibility and employee-driven flexibility (Hobson et al., 2011). What employees enjoy about flexible working hours and the home office has already been summarized, but why is flexibility a good deal from the company's perspective? Technology makes it possible for employees to reach work from anywhere, and be available all day. The company makes a profit from this since employees can now spend more than 8 hours doing work: "It is very useful that [there] is no card-punch and we don't need to come in like that. Although I think that it is more useful for the company than me. So the company gives us flexibility, but most probably due to the flexibility I'm putting more into it voluntarily than I should." (head of department, man, 39) It is also difficult to say what counts as work. Quickly checking e-mails at home does not seem to be an exhausting task, but it still prevents an individual from quitting the work domain and remaining involved in the home sphere. As previously explained, blurring borders do not necessarily represent a problem for everyone, and there are many managers who enjoy the lack of clear distinction between work and home. They do not mind writing e-mails at $11 \mathrm{pm}$ or receiving phone calls from colleagues during vacations. But the real question is whether this attitude has become a norm, an unspoken expectation that employees should always be available even after working hours. In other words: is visibility required? The answer is not clear. Those managers who were described as integrators in terms of their boundary management style are rather internally driven employees who do not consider visibility to be a form of external pressure but a component of internal motivation. In contrast, those interviewees who prefer to attempt to separate work and home but have not been able to accomplish this yet consider visibility to be required by the company.

Internally driven managers are aware that they exceed the normal amount of work and put more energy into it than is expected. Since they feel motivated to do their 
best they do not blame the company for the workload. They admit they are constantly available and they even see the disadvantages of this, but they do not consider their behaviour to be a response to the external expectations of the company. As they say, they could switch off their e-mail synchronization system but they do not want to. They are available because they choose to be available, since it gives them a sense of security and control. They follow corporate news even from home by checking e-mails, which makes them up-to-date and gives them confidence. They consider the fear of lagging behind or being left out of an important decision a real threat. Consequently, their motivation is derived from three elements: perfectionism, addiction and the need for control.

Giving the perfectionism of these internally driven managers, they always strive to do a perfect job. Since deadlines are short but multiple projects are running simultaneously tasks cannot be done within regular working hours. "You can observe through watching these colleagues that when they have a week in which they have been able to finish their job in 8 hours and could go home, they instead take on more tasks because there are always plenty of ideas and things to improve. It's another question if it is because of this habit that these colleagues are successful in their jobs and that's why their careers are booming." (chief, man, 43) Some managers find their own perfectionism bothersome and are trying to cut back since they are aware of their own role in generating more work. Not everyone perceives this as a problem though: a few managers state that when the task is inspiring it is not gruelling to do some extra research on the topic or further perfect a presentation.

Regarding the constant e-mail checking, some interviewees admitted that it could be considered an addiction. They feel frustrated when they do not know the details of a situation and their curiosity drives them to read their messages all the time. As a male group manager explained, he read the mails in his corporate mailbox just as if it were his private mail folder, but he believes this is the default behaviour of his generation. "When we first got phones which you could synchronize e-mail with, people became e-mail addicted. And it was simply too difficult to stop; you checked all the time. When you received an e-mail, you answered. It was a bit like a gambling addiction, or similar to those who spend lots of time on Facebook or online." (chief, man, 43)

Since many employees follow this practice it can easily happen that important decisions are made online outside working hours. Consequently, those who want to 
participate in these processes have to follow events online. It seems, based on the interviews, that no one is called to account for not being available during these late night conversations but one has to be prepared if they are absent that others will arrive at a conclusion without them. Therefore it depends on the individual whether they will join this 'game' or not. Those who want to control processes cannot accept being left out from something. They only feel secure when they always have the necessary information and can influence events, even if they are on vacation, for instance. As a few managers stated, checking e-mails occasionally during a holiday makes them reassured that everything is going well and that nothing has gone out of control. Sometimes this feeling of security that control gives them overrides the potential inconvenience of work entering the private sphere.

Those managers who use a boundary management style of separation do not think either that visibility is required by the company or supervisors. They represent the philosophy that tomorrow is another day, and nothing will happen if they answer an email the following morning. This is connected to the ability to say no and draw limits on work. They accept that they might be left out from some decisions. It took them time for them to arrive at this acceptance since at the beginning of their careers they also wanted to have a say in every process. But after changing priorities in their lives, they learnt to withdraw from certain decisions, although they see that many of their colleagues cannot quit this habit. According to these individuals, their colleagues generate work for themselves and misinterpret expectations by believing that all of their work is required, while in reality most leaders do not expect anyone to answer e-mails at $11 \mathrm{pm}$. One of the chiefs and a group manager even add that they find it positive to be left out from the unnecessary discussions and receive only the conclusion. It was also mentioned that personal presence does not necessarily mean good performance. Accordingly there are high level leaders who are hardly visible but everyone can feel their influence, while some employees sit the whole day in the office to show their commitment, but are not effective. "You might sulk, of course, if a meeting didn't work out as you had wished (...) But you don't always have to be part of that [decision]. There are battles you have to fight, and there are battles you don't. You have to define your priorities." (group manager, man, 29)

A quarter of manager men believe, however, that visibility and constant availability are expected by the company, even if implicitly. These are the same interviewees who have a preference for separating work and family but do not feel that 
they are capable of this. A few interviewees talked about the need of their presence as a leader. They believe that, as leaders of a group, they have to be available for their subordinates. Two male managers also added that if they did not oversee the work of their colleagues, they would not be working efficiently. These interviewees would gladly bring back the old times when technology was not as developed and work could not be performed at home. They emphasize the negative consequences of technological progress and see flexibility rather as a tool the company can use to force people to work harder. They are frustrated by their phone beeping and showing new messages which causes work to intervene with their private life. But they do not feel that they are in a position to neglect or refuse this way of working because they perceive they are under pressure to conform. 'Unfortunately, these technical achievements don't help our work but make it harder. At the directorate or at customer service top management really expected people to be available 0-24. And not the current customer service director, but the previous one, well, she was extreme; she would call the heads of department on Saturday at midnight without a problem. (...) So, unfortunately, even if we have a flag outside saying that this is a family friendly company, it doesn't work above group manager level. This flag is bullshit." (group manager, man, 43)

All in all, the interviews suggest that visibility is not required at all costs, in the sense that moderate use of flexibility and the home office will not cause the user to be punished. Employee-driven flexibility (meaning work autonomy) is a real option which most managers benefit from. The scale of its use is the question: due to meetings (namely due to the need for personal presence) the extent of flexible opportunities is restricted and is narrower than many of manager men desire. In addition, employees in certain fields such as customer service and property management face a disadvantage since their availability is more crucial in these domains. Flexible working hours and use of a home office, however, do not cause flexibility stigma, since except for two examples, the interviewees did not feel that there were any negative consequences from using these options from time to time. No managers are called to account for missing a late online discussion, although it might affect the career progress of ambitious employees. Consequently, without strict corporate guidelines online availability is strengthened informally by internally motivated people and might cause others to be drawn into using this routine who are afraid of lagging behind. Informal norms and the example of direct supervisors strongly define subordinates' room to manoeuvre. Therefore it is extremely important whether leaders are aware of their own behaviour 
and its influence on others. The agency and capabilities of managers, as well as the responsibility for work-family balance, is described in the next sub-chapter.

\subsubsection{Agency and Responsibility in Work-Family Balance}

Respondents were not directly asked about who they found responsible for their work-life balance but it transpired during most of the interviews in any case. Besides the role of the individual and the company, the state's influence on work-life balance was only mentioned once by a male manager. He highlighted the difficult situation of mothers in the Hungarian labour market. But apart from this case, the interviewees focused on describing the individual and corporate level.

The majority of interviewees see work-life balance as their own responsibility. They believe that they cannot expect the solution to their problems to come from the company. The company's aim is to make a profit and it is under constant pressure to do this. Therefore it would be naïve to expect the company to consider the employees' work-life balance as a top priority. The company offers a frame with tools like use of a home office and flexible working hours, but it depends on the individual how they benefit from these opportunities. As a group manager man summarized it, nobody tells him to go home otherwise his work-life balance will upset, but nobody forces him to stay. Consequently, as responsible adult individuals, everyone is provided with the autonomy to define their own priorities and act accordingly. In some cases, this means refusing to accept certain tasks, switching off e-mail synchronization or leaving work on time. But this requires self-awareness, and knowledge of where to draw the limits. Since in this sector tasks are infinite, one can never deal with all the unsolvable problems that exist and the company does not prevent employees from taking on more duties. According to a few male respondents, if the conditions are no longer compatible with their work-life balance needs, they will look for another job since it is they alone who can change their own situations. Even those interviewees who were critical towards the company are aware that employees who are attracted to these firms tend to work too much like 'birds of a feather'. Some managers add that many employees tend to expect to find the solution from others rather than themselves. This results in employees blaming the company for their own work-life balance problems, while it is them who could change their own situations. It is particularly Human Resource Department leaders that must face the expectations of employees that they should find solutions to their work-life balance. The chief of the Human Resources Department estimated that 
$80 \%$ of employees expect the company to solve their work-life balance issues. "Everyone is expecting us to create a new culture of meetings, or to tell them that everyone has to go home at 5. We can try, but life is not like this, only you can draw the lines regarding what work to undertake and what not. By the way, I wouldn't like to be told not to work at 6, because maybe it's important for me to finish a task." (head of department, man, 40)

Significantly fewer manager men considered work-life balance as a company responsibility. These interviewees were also aware of their own role in their work-life balance but they also alluded to the company's or their leader's part in this, often using a critical tone. "One thing is what I can control, like when I go home, how much I can forget about work at home and so on. But the other side is how much your boss and your colleagues respect this. Or how accepted it is that you don't pick up your phone during holidays, or you don't check your e-mails. I have very mixed experiences within the company." (head of department, man, 33) One of the chiefs was very critical regarding the company's functioning, although he highlighted the positive side at the same time. He believes he has to be critical, even self-critical, for the sake of improvement as he sees his own responsibility as a leader and as a part of the corporate culture. Some other managers also talked about the importance of informal relations in work-life balance. As already mentioned, those who have a tolerant and permissive boss try to show the same attitudes towards their colleagues. Consequently, if the top leaders of the company respect the work-life balance of employees, this attitude trickles down to the lower levels and becomes part of corporate culture. Therefore leaders through their behaviour are responsible not only for their own work-life balance, but for their colleagues' as well.

The approach to the responsibility question can be also captured by observing what the interviewees said they would change to achieve a better work-life balance. When I asked them this question at the beginning of the interview almost all the managers thought about the personal changes they could make. There had two components: what the managers would change in their private lives, and what change would they expect regarding work.

Answers relating to private change were most common, and included the improvement of planning skills, consciousness and prioritization. This suggests that most of the respondents take the working environment as a given, and do not feel they have much influence on working processes or corporate culture. They can only try to 
change their own attitudes or efficiency in order to perform in the expected way in every life domain. Some would like to let go of their perfectionism, while others have developed their own strategies such as the 'night shift' or 'morning shift' as a reaction to their work-family conflict. This does not decrease workloads however, but only help to structure it. A few interviewees would like to add sports into their daily routines and try to restructure their agendas accordingly. Two managers in the sample had decided to move closer to the workplace to save time commuting. These are major alterations to the private lives of individuals for the purpose of creating a better work-life balance and reducing time-based conflict. Besides these answers, there were two managers who had left their previous workplace because they could not maintain a satisfying work-life balance according to what was expected of them.

Only some of the interviewees were trying to change their working conditions, like one of the head of departments who intended to cut back on the number of meetings he attended, but as he said, the company did not support this. Another man told the story of how he was responsible for many more clients than was realistic and therefore had complained to his supervisors. Since his bosses had realized that the high workload was affecting employees' performance negatively they hired more people for that group, easing the pressure on the individuals. This is a rare example of when a manager acted within the company framework to improve his work-life balance and make a positive change. In most cases, however, interviewees accepted the conditions and seemingly did not feel that they had agency in this regard. Group managers in particular consider themselves to be insignificant in the frame of company operations, placing themselves at the bottom of the hierarchy of managers and acting according to this perception. This not only means that they perceive a lack of agency but that they also do not think much about their own influence on their colleagues. "We have talked with the CEO about how it looks when he works on the weekend and sends e-mails or calls me about some question (...) With one single e-mail he drags in 4-5 people. (...) Since we agreed on this 2.5 years ago he hasn't written and we haven't needed to work on the weekend." (chief, man, 43) Interestingly, only higher-level manager men, directors and chiefs appeared to be thinking about their own responsibility for other employees' work-life balance and about their role in corporate change. They realized that their own routine of sending e-mails late in the evening or during the weekends might drag others into work: "Sometimes I work on Saturday at 2 am. It happens like this somehow, I'm in the flow, I have creative energy. Some weeks ago I told my group that I would like only one thing: 
that they don't answer anything from Friday 5 pm until Monday morning. This was very interesting, it had the psychological effect that they couldn't stop themselves from replying. Ergo, I started to work offline." (director, man, 40) In addition, performing at above the expected level might create the idea for employees that this is the norm. Those very few men who realized their own role and impact on other peoples' working culture tried to change their own habits or communicate properly to their colleagues.

At this point, the question arises whether it is easier to maintain work-life balance as we move up the hierarchy of management, or not. It can be assumed that at higher levels of leadership work autonomy grows and one has more access to work-life balancing opportunities as well. On the other hand, as the interviews confirm, having a higher position means greater responsibility, a higher level of stress and probably more need for visibility due to the importance of the individual's role in decision-making processes. It seems that the two effects can influence work-life balance simultaneously. "The higher position we are in, the more meetings we attend and the less individual work we do. (...) It truly helps that ... when I'm asked for an hour-long meeting but I feel I can dedicate only half an hour to that topic, then I can say this in my position. But I would say that the impacts of the two contrasting effects [visibility versus autonomy] are stronger regarding the many meetings I have in relation to my position." (chief, man, 43) The top positions are perceived differently from outside as well, as seen through the eyes of lower-level managers. Many group managers believe that it is tougher to maintain a work-life balance at a higher level in the company since the workload must be greater. Other group managers, however, feel that they have less autonomy and access to flexibility than their bosses. They even perceive less opportunity for empowerment than the top-level management. In conclusion, it cannot be clearly ascertained how the level of management influences work-life balance.

\subsubsection{Female Managers, their Agency and Capabilities}

Women in the sample identified the same work-life balance policies as men, putting flexible working hours and home office at the top of their list in importance. Among women, nobody stated that the company's work-life balance support did not work, or is superficial, as some male managers did. In general they seemed to be satisfied with the company's contribution to their work-life balance. Only one issue triggered different opinions: the question of mothers' return to work and part-time options. As already mentioned, a few women strongly supported the idea of having 
more part-time opportunities, at least while children are at pre-school age. Part-time work is not available however for those in a managerial position. In addition, two mothers were against part-time work, believing that it was just a form of exploitation: "I find the 4-6 hours to be a form of obscurantism. I had a colleague who came back in part-time, it wasn't a win-win situation. The team only sees that she is never there when she's needed (...) She only gets a part of her salary. Maybe it's not the company's way, but I don't really allow the 6 and 4 hour working options. I'm quite flexible about allowing mothers to work from home. I've just sent one of them home. Also, leaving earlier we manage, but these 6 hours are not good for anybody." (head of department, woman, 44) This issue could be discussed further, but in any case it appears to be a limitation on the ability of employees who might find it a desirable option to work parttime. Based on the interviews, there are some female managers who would or would have chosen a part-time solution with all its advantages and disadvantages.

As previously described, the home office and flexible working hours were seen as tools which could help to balance work and family obligations according to female respondents as well. Women also complained, however, that they do not always have the option to use these options, primarily due to the meeting culture: "What could I change, and I even try to cross out [working in the office] every Friday to use the home office option instead. But usually this option is overwritten by corporate incidents." (head of department, woman, 42) But most managers used positive terms when talking about corporate culture and except for one group manager did not voice truly critical opinions - unlike the male respondents. They praised first of all the informal atmosphere and the human-centred way of operating. As one of the female heads of department reported, they worked not with a task- but a human-centred focus. This takes more energy and attention but that is what she believes in. Female managers even more strongly highlighted the importance of direct supervisors. Most of them feel that their supervisor is tolerant and supportive about their work-life balance needs and can count on their understanding: "I felt several times that I would rather be a waitress in my town just so I could spend more time with my family. When I reached that point, I sat down with my boss and told her this honestly. And she acted as a partner, saying, then let's change, let's hire an extra person. This is concrete support from the boss: we hired someone to help me and she is the one who tells me to use a home office. She is practically coaching me." (group manager, woman, 38) 
Most of the manager women can be described as very perfectionist, internally motivated and engaged to the company. Some find their own perfectionism bothersome and they try to reduce it. One female group manager has the feeling that her colleagues are not as ambitious as her, and that is why she has to put in extra energy to compensate for their laziness, while others simply feel that they have to meet every challenge and undertake all the tasks they face. Other managers do not find their perfectionism a problem, but they can clearly see that it is them who often generate the tasks. For example, one female interviewee believes that it would not cause a disaster if she stopped reading messages at home, but as her husband checks his Facebook all the time she does the same with company e-mails. There were only very few women who had learnt to reduce their perfectionism and say no to certain tasks: "I can say no. Some people can't, I know some. But maybe they find themselves to be somewhat irreplaceable. But I see how people are sent away. Nobody is irreplaceable." (group manager, woman, 44) Two female managers shared the perception that visibility and constant availability not only stems from personal perfectionism but is also expected from the company. They already felt that they were blamed when they used the home office. They did not talk about concrete consequences, but rather about mean comments or jokes from colleagues.

As for the question whether work-life balance is the responsibility of the individual or the corporation, female respondents also regarded it primarily as their personal responsibility, just like men. A group manager added that she tended to find excuses for her work-life balance problems such as being a mother and not having time for herself, but the reality was that she had become lazy. According to her, an individual has time for what she or he wants to have time for; consequently, she has to change her attitude. This is a good example of how corporate conditions are seen as a given. In accord, when asked about the change they could make to create better balance they referred to personal changes like planning more in advance, having a more structured schedule, reducing perfectionism, or finding their priorities: "I'm improving, for example I'm at my personal trainer from 5 to 7 in the morning instead of working [laughs] (...) My aim is to have a day when I leave my laptop in the office. I'm not there yet [laughs]." (head of department, woman, 41) Similarly to men, only the higher-level managers (in this case the heads of departments) thought about the consequences of their own behaviour. This manifested primarily in ideas about how they could help their subordinates by giving them flexible options and showing tolerance towards their 
family needs. There was only one female head of management who realized that the practises she represented might become norms for her subordinates: "Endless work can easily become the standard, and we are angry at those who leave at $5 \mathrm{pm}$, although this would be normal. (...) When being responsible for 25 people you have to represent normal standards towards them. I don't want that those things I went through in the last few years become the norm for these 25 people." (head of department, woman, 41) The female group managers however, similarly to their male colleagues, do not seem to perceive their position as being endued with the potential to make claims for work-life balance, or to challenge work norms within the company.

\subsubsection{Discussion of the Second Research Question}

Since the study of Géring (2014) it has been known that only a small minority of medium and large-size companies in Hungary find it important to represent corporate social responsibility on their websites. This company belongs to this small minority since it does use its website to advertise the importance of corporate social responsibility, including employees' work-life balance. Moreover, support for harmonizing employees' work and family responsibilities not only appears in an online representation but is included in corporate policy, primarily in the form of flexible working opportunities, help for mothers to return to work, and the extended cafeteria system. A minority of male managers were critical about the company's work-life balance policy, claiming that all the initiatives remain on the surface and do not challenge the main problems such as the inefficient culture of meetings, or the extreme workload. The majority of men, however, found company support for flexibility to represent great support. Unlike men in the research by Tóth (2007) and Primecz (et al., 2014), most of the managers saw flexibility (in the form of having the freedom to structure working hours and use a home office) as provisions that not only female employees but they could benefit from as well. Consequently, the gendered nature of flexible options was not obvious in connection with these arrangements. This statement, however, does not apply to part-time work and parental leave, the use of which could truly challenge deeply rooted assumptions about how work should be carried out. Nonetheless, the de-gendered use of flexible working hours and a home office can be seen an important innovation in the Hungarian labour market context.

Besides formal policies, support from direct supervisors plays at least as important a role in the work-life balance of employees. This finding is completely in accordance 
with international findings (Ladge et al., 2014; Rantanen et al., 2011; Kossek et al., 1999) about how informal relations within the company and workplace support (Kossek et al., 2011) can influence the use of flexible working options, but is especially valid in the Hungarian context, where managerial control is closely intertwined with informal practices and the supervisor-subordinate hierarchy (Primecz et al., 2014; Kispéter, 2012; Utasi, 2011). Most managers state that it is a relief if they can avoid the bureaucratic procedure of asking for leave. Instead, they feel that they can act autonomously in organizing their day if their absence is not strictly monitored. This autonomy increases with the level of management, and is stronger in certain departments, confirming the asymmetries of power that Primecz and her co-authors (2014) draw attention to.

Just as Tóth's (2007) work identifies, the company's pleasant infrastructure and (sometimes) corporate events were also seen as part of the work-life balance policy, although these were less determinant than in the research into Unilever, thus cannot be characterized as "work becomes home, and home becomes work" (Hochschild, 2001). Instead, one general term was used to describe family-friendliness and the support for work-life balance: Scandinavian organizational culture. The respondents stated that the informal relations described above and the democratic way of leading and decisionmaking represented the human-centred attitude of employees and leaders. Utasi (2011) argues that the Hungarian working environment lacks democratic structure in the sense that fewer regulations protect employees, the supervisor-subordinate relationship is more hierarchical and the level of distrust is higher. Many respondents feel that the Scandinavian influence on corporate culture manifests itself in the more democratic way the company functions. One potential explanation for this is that the less rigid hierarchy within the company is what makes the employees feel free to take advantage of the flexibility-related options, or that this approach generates a higher level of trust that motivates use of the home office. Those who support the use of a home office within the company find trust to be at the core of this alternative method of working: most of the supervisors believe that subordinates will not misuse this option therefore subordinates work even harder to prove their supervisors right and gain their trust. Those who led by example by using a home office themselves can be regarded as change agents, as Hobson and her co-authors (et al., 2011) call them. By using a home office and flexible working hours managers could alleviate the fear of employees of doing the same. This phenomenon confirms that it is important that managers are role-models when it comes to the development of a family-supportive organizational culture (Allard et al., 2007; 
Holter, 2007; Rantanen et al., 2011). Consequently, even if not everyone finds the institute of home office to be the best solution since they prefer to separate work and home, the majority of male managers viewed this opportunity positively. This situation is very different from the one Tóth (2007) experienced at Unilever, where working from home was considered disadvantageous.

A supportive culture has been proven to increase employees' loyalty (Rantanen et al., 2011). Most manager men in the sample claimed to be committed to the company, although the level of engagement differed. This finding is in accordance with the high (79\%) engagement index recorded at the company by the Employee Engagement Survey in 2014. Taking into consideration the long years many managers have been employed by the company also implies a high level of loyalty. Despite the high level of employee engagement, the survey showed that it is exactly the issues of work and family harmonization and the high workload that are the weak points at the managerial level.

Besides all the positive remarks about the supportive organizational culture, some managers provided concrete examples of obstacles which hindered their claims for a better work-family balance. In most cases, face time (Van Dyne et al., 2007) itself, just for the purpose of showing a presence, is not necessary, especially for high levels of management. On the other hand, it is exactly the culture of meetings that requires personal presence. Since the personal presence of managers is needed in decisionmaking processes during meetings, and there is rarely a chance to delegate such tasks to others, managers become irreplaceable, just as Halrynjo and Lyng (2013) describe. Visibility, of course, is contrary to the idea of flexibility, so even if the use of a home office and flexible working hours are supported by most of the divisions the use of such options is often overwritten by the need to be present at meetings. Therefore those managers who are critical believe that if the culture of meetings were more efficient, and more empowerment were possible, flexibility could be increased and operative tasks could be done within working hours. The Human Resources Department has also discovered this problem and is thinking about possible solutions for making meeting culture more efficient, and further supporting alternative ways of working.

The majority of managers considered work-family balance to be a private responsibility, just like Ford and Collinson (2011) found with UK managers, although the leaders of the Human Resources Department have the feeling that every employee expects them to find a solution to their problems. Most probably this observation is 
more valid for staff members. When it comes to agency, they tend to think about what personal changes can be made, how they can be more efficient, and/or how they can plan or prioritize better. Some of the actual changes managers have made to improve work-family balance are identifiable, like moving closer to the workplace to save on commuting time, or restructuring schedules to be able to take children to nursery school. The latter is important because of how leaders can provide an example of more sustainable working practices: the fact that a male director comes to work later because he wants to take his child to nursery school can mediate the message to employees that it is acceptable to prioritize fatherhood-related responsibilities over work-related ones. These scattered and occasional examples, however, may not be truly challenging the model of the ideal employee or work devotion schema since they do not exceed the level of individual strategies, similarly to in the companies observed by Primecz and her co-authors (et al., 2014). Kvande (2009) also states that it is difficult to make real change when family-friendly policies remain the choice of individuals, and work-life balance is a personal responsibility. Although the importance of these examples might seem to be overemphasized, they can still be considered important compared, for instance, to the findings of Nagy (2008) or Tóth (2007) whereby male employees did not use flexible working options at all, and did not intend to change their routines to spend more time with their families. Moreover, the individual adaptive strategies of higher level leaders may result in collective action if the role-model effect is strong enough.

The perception of who is responsible for work-family balance is different at higher levels of management: directors and chiefs were more aware of their own influence as leaders on their subordinates' work-family balance. This is crucial in situations which lack formal regulations and function through informal norms, such as with flexibility. Without clear borders, precious, family-friendly employee-driven flexibility can easily turn into exploitative employer-driven flexibility that puts even more pressure and responsibility on workers, as Hochschild (2001), Van Echtelt (et al., 200), Ford and Collinson (2011) or Brandth and Kvande (2001) found. Many enjoy flexibility and do not mind working at home or answering e-mails at night, employing the integrator boundary management style. This entails, however, the danger that the behaviour of these ambitious, engaged, highly motivated people becomes the norm, and puts pressure on other colleagues who would rather prefer to separate work and home or simply do not want to put more effort into it, into doing extra work. Confirming the 
findings of Mellner (et al, 2014) this might be problematic, especially for those who have low control over boundaries between work and home and do not feel in a position to reject these unspoken norms and expectations. This finding is in accordance with those of Lewis (et al., 2007) that, when work-life balance is taken as an individual responsibility, some individuals will be satisfied with their 'own choice' to work long and hard, while others will blame themselves for not being able to control the blurred boundaries between work and family. As some of the directors concluded, the problem with information technology is not that it makes the borders between work and home permeable, but that there are no clear rules or guidance about how to use it the right way. Therefore changes such as when directors, chiefs or the CEO decide not to contact employees on weekends, or prohibit them from doing so, might be creating some basic limits to uncontrolled and boundless work.

To sum up all this information, let us place it in the agency and capabilities framework (Sen, 2008; Hobson et al., 2011): the sense of entitlement to make claims for a better work-family balance among manager fathers was not too high, taking into consideration the fact that taking advantage of parental leave or part-time work in order to spend more time with children did not even cross the minds of such managers, similar to what Hobson and her co-authors (2011) found using a Hungarian sample. Therefore we cannot really talk about a clash between traditional organizational culture and the growing family needs of involved fathers, as for example O'Brien (2007) or Holter (2007) do, since fathers make no real challenges towards organizational culture. This can be explained primarily by conversion factors; namely, that according to gender norms men are still expected to be the main breadwinners (Takács, 2008, 2013; Pongrácz, 2001; Nagy, 2008). Consequently, even if some modern elements of fatherhood exist and fathers feel the need to spend more time (or 'quality time') with a child, the traditional separation between paid and caring roles is still strong. On the other hand, workplace organizational culture gives some room for manoeuvre in the form of the home office and flexibility, which are used by manager fathers to partly satisfy their need to devote more time to their families. Optional, non-standard flexibility and leave solutions however do not challenge the ideal employee and work devotion schema (Williams et al., 2013; Kvande, 2009; Brandth - Kvande, 2001; Lewis et al., 2007), since when deadlines are tight and projects are important, work can easily be over-prioritized. Therefore situated agency provides the highest level of variety among manager men as regards how much of a balanced life they can achieve: some 
receive more social support (Kossek et al, 2012) than others in the form of having grandparents nearby or being able to afford paid help, while others live closer to the company and have to travel less. Some managers have more supportive supervisors than others, or more trustful subordinates, or simply have better scheduling and organizing skills. The interviewees feel that they have greatest control over their situated agency, which is why they try to adjust these factors to achieve a better work-family balance, while the conversion factors and workplace organizational culture are taken for granted.

\subsection{Third Research Question: Managerial Masculinity and Involved Fatherhood}

This chapter covers the findings connected to the third research question. It focuses on how manager fathers have experienced fatherhood; the related questions include: what tasks do they consider to be primary parenting tasks, what changes has fatherhood brought to their life, and what responsibilities does their parenting involve. Additionally, manager fathers are also asked about the role of their managerial position and the importance of their work in their lives. Thereafter, fatherhood and managerial masculinity are compared in the sense of how manager men see the relationship between their parenting and leader roles, and which domain they feel more confident in. The chapter continues with a brief summary of how female managers can harmonize expectations about being both leaders and mothers. Finally, the chapter closes with a discussion about the third research question.

\subsubsection{Becoming a Father}

From the three research questions, the third was the most difficult to inquire about since identity is difficult to capture. Asking direct questions about this could have confused interviewees or resulted in banal, predictable answers. As a result, I instead asked the male managers more generally about the changes and feelings fatherhood had brought into their life, and what they found important as fathers.

Two types of answers emerged in response to the question what managers consider their most crucial task as a father: materialist and post-materialist values. Under materialist values I include the financial maintenance of the household, as well as the provision of safety and security to the family, while under post-materialist values ideas about being a role-model to children, being present and giving love are filed. These are, of course, not mutually exclusive categories; references to materialist and 
post-materialist values appeared simultaneously. As can be seen, materialist duties are closer to more traditional ideas about fatherhood since they assume that the man will be the main breadwinner of the family and is expected to provide the family with financial, existential and physical safety. Post-materialist values are more complex: moral considerations such as providing a good example, giving guidance and being a rolemodel for a child can also be regarded as characteristic of the traditional father model, making the father the head of the family. Other elements, however, such as loving the child, being available, giving care, attention and unconditional acceptance are clearly typical of a more care-oriented, involved father. One third of fathers in the sample listed these emotional duties, emphasizing that their most important task is to support their child to have a happy and fulfilling life. Being a role model, teaching values and morals were particularly popular answers, with almost half of all male interviewees finding these elements to be essential components of fatherhood. Around one third of all fathers showed a mixed picture, finding both materialist and post-materialist aspects to be important. Consequently, materialist values rarely appeared alone, but were usually accompanied by ideas about the importance of being a role-model. It is important to highlight that these comments only relate to ideas about ideal fatherhood. The length of the interviews did not allow me to ask the respondent fathers in detail about their everyday practices and routines. It is well-known that what is revealed through discourse is not equivalent to actual behaviour. Consequently, when an interviewee is hypothesized to be a more involved father, this refers only to his discourse about fatherhood.

It was common that fathers expressed a desire to spend more time with their children if possible. It seemed, however, that they were more or less satisfied with their performance as fathers, or they only had slight doubts about whether their parenting would prove successful in retrospect. Only three male managers really seemed to be suffering from not being able to represent the father ideal that they wanted to: "Now we are living as a classic family, an orthodox one. I put bread on the table and that's where my father responsibilities end. But obviously it's not good. I would like more than this. I don't know, I would like to raise happy people, and I want to take my share of that." (head of department, man, 40).

When manager fathers were asked about the changes parenthood had brought to their life, half of the fathers claimed that it was impossible to prepare for it. According to these respondents, one can read books about parenting, and ask for advice from 
relatives and friends, but reality has nothing to do with it. Even if having a baby had been a conscious decision for almost every family, the manager men experienced it as a radical change in their lives. This change took several forms and had its ups and downs. The most important shift in the fathers' lives was that they immediately felt the weight of being responsible for someone other than themselves. Some experience this responsibility as pressure, while most respondents were inclined to see the positive side of caring for someone else. This characteristic is strongly connected to the role of breadwinner; creating a secure financial background for the family takes priority, and work becomes even more important for these reasons. As one individual said, they had never worried about losing their job before they had a child. As a consequence, they now tried to avoid taking any risks and made only considered steps. Another huge change fatherhood had brought in the respondents' lives is that they had to make many compromises. This included a sacrifice of leisure time, a decline in number of meetings with friends, a lack of time for hobbies and relaxation - even reading a book or watching their favourite series on television. Time spent sleeping drastically dropped in the first years after childbirth, resulting in exhaustion, sleep deprivation and even neglect of the marital relationship due to the couples' lack of time and mutual activities. This was not easy to accept for most fathers, but as they said, fighting against these natural changes only led to conflict. The conclusion is, however, that despite every difficulty, fatherhood brings such joys in their lives that it compensates for all the sleepless nights: "On a daily level it's two shouting creatures in your well-formed life where you feel perfectly well together with only your wife. So it has really rewritten our life. But somewhere underway you incredibly fall in love with them [the children]. (...) You experience completely different joys. The Lego car my son has built, or what my daughter tells me about ponies, these are all such sources of joy one can't imagine without a child." (head of department, man, 40)

The other half of the fathers did not experience fatherhood as a radical change. This does not mean, of course, that they did not have to change their lifestyles. They also talk about compromises, exhaustion and a shift in priorities but they felt prepared for all of this, and tended to highlight the positive elements of fatherhood. "With my wife we don't take things seriously. Not even this. This doesn't mean irresponsibility, of course it was a big change when a child entered our life, and now we have to wake up at 3 am to prepare food for her, or change her diaper, but we see absolutely the positive side of this." (group manager, man, 35) They add that they did not see the change in 
their lives as radical because it did not happen from one day to the next. They did not realize that they had had to give something up, but just noticed that the old schemes in their lives were no longer valid. Consequently, fatherhood brought about a change in their lives, but not a sudden, radical one, but rather a slow and predictable shift in daily routines and priorities.

It does not appear either that a father's identity arrives immediately with the birth of a child. Not all the interviews covered this perspective, but around a quarter of all fathers talked about slow evolution of the bond between them and their children. They report that they did not know how to start making contact with their new-borns. In their eyes, the baby seemed to be someone distant, not completely human yet, too fragile to be touched. "What I remember is when my wife was brought to the operating room, she had a caesarean operation, I was very worried about her. I didn't have any child yet. And when they [the twins] were out of my wife's womb, they still weren't my children yet. So there were those two humanlike things which they wanted to put into my arms, but I said no, find a specialist who won't drop them. For months they were only shitting sausages, two pieces of meat with whom I didn't know what to do." (head of department, man, 40) Two men even mentioned feeling discomfort and irritation around their children. They did not see their role in parenting at the beginning, except for a feeling of responsibility, that was very strong. "Why aren't we told in school that it [parenting] sucks that much? (...) I don't think that my personality has changed, but most probably I'm still going through this circle of change, sometimes disappointment, denial, about losing certain parts of my free time. My children are very cute, very intelligent, very clever, really, but they take lots of energy and time from my wife and from me too." (head of department, man, 39) A few male but even some female interviewees explained this by referring to gender. They claimed that mothers make a connection from the start while men do not need to play a big role at the beginning of a child's life. But then an emotional attachment develops slowly and seamlessly. As these men say, they adapted to the new situation over time, and the bond formed as the children grew and started to be able to express themselves: "There is a distance. Most probably not with every father, but for me it took 3-4 months to first feel that this child was mine and that we have a relationship. And I had to wait 3 whole years to also have that sparkle I had seen in the eyes of a mother and child looking at each other." (director, man, 40) Only very few fathers described the process differently, claiming that they felt emotionally attached to their child from the very beginning, since the time 
they knew their wife was pregnant. For these individuals the feeling came naturally and therefore they did not understand those men who had difficulties with their new-borns.

The limits on the length of the interviews did not allow me to ask fathers about concrete tasks they undertake in parenting, but, fortunately, many interviewees made references to these. This probably resulted in the collection of more valid information, since interviewees did not feel the pressure to answer in accordance with social expectations. From the examples mentioned, it can be seen that fathers primarily undertake tasks which may be classified as interaction and availability according to the typology of Lamb (et al., 1987). Most fathers are engaged in the evening routine during the weekdays which includes helping with dinner, bathing and putting children to bed. Reading bedtime tales is an important part of the evening routine for many families. As if the interviewees felt a need to explain the lack of time spent with families during the weekdays, they often emphasized the importance of the weekends as compensation. The term 'quality time' was used a few times at such moments in the context that children do not need to be played with much, but this requires complete concentration. The weekends are the time when fathers can pay undivided attention to their children and, for example, play with Lego, watch cartoons, or tell tales in the case of younger children. With the older children it is possible to do sports, play billiards, go hiking and cycling or even read Harry Potter books together. For school-age children fatherhood activities also cover learning together and checking homework. Sometimes these activities occur in the frame of father-son or father-daughter programs, and it turned out in a few cases that wives were doing the housework in the meantime.

Not much was said about chores but the interviews gave the impression that manager fathers did not play a big role in doing household duties. As was already explained, most interviewees either hire a cleaner or leave a partner to do chores, especially when she is on maternity leave. The tasks they mentioned that they did were rather administrative in nature, such as paying bills and going to the council, or undertaking more gender neutral housework, such as shopping or cooking. A few men really seemed to be engaged in cooking, even baking bread for the weekend breakfast, but they stated that they enjoyed this activity. In general, some interviewees admitted that their wife managed household tasks and played the greater part in parenting, especially in the first years. In these first periods most of the fathers stay alone with their children only for a few hours while their wife goes to the gym or asks for some rest. Later, however, when the child is older, the bond strengthens as time is spent alone 
with a child more frequently. One of the directors admitted that in the first years of his children's life he was not available at all since he was doing a job which required him to commute, and he spent only weekends at home. Since he could not maintain this way of life he quit his work and stayed at home for 10 months before finding his next job. He described these 10 months as compensation, or a correction for the first missed years. He claimed that he had given complete attention to his children, and spent all the time with them. It is important to know, however, that during this time his wife was still at home on maternity leave so it is not clear what active participation exactly occurred.

The responsibility tasks from Lamb's typology (1987) include taking a child to kindergarten or school and bringing him or her home from extra classes, or sports training. Many fathers regularly take their children to school on the way to work in the morning. Usually, the duties are shared and in these cases the wife picks the children up in the afternoon since the fathers are still working. Sometimes they do the reverse and the fathers are responsible for the afternoon duties, especially when the children have extra classes and finish late, which also suits better the father's working schedule. "Today, for example, my youngest son insisted that I bring him to nursery, because it's been a long time since I could. So I took him. And it felt very good." (director, man, 42) The transport of children seems to be a challenging logistical task for many families which requires constant communication between parents. It often happens that a lastminute meeting ruins a planned schedule and the other parent has to deal with the unexpected situation. According to the interviews, there are precedents when manager fathers use flexible working hours to manage these cases.

\subsubsection{The Role of Work}

It is not easy to measure what work means to individuals. For some people work may only be a financial tool for supporting their existence, while for others it might be part of their self-realization. Based on the entire set of interviews and the answers given to work-related questions I tried to locate the place of work and careers in the lives of male interviewees. It would be an exaggeration to narrow the picture to the two extremes of working only for a salary, or having work as the core of identity, especially since almost all the employees I interviewed expressed a certain feeling of engagement to the company and claimed to like working there.

There are some manager men who obviously consider their work to be more than just a job. These are the managers who usually do not mind answering e-mails at 
night, who want to be up-to-date about every project, who are perfectionists and are proud of their results and of the company as well. For some, their work is a hobby as well. As one of the directors put it, some people need to do extreme sports for adrenalin; for him, the purpose is served by work. Most probably this is the perspective of the interviewees that best described what managerial masculinity is.

Those who see their work as less determinant highlight rather the importance of family, adding that they would not want to sacrifice their life for work. "This is only a workplace. In fact, it's not worth falling ill or sacrificing your marriage for. It's difficult to recognize this, especially when you are in financial straits (...) Placing my work before my health and family is short-term thinking." (group manager, man, 36) These individuals prefer to separate their work and family life and can stop themselves from constantly checking their e-mails. As already explained, this does not mean that they do not like their jobs or do not try to do their best. But they do not chase work success at all costs, or do so no longer since they have had children.

Manager men were also asked what parts of their work they enjoy the most. The answers given to this question present a varied picture: in first place the creativity and variety of the work was mentioned. Managers like innovative, interesting tasks and the fact that there is always something new to learn which prevents them from getting bored by routine duties. The often challenging projects make them think and focus hard, creating a busy and exciting pace for the day that many enjoy. Their managerial jobs are varied and include interactions with clients, subordinates, having meetings, making decisions, analysing, and so on, which allows them to participate in different kinds of tasks.

The second most frequently mentioned element was the influence and responsibility they have in their jobs. They enjoy the autonomy and freedom they have in decision making. They do not consider the responsibility their work entails to be pressure but rather a form of power which allows them to influence outcomes. They believe that they are creating something useful and valuable and have the ability to affect important processes, to change situations. Some even add that they enjoy the success of witnessing the results of their own work: how they can build up something from scratch and also the positive feedback they receive from clients or from their supervisors and colleagues after a successful project. "I like feeling that my work has sense. When I bring in an initiative which I really believe in, and it becomes something, has an influence on the company. (...) I was never attracted by the power which comes 
with the higher position, but I'm interested in doing more complex tasks and the responsibility, and the fact that I have influence. This ambition is needed for leadership." (head of department, man, 39)

What managers like the most in their work in third place is the team and teamwork. These managers enjoy their everyday interactions with their colleagues. They consider having a good atmosphere, being able to work well together and create results as a team to be the most pleasant characteristic of their job. This perspective is very similar to the next point interviewees talked about, which is leadership. Similarly to the previous, this also has a focus on human interactions but is rather connected to coordination than cooperation. Some explain in detail how they enjoy seeing their team members progress and develop with their help. As one of the group managers said, this involved not only leading but coaching too. A good leader has to find ways to motivate people and help them to bring out the most of themselves. Consequently, the leader picture the interviewees described is far from autocratic in style and is close to the cooperative alternative of leadership. This includes power and influence in the same way, but builds it on trust and cooperation.

Finally, some male managers mentioned the nature of the work in the first place. For them, the sector in itself is the most important element since they always had an interest in human resources or engineering. They could not imagine themselves working in a different area even if they were offered a better position. Their main motivation is the subject itself, and the goal of becoming an expert of the chosen area.

\subsubsection{The Relationship between Management and Fatherhood}

The most important part of the third research question relates to the relationship between and the reconciliation of managerial and father roles. In order to map this aspect, the interviewees were asked about how managerial and parental roles were compatible with each other, what were the similarities and differences between the two roles, and whether they faced any contradictory expectations or difficulty with switching between them. As can be seen, the question focuses rather on potential conflicts, although interviewees did not seem to be diverted in this direction. Nonetheless, in order to avoid forcing the idea of conflict onto respondents, they were also asked a less direct question about the relationship between managerial and parental roles, focusing on which field of life the interviewees feel that they were most successful or confident in. I found the question to be open enough to cover not only the 
work and home domains, but other spheres as well, and to explore which domain managers find more complex or more valuable.

According to the answers given to the question about the relation between parental and managerial roles, individuals have two types of approaches. The majority, about two thirds of all interviewees, identify a relationship between the two roles and find them very similar to each other. The other third of the respondents, however, consider the two roles to be independent and have nothing to do with each other.

Those who think the roles are similar believe that being a parent and a manager includes plenty of parallel expectations such as being a role model, showing a good example, and being able to encourage and motivate others. As some state, one has to be a good leader of a team, as well as a family. They define leading as taking responsibility for others and providing guidance, motivation and support. Thus one does not necessarily need to be cold or strict with a child just because he is a leader, but consistency is indispensable. Subordinates need teaching and help, just as children do, only in different things and at a different level. Emotions and human factors play a great significant role, not only in family life but also in work: "Emotions have a place at work just as much. A leader can become a good leader only if he is able to notice every flutter of his colleagues and can react with empathy and attention. Only in this way do relationships become good and stable. So there is no such distinction that work is a place for rationality, while private life and home are about emotions." (group manager, man, 37) Some men even described themselves as the fathers of their team, emphasizing the coaching side of leadership and referring to their subordinates as their children at work. However, they drew attention to an interesting phenomenon: that subordinates tend to lose their independence and initiative and act like children when someone is standing above them. A director stressed that, as a leader, he has to treat his subordinates as equals, not directing them, only delegating tasks, otherwise employees very easily accept the hierarchy and become the children in the relationship.

In addition, those interviewees who see a connection between the roles also emphasize that one cannot behave completely differently in one role than in the other. This does not mean that they behave in totally the same way in the workplace with their colleagues as they do at home with their partner and children, but on the whole they see themselves as complete, integrated individuals with a certain personalities and habits. Therefore, if they are generally cheerful, extrovert and easy to make friends with, then they are not capable of acting strictly and autocratically either as parents or managers 
since this would be contrary to their character. Consequently, these interviewees feel that they represent very similar values and attitude as managers as they do as parents. Accordingly, they have no problem with changing from one role to the other: "Thanks to managerial self-knowledge training I just had the opportunity to experience how others see me from outside, and the two roles were amazingly overlapping. I had to face the fact that I behaved the same way at home as here. (...) When here they described me as a good old grandpa, then I thought yes, this is a projection of my home role." (group manager, man, 39)

Within the group of interviewees who assume the existence of interaction between the managerial and parental roles, many went beyond talking simply about similarities and highlighted the positive influence of one roles on the other. According to this perspective, the two roles are not simply parallel in many regards, but some of the skills learnt in one domain can be used in the other. This is basically the definition of enhancement or positive spillover. These interviewees mentioned perfect examples of this in both directions. By being fathers the male managers had to become more patient, attentive, empathetic, and to use more care and emotion in their communication which they later found useful in their role as team leader as well. Similarly, many of them started to apply management techniques in terms of their motivation and communication at home to achieve consistency and order. "I often say that you have to talk with a CXO the same way as you do with a child. Sometimes you learn from a better storybook about how to make a presentation. I think they [the two roles] rather support each other, since they brings rationality to the private life, and empathy to the world of work." (group manager, man, 35).

However, not everyone believes that there should be a relationship between work and family roles. Around one third of the interviewees do not see any similarities between being a manager and a parent, but rather want to treat those roles separately. They do not believe that they should behave with their children the same way as they do with their colleagues, or to bring too much emotion into the field of engineering, for instance. A male head of department explains that this not only concerns the differences between managerial and father roles, but one simply does not behave the same way with a boss as with one's friends or partners. Therefore it rather involves making a distinction between private life and work than separating father and managerial roles.

The other question about the relationship between roles asked in which life domain interviewees feel most successful. The general answer indicated a holistic view. 
One third of managers believe that they perform well in every sphere of their life. They were able to find something to be proud of in every domain, such as receiving positive feedback at work, or living in a harmonious partnership and having healthy and beautiful children. They add that this does not mean they could not be better as managers or improve as fathers, but they believe that one domain cannot be improved without the other spheres deteriorating. Consequently, they find their lives successful in the way that they manage to maintain a balance in terms of satisfying every life domain: "I think you feel successful only as a whole, otherwise you don't. Because those who state that they are successful at work and sacrifice everything for this do it for long-long years and find their success in it, but neglect other things. But I think they will feel emptiness when they look back in $x$ years." (head of department, man, 33)

This parameter might be difficult to compare since the meaning of success may be different at work and home, and can even change in time regarding what is perceived as truly important. Some fathers explained that evaluating their parenting is a more complex and abstract task than evaluating their work performance, while others thought the opposite, and talked about the instant feedback they received from little children. "I find work easier from this point, because I arrive, I do my job, they are satisfied with me and that's it. (...) I'm satisfied with the little responsibility I have. It's more difficult to evaluate the things at home, to tell when you are successful. I dunno, someday when my son graduates from university and has his first job and we sit down to talk with a beer in our hands, maybe I will get more feedback." (group manager, man, 43) Although the question was not narrowed down only to work and family, the interviewees rarely mentioned other fields like being successful in their hobbies or popular among their friends. This can be explained by the frame of the whole interview which rather focused on work and family, but also by the fact that people find work and family domains to be more important than others.

Some more interviewees represented the same holistic approach, but used a more critical view about their own performance. They would not call themselves successful, since they do not believe that someone can be completely successful in every regard, but neither do they feel that they lack anything serious in their lives. They say that their lives are going well, they are managing to perform to the expected level in every field, but they talk less proudly that the previously mentioned managers. They even say that they often experienced downturns and have bad days, but generally they feel that their lives are balanced. Two male managers added that they needed to improve 
as husbands, since besides the paternal and managerial role, they had neglected their duties as partners.

It happened less frequently that interviewees named one certain area where they felt the most confident or successful. Only very few managers believe they perform the best at home, partly because they find this domain to be the most important and they feel proud of their children, or the fact that their marriage is still working well while they see more and more divorces in their environment. On the other hand, two male managers chose home as their most successful domain because they believed they could achieve more at work. Similar are the reasons among those few managers who named work as the domain where they felt the most confident. One male interviewee considers himself more successful in his job because he has doubts regarding his own performance as a father. He finds he has more confidence at work where the rules are simpler and the tasks clearer than with parenting.

All in all, this question did not lead to clear distinction between those who feel more successful as parents and those who perform better as managers. The answers show that when it comes to the general evaluation of their lives, the interviewees like to view it on a global scale with all its ups and downs. It is not obvious though, why people feel more confident in one domain than another: for some it is because they find (for example) family to be the most important thing in their life, while for others this domain compensates for insecurities experienced in the domain of work.

\subsubsection{Female Managers and Motherhood}

Female interviewees were not asked about the changes motherhood had brought to their lives since the focus of the research was on fatherhood. They were asked, however, about the role of work in their lives, as well as about the harmonization of managerial and mothering roles. It was highly visible that for many manager women work was an important part of her identity, and they emphasized that despite all the difficulties they could not give up their careers to be 'only' mothers and housewives. Only one female group manager in the sample simply claimed that work was only about earning a salary: "I'm not career-minded and I have never been. That's why I never wanted to take a leader position because for me this is not of primary importance now. Work is work. I make the most of it, but when I leave the office and close the door behind I leave everything here and go home." (group manager, woman, 39) Amongst what female managers like the most about their work power-related concepts are 
awarded somewhat less weight than by male interviewees, although these elements also appeared: two women mentioned influence and responsibility, while two other female group managers shared the opinion that being a leader was the best part of their work: "I like that I'm leading a team from which I know each member very well. Our relationship is more than boss and subordinate. I like that they trust me so much, and ask for my opinion, and that I can direct this little team in a way that we create good results together" (group manager, woman, 40) Even from this quote, however, it can be seen that the human factor and cooperation are important elements of leadership. Thus, not surprisingly, the importance of teams and colleagues are also determinant for female managers in the enjoyment of their work. The most important feature, however, is the creativity and variety of the work that always brings new challenges.

When answering the question about the similarities and differences between managerial and mother roles, with the exception of one group manager everyone saw similarities between the two roles. Some see it from a holistic perspective, avoiding separating the different roles in their life. Others find that consistency and a certain level of discipline is just as important in motherhood as at work, or the reverse, that just because they are leaders, they are still emotional. They often characterize themselves as the mother of their team or division. As described in male interviews, this might result in subordinates tending to act as children. According to one of the female group managers, her colleagues were working very autonomously until they became a team and received her as their leader. Since that point they had started to approach her about every little question, as if they could not solve those problems alone. More female managers said that at home as mothers they were more emotional, and sometimes even inconsistent, while at work it was easier to be straightforward since it is a different environment. To the question in which life domain they feel more successful or confident, most women, just like the male respondents, held a holistic view. Those who held other opinions either found that they should aim even higher in terms of their careers, or shared their doubts regarding their parenting, concluding that they felt more confident at work. Three female interviewees mentioned briefly during the interview that they had used the help of psychologists after their divorce, or taken their child to a school psychologist. They all talked about it slightly embarrassed, as if admitting something shameful, although their final conclusion was that they were satisfied with their lives. 


\subsubsection{Discussion of the Third Research Question}

The reasoning behind the third research question was built around behaviourbased conflict connected to the dichotomy of managerial masculinity and involved fatherhood. It was known that most of the Hungarian fathers are not involved fathers in the sense used in Nordic societies, since the taking of parental leave by fathers is very low. As expected, in the sample of manager fathers no one had taken parental leave in addition to the 5-day paternity leave. Some contradictions could have been still assumed between the demands of being an ideal employee and some elements of a more caring and nurturing approach to fatherhood. This assumption was also based on some Hungarian empirical findings (Spéder, 2011; Pongrácz - Molnár, 2011) which concluded that Hungarian fathers are expected both to be the traditional breadwinners of the family as well as to devote more time and emotional care to children. Consequently, even if the level of involvement in child rearing is far from the Nordic level, together with the traditional expectations of providing economic and financial security for the family it can still lead to dual pressures on fathers.

The interviews confirm these presumptions: behaviour-based work-family conflict can be a source of problems in fathers' lives, although its frequency and severity is far from similar to the level of time-based and strain-based conflict that was experienced. Behaviour-based conflict appears in two forms: in the form of dual expectations and in difficulties switching between managerial and father roles. The latter concerns only a very few men in the sample who complained that they brought home the commanding style they used at work. It was not necessarily children but rather wives who were bothered when their husband treated them as a subordinate at home, and could not leave their managerial styles behind. More manager fathers in the sample, however, found the other type of behaviour-based conflict that derives from contradictory expectations to be problematic. Just as Spéder (2011) and Pongrácz and Molnár (2011) describe in their research, expectations towards fathers are neither clearly traditional nor clearly modern: they are responsible for both providing financial security for the family and taking care of children. They found emotions, availability and showing a good example to the child to be as important as financial security. These are not only external expectations and demands, but the discrepancy appears at the level of their own contradictory desires as well. The gap between the fathers' desires is especially significant when they feel highly engaged to their work but also long to be 
present in their children's lives at the same time. Nonetheless, as Spéder (2011) found, fatherhood is considered to be indispensable for a fulfilled life by the majority of Hungarians, and men in the sample also agreed that fatherhood was the best thing that could had happened to them.

The contradiction between traditional and modern expectations, however, is even more visible in the context of economic crises and financial insecurities: a fathers' role as breadwinner is even more strongly highlighted in insecure situations that can overwrite the importance of caring duties. This means that, despite the mixed elements of fatherhood, when fathers are forced to make a choice between breadwinning and caring, providing financial security is still more expected from them. This observation accords with the results of Szalma and Takács (2013) that when men feel that their jobs are insecure, they may be more accepting of traditional attitudes. Thus, a sense of risk and economic insecurity affect agency and the capability to make a claim for a better work-life balance (Hobson el a., 2011) and leads back to traditional division of labour. This also confirms the finding (Williams et al., 2013; Ladge et al., 2014) that fathers are more trustworthy employees than men without children, since due to their responsibilities as breadwinners they will not risk losing their jobs.

It is not insignificant, however, that despite the low level of freedom they have to claim a better work-family balance, few manager fathers undertook any concrete activity to change their situations: quitting a former company that prevented one employee from spending enough time with the family; cutting back on work after becoming a father, or rescheduling days to be able to take children to school or bring them home. Some men sacrifice the time they could spend with their wives in exchange for spending more time with children, which resulted in feelings of guilt. Still, many of the fathers expressed their desire to spend more quality time with their children, like Takács (2013) found in her research, but similar to other studies (Rehel, 2013; Hobson et al., 2011) they legitimized their absence by referring primarily to economic rationality. Biological necessity, like breastfeeding or the relationship between the mother and child, was rarely mentioned and only in the context of how the feeling of fatherhood developed. Although Plantin (2007) found that Swedish working-class fathers saw fatherhood as a natural part of a planned life course, while middle-class fathers reported revolutionary change, manager fathers in the Hungarian sample mentioned both phenomena. Although almost all the children were planned, for some fathers the bond with their children only formed after a few months, and they 
experienced radical changes in their lives after becoming parents. Others had felt immediately close to their child and perceived fatherhood as natural, just like the working-class men in Plantin's (2007) research. Regarding their parental involvement (Lamb et al., 1987), most activities can be categorized as interaction or availability. Involvement in responsibility appeared in the form of taking a child to school or home. Besides taking care of children, manager fathers in the sample did not seem to be actively participating in chores. This is in accordance with the finding (Johansson Klinth, 2008; Rehel, 2013) that being a child-oriented man does not necessarily mean gender parity. In addition, even men's child orientation may more strongly involve 'picking out the good bits', just like in the case of the highly-visible-interaction type of involvement (Lamb et al., 1987; Rehel, 2013). It is important to add, though, that due to the interview method, only the discourse could be captured, thus the analysis of actual behaviour and participation in tasks is based on interviewee self-reporting.

Related to the role of work in manager men's lives, it may be assumed that power and control are key elements of a managerial position (Collinson - Hearn, 1996) especially through its economic, social and symbolic values (Bowman, 2007; Burchielli et al., 2008; Williams et al., 2013). The mechanism of greedy organizations works here in the sense that a company can build close links with the social identity of managers, making knowledge work a major source of elite status (Coser, 1974; Burchielli et al., 2008; Lewis et al., 2007; Kvande, 2009). Or, as work devotion schema (Erin - BlairLoy, 2014) claims, there is an implicit contract between the employee and the company which assures the employee that his sacrifices of time and energy will be honoured. As some managers explained, they enjoyed the feedback they received after a successful project and this appreciation made them satisfied and motivated to be even better next time. Such a feeling of commitment and engagement to work can both justify and fuel very long working hours (Williams et al., 2013).

This also relates to responsibility, just as in the case of fatherhood: interviewees often mentioned that they felt responsible for their team, as if the subordinates needed attention and guidance just like their children. Some men even saw themselves as the fathers of their team. Therefore engagement might be rather understood in terms of informal relations between colleagues, rather than commitment to the company as an abstract entity. This finding might be in accordance with Connell's (2006a) and Acker's (2006) observation that the distance between managerial and non-managerial staff is short, and management has been brought socially closer to employees. In some ways 
this is similar to the findings of Hearn (1992) that drew attention to the mixed elements of patriarchy and fratriarchy, where men express solidarity and cooperation with each other yet relate to each other through processes of patriarchal and hierarchical authority. This duality emerged in the interviews: many managers described team work as the best part of their position, while others highlighted the leadership. Competition among employees and the fear of lagging behind (Halrynjo - Lyng 2013) appeared in those highly motivated and engaged managers who always want to be in control, do not like delegating tasks and hate to be left out from decisions. This was especially contradictory in the cases when these men who were chasing this kind of visibility and control over every process otherwise lacked control in boundary management since they were not able to reject a task or draw limits. As Collinson and Hearn (1994) say, new technology and equal opportunity initiatives might threaten masculine identities defined by control and power. This phenomenon does not only concern men, however, but some women in the sample as well. Consequently, power and control-related features attract many men in managerial positions, as assumed, but in the meantime cooperative elements are just as important as components of managerial masculinity. Similarly, Kerfoot and Knights (1996) claim that managers are required to reveal themselves as human beings, even if this indicates weakness and vulnerability. Female managers emphasized their identification with work similarly to men, consequently, there were no significant gender differences in the construction of managerial identity. As Collinson and Hearn (1994) highlight, managerial masculinities take several forms. What seemed sure from the interviews is that most of the managers avoid using an authoritarian style of management since requiring unquestioned obedience and rejecting debate would run contrary to the Scandinavian understanding of democratic corporate culture.

When talking about the relationship between the managerial and parental roles, manager fathers tended not to concentrate on the role of conflict and potential contradictions but rather represented a holistic view: they see themselves as integrated individuals who cannot be categorised only as managers or fathers. When comparing the two roles, the manager fathers found many parallels. These concern not only similarities but skills and knowledge learnt in one domain that can be useful in the other, that are perfect examples of positive interactions: namely, positive spillover and enrichment. Consequently, managerial and father roles appear to be strengthening each other, rather than causing conflict. 


\section{CONCLUSION}

The purpose of this thesis is to contribute to the Hungarian literature on the work-family interface by analysing manager fathers and the work-family conflicts they perceive. The dissertation was structured along the three types of work-family conflict described by Greenhaus and Beutell (1985) namely, time-based, behaviour-based and strain-based conflict, also including the organizational and gender context. Three research questions were formulated: how do manager fathers experience conflicts between their work and family lives; how does the organization they are working for influence their capability of achieving work-family balance, especially in the frame of flexibility; and finally, how do discourses on managerial masculinity or involved fathering affect their work-family balance. Although the focus of this thesis is the relationship between work and family life, in the interviews the broader and generally more well-known term 'work-life balance' was used to avoid excluding potentially important information not connected to work and family domains.

From a gender viewpoint, the research draws attention first of all to the importance of including men in work-family analyses. Moreover, the findings justify the 'within-gender' concept (Cinamon - Rich, 2002; Martinengo, 2007) and support the proposition that there are significant differences between men and in terms of how they perceive their work and family harmonization. Although the sample was very homogenous according to demographic characteristics, men evaluated and defined their work-life balance in different ways, had various strategies and preferences regarding flexibility and boundary management and distinct notions and experiences about fatherhood. Interestingly, there was less difference between men and women in the sample than within genders, confirming Hochschild's (2001) finding that men and women have similar experiences when it comes to harmonizing work and family. Both men and women primarily perceive that conflict runs in the work-to-family direction; while the demands of the home domain can be more easily excluded from work. In the case of men, this was expected based on previous empirical results (Burchell et al., 2007); women, however, are usually found to experience more family-to-work conflict (Greenhaus - Parasuraman, 1999; Hill et al., 2004). The contradiction can be explained by the fact that female managers have adapted to male working norms. In addition workplace can serve as 'haven' (Hochschild, 2001) when rules, responsibilities and 
feedbacks are clear, while domestic life is often unmanageable and unstructured compared.

Although several previous studies (Van der Lippe et al., 2006; Ladge et al., 2014) found that men in post-socialist countries face less work-family conflict than Nordic or Western societies due to conservative gender attitudes and the traditional division of paid and unpaid labour, the consequences of work-family conflict concern Hungarian men as well. The results are in line with former studies (Ladge et al., 2014; Allard et al., 2007) stating that primarily those men are concerned with work-family conflict who live in dual-earner households. Consequently, a more egalitarian division of labour implies similar challenges for men and women in terms of the harmonization of work and family life, although men's contribution to household chores still lags behind women's share, who are burdened with the 'second shift' (Hochschild Machung, 1989). Even a traditional division of labour does not guarantee a lack of conflict situations in men's lives, even if corporate wives (Kanter, 1977) provide a significant amount of both emotional and instrumental social support for their husband's careers (Kossek et al., 2012; Aycan - Eskin, 2005; Neff - Karney, 2005) often by sacrificing their own career progress.

Moreover, choosing managers as a sample also proved to be relevant: the interviews, together with the Employee Engagement Survey, confirm the findings (Allard et al, 2007; Mayo et al., 2011; Szalma, 2014; Geszler, 2014) that managers are even more exposed to the danger of work-family conflict than other occupational groups. Although they have more access to organizational family-friendly and work-life balance programs, and have more work-related autonomy (Van Echtelt et al., 2009; Hobson - Fahlén, 2009), it is exactly this freedom and flexibility that puts the responsibility for work and family balance on their shoulders. Therefore, not only longer working hours and greater emotional and mental pressure, but primarily the greater level of responsibility characterizes their jobs in a post-Fordist working environment.

In the conservative Hungarian context it is remarkable that men in the sample recognised the importance of work-life balance in their own lives and did not treat it as an issue relevant only to women. The majority of men perceived that there existed certain problems deriving from work and family demands. Although most of them had mixed feelings and reported about temporary or less serious problems they could cope with, one group of men expressed complete dissatisfaction regarding their current work- 
life balance. Manager fathers in this sample experienced time-based conflict most frequently and considered time squeeze the most serious problem in work and family harmonization. Lack of time for family is not only crucial from the children's viewpoint, but can seriously affect the quality of partnership, although less research is focusing on that aspect. Strain-based conflict was also a frequently identified phenomenon, although the severity differed among interviewees. Time-based and strain-based conflicts are connected in the sense that a feeling of general busyness and a time squeeze, typical of the post-Fordist time regime (Lewis et al., 2007; Kvande, 2009), as well as a lack of time for relaxing activities are partly the causes of stress. On the other hand, having a managerial position involves a higher level of job stress, as certain studies indicate (Mayo et al., 2011; Szalma, 2014), especially at the top level of management. As a consequence of job stress, it not rare that men in the sample feel impatient and frustrated, often projecting their negative feelings onto family members.

The least frequent type of conflict identified was behaviour-based conflict, although I find this component the most original element of the research in the sense that previous Hungarian studies mainly have a time focus (Nagy, 2008; Tóth, 2007; Primecz et al., 2014; Sebők, 2015), or occasionally, a focus on strain (Utasi, 2011; Blaskó, 2006) and even international research often neglects investigation of behaviourbased conflict (Rantanen, 2008). Behaviour-based conflict is also connected to the other two conflict types, since it is primarily due to manager fathers' feelings of a lack of time of that they cannot fulfil both their roles as provider and involved father which results in tension and a sense of guilt. On the one hand they have to prove themselves to be committed employees, as in the ideal employee concept (Acker, 2006), but also present and available for their children in terms of emotions, care and attention, like an involved father (Wall - Arnold, 2007; Johansson - Klinth, 2008). Hungarian fathers in general are found (Takács, 2008; Pongrácz, 2001; Harcsa, 2014) to be far from the ideal of the involved father experienced in Nordic countries (Hobson et al., 2011). On the other hand, there has been a slow increase in the time fathers spend with children (Pongrácz Molnár, 2011; Harcsa, 2014) and an understanding of the need for quality time (Takács, 2013) which together with expectations about the need to fulfil the role of breadwinner role might create dual pressure on Hungarian fathers, just as Spéder (2011) describes. As Spéder (2011) and Pongárcz and Molnár (2011) conclude, expectations towards Hungarian men cannot be simply labelled either traditional or modern since are mixed. Similarly to the findings of Takács (2015) some fathers 'slowed down' after reaching a 
certain satisfying point in their career and becoming a father and learnt how to prioritise being part of a child's life more than chasing after work success at all costs, although this development was not always easy. Those fathers perceived the highest level of conflict, who realized their absence as fathers but could not give up on their career dreams at the same time.

Fatherhood implies responsibility in terms of both material aspects, like providing financial security and safety, and post-modern elements, like showing good examples and transmitting values. Emotional and caring tasks, however, like being available for a child, devoting time and attention, but above all, love, were even more frequently mentioned than material elements. On the other hand, through their increased responsibility fathers become even more 'trusted workers' (Williams et al., 2013), since they do not dare to risk their jobs as key elements of their role as providers. This fact is even more valid during periods of crisis when insecurity and economic factors motivate couples to accept traditional attitudes and the division of labour (Szalma - Takács, 2013) and 'pragmatic realism' (Ladge et al., 2014) which can dominate the desire to be caring and nurturing fathers. Thus economic reasons can still legitimize the absence of the father from family domain, which explains why behaviour-based conflict is the least frequent among the conflict types. Findings indicate that having a more child-oriented attitude, however, does not involve more active participation in chores, similarly to the results of previous international research (Johansson - Klinth, 2008; Rehel, 2013). Household tasks and the less visible, routine components of child care are still the wife's responsibilities, although some men in the sample admit that this is not a genderequal way of dividing up tasks.

The interviewees' sense of responsibility for their families is strong, as it is also for the colleagues and teams they lead, since they are often seen as 'greedy children' as well. Both manager men and women feel committed to the company and their work, and thereby greedy organizations can create links with the social identity of managers (Coser, 1974; Burchielli et al., 2008). The respondents' managerial masculinity is constructed by power-related elements (Collinson - Hearn, 1996) like control and competition, but also cooperation, fratriarchy (Hearn, 1992) and solidarity between colleagues. The disadvantages of the centrality of control in a managerial position appeared with those men who felt trapped regarding their boundary management styles. It is they who are afraid of lagging behind and missing something, and they who always try to make themselves available to monitor every process, even during holidays, and to 
be irreplaceable at work (Halrynjo - Lyng, 2013). Consequently, they do not feel that they are in a position to refuse a task, or to draw the lines between work and home, even if they claim to. Thus they are chasing control in their work which they lack in terms of the balance and boundaries between work and family. When talking, however, about the relationship between managerial and father roles most of the men highlighted the similarities between the two domains; moreover, many of them raised examples of work-family enrichment and positive spillover. Accordingly, fatherhood can bring emotions, care and attention into leadership; a finding that confirms that involved fathering is beneficial to organizations (Ladge et al., 2014; Allard et al., 2007), while managing and organizing skills are also useful in parenting, although in the literature less is said about the positive effects of work on family life. Managers in the sample do not significantly identify either with managerial masculinity or with involved fatherhood: their style of managing involves more cooperation and emotion, while their fathering is less involved than in it is Nordic or Western societies.

Different individual adaptive strategies are preferred for managing work-family contradictions: based on the concept of boundary management style (Kossek and Lautsch, 2012) some choose to integrate home and work, while others prefer to separate the two domains. Both can work well when managers feel in control over their preferred boundary management style and do not perceive restrictions from the company. In accordance with Mellner's (et al., 2014) statement, having control over a boundary management strategy is more important for reducing work-family conflict than whether an individual prefers to segment or integrate the domains. Those integrators who do not perceive that they have control over borders feel frustration and work-family conflict. This lack of control can be explained by poor prioritization and organizing skills, but first of all, by the fear of lagging behind (Halrynjo - Lyng, 2013) and being excluded from decisions. This shows well the danger in blurred borders and the ostensible freedom derived from flexibility: the lack of clear limits puts great pressure and responsibility on employees, who become the driving force of endless work and blame themselves for not having control over the boundaries between work and home, while the role of the corporation remains invisible.

The company's representation of family-friendly attitudes in its online communication means that it belongs to the minority of medium and large-sized firms which put emphasis on work-life balance on their website (Géring, 2014). By work-life balance workplace support, almost all managers in the sample regardless of gender 
referred to flexible working hours and use of a home office. These employee-driven flexible arrangements do not only target women, like at many other companies (Tóth, 2007; Primecz et al., 2014) but men benefit from them as well. Consequently, the use of flexible working hours and a home office is de-gendered, but there are power asymmetries connected to the levels of management and departments. Higher level managers seem to have more autonomy - and shoulder higher levels of stress and responsibilities, like Hobson and Fahlén (2009) assume in their research - while employees from certain departments, like customer service or property management, perceive they have limited access to these flexibility-enhancing opportunities. In addition, opportunities for flexibility are often overwritten by an inefficient meeting culture, extreme workloads and a lack of empowerment. Consequently, visibility and personal presence are required primarily due to the culture of meetings, even if the technology is available for online meetings. Employees do not perceive flexibility stigma in the sense that no one is marginalized for using a home office and flexible working hours, but the scope of usage might be limited due to the above-mentioned reasons.

Based on the agency and capabilities framework (Sen, 2008; Hobson et al., 2011), the sense of entitlement of men to claim a work-family balance is low if we take into consideration the fact that none of them took advantage of parental leave or parttime options to help satisfy family demands. Consequently, the agency of manager fathers does not truly challenge deeply held convictions about how work should be carried out, the cultural values of devotion to work, or the inevitability of unrestricted working hours (Lewis et al, 2007; Williams et al., 2013). Thus conversion factors, namely traditional gender norms (Takács, 2008, 2013; Pongrácz, 2001; Nagy, 2008), the deep-rooted ideal employee model (Acker, 2006) and the compulsion of visibility (Allard et al., 2007; Van Dyne et al., 2007; Halrynjo - Lyng, 2013) strongly determine the capabilities of Hungarian managers. Nonetheless, in the Hungarian context and compared to previous research (Nagy, 2008; Tóth, 2007; Primecz et al., 2014; Hobson et al., 2011) it is not negligible that manager fathers in the sample at least use some amount of flexibility to meet family demands, and that their use does not seriously impair their careers. Thus workplace organizational culture gives at least minimal room for manoeuvre in the form of home office and flexibility. This might be explained by the influence of having a Scandinavian leadership and parent company, a situation that was strongly praised by the interviewees due to its human-centeredness, informal ways 
of communicating and democratic values. Scandinavian societies are examples due to their policy legacy of promoting work-family balance (O'Brien et al, 2007) that might be influencing the corporate culture of subsidiaries, although in the Hungarian case exactly its gender equality values could not be transmitted. According to Utasi (2001), a democratic structure, like the Scandinavians employ, enhances the protection of employee rights, is less hierarchical, and generates a higher level of job security and trust. The inclusion of these aspects is especially crucial in the Hungarian working environment where the supervisor-subordinate relation is more hierarchical (Utasi, 2001), managerial control is closely intertwined with formal and informal work-family practices (Kispéter, 2012) and economic uncertainty is high (Hobson et al., 2011).

The situated agency of managers offers the greatest freedom for action, since most of them consider the organizational framework as a given and think only in terms of the opportunity to make individual changes. While men in previous research are not reported to want to change anything to create a better work-family balance (Nagy, 2008; Tóth, 2007), manager fathers from this sample had taken concrete steps to satisfy family demands. Just as Primecz (et al., 2014) concludes in her research, these activities remain at the individual level, and, according to Kvande (2009), as long as the take up of family-friendly policies remains an individual and optional choice, convictions about how work should be done will not be challenged. There are, however, those few but powerful leaders within the company who take into consideration their own influence on subordinates' work-family balance. These high-level managers make concrete changes regarding the norms of flexibility and try to mediate clear messages about expectations. This activity is very important since it offers guidelines in a boundless flexible environment and can act to alleviate the fear of employees by showing that it is acceptable to prioritise personal time demands over work (Hobson et al., 2011). Consequently, as many other researchers (Ladge et al., 2014; Rantanen et al., 2011; Primecz et al., 2014; Hobson et al., 2014; Allard et al., 2007; Holter, 2007) have already proven, informal relations are as important as formal work-life balance policies and managers have significant influence as role-models when it comes to mediating familyfriendly values. Consequently, until Hungarian workplace and family policies do not offer collective and standardised work-family provisions and forms of leave (such as, for example the father quota in Nordic societies), informal relations and good examples remain crucial for creating majority practice. 
The main limitation of this research comes from its use of a single case study, since findings can naturally not be generalized to the whole population. On the other hand, as an example it enriches existing empirical findings and contributes to the hitherto insufficient material about Hungarian men and work-life balance and might serve as a basis for future research as regards the formulation of hypotheses, defining theoretical concepts or making comparisons. Moreover, it draws attention to the complexity of the topic and show why use of a single work-life balance question in a quantitative survey might be found lacking or misleading, even if it produces representative results. Another limitation is that the interview method captures only discourse: what interviewees perceive, feel and think and what they are willing to share. Therefore, especially in the part about parental involvement, only information about how men evaluate their fathering contribution can be captured, while real behaviour and practice could be better measured using a time diary or shadowing. These methods, however, would have exceeded the capacity of this research effort. Moreover the research does not cover longitudinal data, therefore it is difficult to predict, whether perceiving balance at the moment means long lasting harmony or a temporary state of work-family balance. Given the often retrospective nature of interviews however, patterns and tendencies could be caught.

As for potential future research, it is important to expand the number of studies in which not only individuals but couples are interviewed, covering the crossover effect as well; namely, the transference of emotions and experiences to others such as family members. Although this dissertation was designed to include the partner dimension through the formulation of questions addressed to male managers, it is not the same as when a partner is directly interviewed. Another interesting topic for work-life balance research concerns the agency and capability options of individuals who are not allowed to use flexibility in harmonizing work and family life because of their positions or the nature of their jobs. Additionally, since there is a greater focus on flexibility, work-life balance provisions and white collar occupations, blue collar workers and employees in less privileged positions are often neglected in work-family research from the perspective of work autonomy. 


\section{REFERENCES}

Acker, J. (2006): Inequality Regimes. Gender, Class, and Race in Organizations. Gender \& Society, 20. (4): 441-464. DOI: 10.1177/0891243206289499

Acker, J. (2007): A „társadalmi nem és a szervezetek” irányzat jövője: kapcsolódások és határvonalak. In: Nagy B. (ed.): Szervezet, menedzsment és nemek. Budapest, Aula Kiadó: 13-30.

Acker, J. (2011): Theorizing Gender, Race, and Class in Organizations. In: Jeanes, E. L. - Knights, D. Martin, P. Y. (eds.): Handbook of Gender, Work \& Organization. West Sussex, Wiley.

Allard, K. - Haas, L. - Hwang, C.P. (2011): Family-Supportive Organizational Culture and Fathers' Experiences of Work-family Conflict in Sweden. Gender, Work and Organization, 18. (2): 141-157. DOI: $10.1111 /$ j.1468-0432.2010.00540.x

Allard, K. - Haas, L. - Hwang, P. (2007): Exploring the Paradox. Community, Work \& Family, 10. (4): 475-493. DOI: 10.1080/13668800701575135

Aycan, Z. - Eskin, M. (2005): Relative Contributions of Childcare, Spousal Support, and Organizational Support in Reducing Work-Family Conflict for Men and Women: The Case of Turkey. Sex Roles, 53. (7): 453-471. DOI: 10.1007/s11199-005-7134-8

Bell, A. S. - Rajendran, D. - Theiler, S. (2012): Job Stress, Wellbeing, Work-Life Balance and WorkLife Conflict Among Australian Academics. Electronic Journal of Applied Psychology. 8. (1): 25-37. DOI: http://dx.doi.org/10.7790/ejap.v8i1.320

Blaskó, Zs. (2006): Nők és férfiak - keresőmunka, házimunka. KSH NKI Kutatási jelentések 82. Budapest, KSH Népességtudományi Kutató Intézet

Bowman, D. (2007): Men's business. Negotiating entrepreneurial business and family life. Journal of Sociology, 43. (4): 385-400. DOI: 10.1177/1440783307083232

Brandth, B. - Kvande, E. (2001): Flexible Work and Flexible Fathers. Work Employment Society, 15. (2): 251-267. DOI: $10.1177 / 09500170122118940$

Broadbridge, A. - Hearn, J. (2008): Gender and Management: New Directions in Research and Continuing Patterns in Practice. British Journal of Management, 19: 38-49. DOI: 10.1111/j.14678551.2008.00570.x

Bryman, A. (1989): Research Methods and Organization Studies. London and New York, Routledge.

Burchell, B. - Fagan, C. - O’Brien, C. - Mark, S. (2007): Working conditions in the European Union: The gender perspective. Dublin, European Foundation for the Improvement of Living and Working Conditions.

Burchielli, R. - Bartram, T. - Thanacoody, R. (2008): Work-Family Balance or Greedy Organizations? Industrial Relations, 63. (1): 1-11. DOI : 10.7202/018124ar

Burke, R. (2000): Do managerial men benefit from organizational values supporting work-personal life balance? Women in Management Review, 15. (2): 81-89. DOI: 10.1108/09649420010319606

Burnett, S. - Gatrell, C. - Cooper C. - Sparrow, P. (2010): Fatherhood and flexible working: a contradiction in terms? In Kaiser, S. - Ringlstetter, M. J. - Pina Cunha, M. - Eikhof, D. R. (eds.): Creating balance? International perspectives on the work-life integration of professionals. Springer, Berlin. 157-171. 
Champoux, J. E. (1978): Perceptions of work and nonwork: A reexamination of the compensatory and spillover models. Sociology of Work and Occupations, 5. (4): 402-422. DOI: $10.1177 / 073088847800500402$

Chesley, N. (2011): Stay-at-Home Fathers and Breadwinning Mothers: Gender, Couple Dynamics, and Social Change. Gender \& Society, 25. (5): 642-664. DOI: 10.1177/0891243211417433

Cinamon, R. G. - Rich, Y. (2002): Gender Differences in the Importance of Work and Family Roles: Implications for Work-Family Conflict. Sex Roles, 47. (11-12): 531-541. DOI: 10.1023/A:1022021804846

Clark, S. C. (2000): Work/Family Border Theory: A New Theory of Work/Family Balance. Human Relations, 53. (6): 747-770. DOI: 10.1177/0018726700536001

Collinson, D. - Hearn, J. (1994): Naming Men as Men: Implications for Work, Organization and Management. Gender, Work and Organization, 1. (1): 2-22. DOI: 10.1111/j.1468-0432.1994.tb00002.x

Collinson, D. L. - Hearn, J. (1996): Breaking the Silence: On Men, Masculinities and Managements. In Collinson, D. L. - Hearn, J. (eds.): Men as Managers, Managers as Men. Critical Perspectives on Men, Masculinities and Managements. Sage Publications, London: 1-24. DOI: 10.4135/9781446280102.n1

Connell, R. W. - Messerschmidt, J. W. (2005): Hegemonic Masculinity: Rethinking the Concept. Gender \& Society, 19. (6): 829-859. DOI: 10.1177/0891243205278639

Connell, R. W. - Wood, J. (2005): Globalization and Business Masculinities. Men and Masculinities, 7. (4): 347-364. DOI: 10.1177/1097184X03260969

Connell, R. W. (2005): Globalization, Imperialism, and Masculinities. In: Kimmel, M. S. - Hearn, J. Connell, R. W. (eds.): Handbook of Studies on Men and Masculinities. Thousand Oaks, Sage Publications.

Connell, R. W. (2006a): Advancing gender reform in large-scale organizations: a new approach for practitioners and researchers. Policy and Society: journal of public, foreign and global policy, 24. (4): 121. DOI: $10.1016 / \mathrm{S} 1449-4035(05) 70066-7$

Connell, R. W. (2006b): Glass Ceilings or Gendered Institutions? Public Administration Review: 837849. DOI: $10.1111 / j .1540-6210.2006 .00652 . x$

Connell, R. W. (2009): Gender in World Perspective. Cambridge, Polity Press. DOI: $10.1080 / 14681811003666549$

Coser, L. A. (1974): Greedy Institutions. Patterns of Undivided Commitment. New York, The Free Press.

Critical Research on Men in Europe (CROME) (2005): Men, Masculinities, and „Europe”. In: Kimmel, M. S. - Hearn, J. - Connell, R. W. (eds.): Handbook of Studies on Men and Masculinities. Thousand Oaks, Sage Publications. DOI: 10.4135/9781452233833

Dén-Nagy, I. (2013): Az infokommunikációs technológiák munka-magánélet egyensúly megteremtésében játszott szerepe - elméleti áttekintés. Socio.hu, (3): 1-21. DOI: 10.18030/SOCIO.HU.2013.3.1

Dubin, R. (1973): Work and nonwork: Institutional perspectives. In: Dunnette, M. D. (ed.): Work and nonwork in the year 2001. Montgomery, Brooks/Cole: 53-68.

Duyvendak, J. W. - Stavenuiter, M. M. J. (2004): Working Fathers, Caring Men: Reconciliation of Working Life and Family Life. The Hague, Ministry of Social Affairs and Employment, Department for the Cordination of Emancipation Policy and Verwey-Jonker Institute.

Edwards, J. R. - Rothbard, N. P. (2005): Work and Family Stress and Well-being: An Integrative Model of Person-Environment Fit Within and Between the Work and Family Domains. In: Kossek, E. E. - 
Lambert, S. J. (eds.): Work and Life Integration. Organizational, Cultural, and Individual Perspectives. Mahwah, New Jersey, Lawrence Erlbaum Associates: 211-242.

Eek, F. - Axmon, A. (2013): Attitude and flexibility are the most important work place factors for working parents' mental wellbeing, stress, and work engagement. Scandinavian Journal of Public Health. 41.: 692-705. DOI: $10.1177 / 1403494813491167$

Emslie, C. - Hunt, K. (2009): 'Live to Work' or 'Work to Live'? A Qualitative Study of Gender and Work-life Balance among Men and Women in Mid-life. Gender, Work and Organization, 16. (1): 151172. DOI: $10.1111 / \mathrm{j} .1468-0432.2008 .00434 . \mathrm{x}$

Eräranta, K. - Moisander, J. (2011): Psychological Regimes of Truth and Father Identity: Challenges for Work/Life Integration. Organization Studies, 32. (4): 509-526. DOI: 10.1177/0170840611400293

Erin, A. C. - Blair-Loy, M. (2014): Consequences of Flexibility Stigma Among Academic Scientists and Engineers. Work and Occupations, 41. (1): 86-110. DOI: 10.1177/0730888413515497

Flyvbjerg, B. (2006): Five Misunderstandings About Case-Study Research. Qualitative Inquiry, 12. (2): 219-245. 10.1177/1077800405284363

Fodor, É. - Balogh, A. (2010): Back to the Kitchen: Gender Role Attitudes in 13 East European Countries. Journal of Family Research, 22. (3): 289-307.

Ford, J. - Collinson, D. (2011): In search of the perfect manager? Work-life balance and the managerial work. Work, employment and society, 25. (2): 257-273. DOI: 10.1177/0950017011398895

Forsberg, L. (2007): Negotiating involved fatherhood: Household work, childcare and spending time with children. Nordic Journal for Masculinity Studies, 2. (2): 109-126. DOI: 10.1177/0001699309104003

Frone, M. R. (2003): Work-family balance. In: Quick, J.C. - Tetrick, L. E. (eds): Handbook of occupational health psychology. Washington DC, American Psychological Association: 143-162.

Géring, Zs. (2014): Tartalomelemzés: A virtuális és a 'valós' világ határán. Egy vállalati honlap-elemzés bemutatása. Kultúra és Közösség, 4-5. (1): 9-24.

Geszler, N. (2014): A munka és a család konfliktusának forrásai az európai férfiak életében. Szociológiai Szemle, 24. (2): 65-89.

Greenhaus, J. H. - Beutell, N. J. (1985): Sources of conflict between work and family roles. Academy of Management Review, 10, (1): 76-88. DOI: 10.5465/AMR.1985.4277352

Greenhaus, J. H. - Collins, K. M. - Shaw, J. D. (2003): The relation between work-family balance and quality of life. Journal of Vocational Behavior, 63: 510-531. DOI: 10.1016/S0001-8791(02)00042-8

Greenhaus, J. H. - Parasuraman, S. (1999): Research on Work, Family, and Gender: Current Status and Future Directions. In: Powell, G. N. (ed.): Handbook of Gender \& Work. Thousand Oaks, Sage Publications: 391-413.

Greenhaus, J. H. - Powell, G. N. (2003): When work and family collide: Deciding between competing role demands. Organizational Behavior and Human Decision Processes, 90. (2): 291-303. DOI: 10.1016/S0749-5978(02)00519-8

Greenhaus, J. H. - Powell, G. N. (2006): When Work and Family are Allies: A Theory of Work-Family Enrichment. The Academy of Management Review, 31. (1): 72-92. DOI: 10.5465/AMR.2006.19379625

Gregory, A. - Milner, S. (2009): Work-life Balance: A Matter of Choice? Gender, Work and Organization, 16. (1): 1-13. DOI: 10.1111/j.1467-8543.2008.00710 
Grzywacz, J. G. - Carlson, D. S. (2007): Conceptualizing Work-Family Balance: implications for practice and research. Advances in Developing Human Resources, 9 (4): p. 455-471. DOI: $10.1177 / 1523422307305487$

Grzywacz, J. G. - Marks, N. F. (2000): Reconceptualizing the work-family interface: An ecological perspective on the correlates of positive and negative spillover between work and family. Journal of Occupational Health Psychology, 5. (1): 111-126. DOI: 10.1037/1076-8998.5.1.111

Guest, D. E. (2001): Perspectives on the Study of Work-Life Balance. Social Science Information, 41. (2): 255-279. DOI: 10.1177/0539018402041002005

Haas, L. - Rostgaard, T. (2011): Fathers' rights to paid parental leave in the Nordic countries: consequences for the gendered division of leave. Community, Work \& Family, 14. (2): 177-195. DOI: $10.1080 / 13668803.2011 .571398$

Hadas, M. (2011): A férfikutatások három hulláma. In: Hadas M. (ed.): Férfikutatások: TÁMOP onlineszöveggyüjtemény: 10-16.

Halrynjo, S. - Lyng, S. T. (2009): Preferences, constraints or schemas of devotion? Exploring Norwegian mothers' withdrawals from high-commitment careers. The British Journal of Sociology, 60. (2): 321-343. DOI: $10.1111 / \mathrm{j} .1468-4446.2009 .01233 . x$

Halrynjo, S. - Lyng, S. T. (2013): Fathers' parental leave in Norwegian Elite Professions. ESA Conference, Torino, 2013.08.30.

Halrynjo, S. (2009): Men's Work-life Conflict: Career, Care and Selfrealization: Patterns of Privileges and Dilemmas. Gender, Work and Organization, 16. (1): 98-125. DOI: 10.1111/j.14680432.2008.00432.x

Harcsa, I. (2014): Családi kohézió - A szülök és a gyermekek társas együttléte a mindennapok világában. A gyermekes családokban élők időfelhasználása. Budapest, KSH.

Hearn, J. (1992): Men in the Public Eye. Critical Studies on Men and Masculinities. London, Routledge. DOI: $10.1177 / 1097184 X 11432111$

Hill, E. J. - Yang, C. - Hawkins, A. J. - Ferris, M. (2004): A Cross-Cultural Test of the Work-Family Interface in 48 Countries. Journal of Marriage and Family, 66. (5): 1300-1316. DOI: 10.1111/j.00222445.2004.00094.x

Hobson, B. - Fahlén, S. - Takács, J. (2011): Agency and Capabilities to Achieve a Work-Life Balance: A Comparison of Sweden and Hungary. Social Politics, 18. (2): 168-198. DOI:10.1093/sp/jxr007

Hobson, B. - Fahlén, S. (2009): Competing Scenarios for European Fathers: Applying Sen's Capabilities and Agency Framework to Work--Family Balance. The ANNALS of the American Academy of Political and Social Science, 214-233. DOI: 10.1177/0002716209334435

Hochschild, A. - Machung, A. (1989): The Second Shift. New York, Penguin Books.

Hochschild, A. (2001): The Time Bind: When Work Becomes Home and Home Becomes Work. New York, Holt Paperbacks.

Hollway, W. (1996): Masters and Men in the Transition from Factory Hands to Sentimental Workers. In: Collinson, D. L. - Hearn, J. (eds.): Men as Managers, Managers as Men. Critical Perspectives on Men, Masculinities and Managements. Sage Publications, London: 25-42.

Holter, Ø. G. (2007): Men's Work and Family Reconciliation in Europe. Men and Masculinities, 9. (4): 425-456. DOI: 10.1177/1097184X06287794

Johansson, T. - Klinth, R. (2008): Caring Fathers: The Ideology of Gender Equality and Masculine Positions. Men and Masculinities, 11. (1): 42-62. DOI: 10.1177/1097184X06291899 
Kanter, R. M. (1993): Men and women of the corporation. New York, Basic Books.

Kelly, E. L. - Ammons, S. K. - Chermack, K. - Moen, P. (2010): Gendered Challenge, Gendered Response: Confronting the Ideal Worker Norm in a White-Collar Organization. Gender \& Society, 24. (3): 281-303. DOI: 10.1177/0891243210372073

Kerfoot, D. - Knights, D. (1996): 'The Best is Yet to Come?': The Quest for Embodiment in Managerial Work. In: Collinson, D. L. - Hearn, J. (eds.): Men as Managers, Managers as Men. Critical Perspectives on Men, Masculinities and Managements. Sage Publications, London: 78-98. DOI: $10.1177 / 135050840184003$

Kispéter, E. (2012): Producing Working Mothers: Maternalist Labor Control in a Hungarian Factory. Budapest, Central European University Department of Gender Studies.

Kitterød, R. H. - Rønsen, M. (2013): Does more involved fathering imply a double burden for fathers in Norway? Discussion Papers No. 753, Statistics Norway, Research Department

Korabik, K. - Lero, D. S. - Whitehead, D. L. (2008): Handbook of Work-Family Integration. Research, Theory and Best Practices. San Diego, CA, Elsevier.

Kossek, E. E. - Barber, A. E. - Winters, D. (1999): Using flexible schedules in the managerial world: The power of peers. Human Resource Management Journal, 38. (1): 36-46. DOI: 10.1002/(SICI)1099050X(199921)38:1<33::AID-HRM4>3.0.CO;2-H

Kossek, E. E. - Lautsch, B. A. (2012): Work-family boundary management styles in organizations. Organizational Psychology Review, 2. (2): 152-171. DOI: 10.1177/2041386611436264

Kossek, E. E. - Pichler, S. - Bodner, T. - Hammer, L. B. (2011): Workplace social support and workfamily conflict: a meta-analysis clarifying the influence of general and work-family-specific supervisor and organizational support. Personnel Psychology, 64. (2): 289-313. DOI: 10.1111/j.17446570.2011.01211.x

Kvande, E. (2009): Work-Life Balance for Fathers in Globalized Knowledge Work. Some Insights from the Norwegian Context. Gender, Work and Organization, 16. (1): 58-72. DOI: 10.1111/j.14680432.2008.00430.x

Ladge, J. - Humberd, B. - Harrington, B. - Watkins, M. (2014): Updating the Organization Man: An Examination of Involved Fathering in the Workplace. Academy of Management, Perspectives, 28. (4): 1 54. DOI: $10.5465 / \mathrm{amp} .2013 .0078$

Lamb, M. E., - Pleck, J. H., - Charnov, E. L., - Levine, J. A. (1987): A biosocial perspective on parental behavior and involvement. In: Lancaster, J. B. - Altman, J. - Rossi, A. (eds.): Parenting across the lifespan. New York, Academic Press: 11-42.

Lewis, S. - Gambles, R. - Rapaport, R. R. (2007): The constraints of a 'work-life balance' approach: an international perspective. The International Journal of Human Resource Management, 18. (3): 360-373. DOI: $10.1080 / 09585190601165577$

Martinengo, G. (2007): Gender differences and similarities in the work-family interface: The importance of considering family life stages. PhD dissertation. Downloaded on 11.12.2011 from the homepage of Harold B. Lee http://lib.byu.edu/cgibin/TxtOnly/betsie.pl/0001/contentdm.lib.byu.edu/ETD/image/etd2123.pdf

Maume, D. J. - Sebastian, R. A. - Bardo, A. R. (2010): Gender, Work-Family Responsibilities, and Sleep. Gender \& Society, 24. (6): 746-768. DOI: 10.1177/0891243210386949

Mayo, M. - Pastor, J. C. - Cooper, C. - Sanz-Vergel, I. A. (2014): Achieving work-family balance among Spanish managers and their spouses: a demands-control perspective. The International Journal of Human Resource Management, 22. (2): 331-350. DOI: 10.1080/09585192.2011.540158 
Mayring, P. (2014): Qualitative Content Analysis. Theoretical Foundation, Basic Procedures and Software Solution. Klagenfurt, 2014. Downloaded on 24.10.2015 from the website of SSOAR: http://nbnresolving.de/urn:nbn:de:0168-ssoar-395173

Mellner, C. - Aronsson, G. - Kecklund, G. (2014): Boundary Management Preferences, Boundary Control, and Work-Life Balance among Full-Time Employees Professional sin Knowledge-Intensive, Flexible Work. Nordic Journal of Working Life Studies, 4. (4): 7-23. DOI: http://dx.doi.org/10.19154/njwls.v4i4.4705

Morgan, D. (1996): The Gender of Bureaucracy. In: Collinson, D. L. - Hearn, J. (eds.): Men as Managers, Managers as Men. Critical Perspectives on Men, Masculinities and Managements. Sage Publications, London: 43-60. DOI: http://dx.doi.org/10.4135/9781446280102.n3

Nagy, B. (2007): A társadalmi nem szerepe a vezetésben Magyarországon - Érzékelése és magyarázatai. In: Nagy, B. (ed.) Szervezet, menedzsment és nemek. Budapest, Aula Kiadó: 110-120.

Nagy, B. (2008): A munka és a magánélet összeegyeztetése a férfi menedzserek életében. In: Balogh, P. Dobos, B. - Forgács, A. - Nagy, B. - Szúcs, A. (eds.): 60 éves a Közgazdaságtudományi Egyetem. A Jubileumi Tudományos Konferencia alkalmából készült tanulmányok. Társadalomtudományi Kar. Budapest, Aula Kiadó: 389-403.

Nagy, B. (2010): Obstacles and supports in women's career. In: Chybicka, A. - Safdar, S. F. Kwiatkowska, A. (eds): Culture and Gender: an Intimate Relation. Gdanks, GWP: 195-208.

Neff, L. A. - Karney, B. R. (2005): Gender Differeces in Social Support: A Question of Skill pr Responsiveness? Journal of Personality and Social Psychology, 88. (19): 79-90. DOI: 10.1037/00223514.88.1.79

Nordenmark, M. (2004): Balancing work and family demands: Do increasing demands increase strain? A longitudinal study. Scandinavian Journal of Public Health: 32.: 450-455. DOI: $10.1080 / 14034940410028280$

O’Brien, M. - Brandth, B. - Kvande, E. (2007): Fathers, Work and Family Life. Community, Work \& Family, 10. (4): 375-386. DOI: 10.1080/13668800701574971

OECD (2008): Babies and Bosses: Balancing Work and Family Life. Downloaded on 21.01.2012 from the homepage of OECD: http://www.oecd.org/dataoecd/12/2/34566853.pdf

Özbilgin, M. F. - Beauregard, T. A. - Tatli, A. - Bell, M. P. (2011): Work-life, diversity and intersectionality: a critical review and research agenda. International Journal of Management Reviews, 13. (2): 177-198. DOI: 10.1111/j.1468-2370.2010.00291.x

Plantin, L. (2007): Different classes, different fathers? Community, Work \& Family, 10. (1): 93-110. DOI: $10.1080 / 13668800601110835$

Pongrácz, T, - S. Molnár, E. (2011): Nemi szerepek és a közvélemény változásának kölcsönhatása. In: Nagy, I. - Pongrácz, T. (eds.): Szerepváltozások. Jelentés a nők és a férfiak helyzetéről 2011. Budapest, TÁRKI Nemzeti Erőforrás Minisztérium.

Pongrácz, T. (2001): A család és a munka szerepe a nők életében. In: Nagy, I. - Pongrácz, T. - Tóth, I. Gy. (eds): Szerepváltozások: Jelentés a nők és férfiak helyzetéről 2001. Budapest, TÁRKI.

Primecz, H. - Kiss, Cs. - Toarniczky, A. - Csillag, S. - Szilas, R. - Bácsi, K. - Milassin, A. (2014): Magyarországi „munkavállaló-barát” (employee friendly) szervezetek - Valóság vagy utópia (avagy mit tanulhatunk tőlük?). $\quad$ Vezetéstudomány, $45 . \quad 40)$ (10): $2-16$.

Rantanen, J. - Kinnunen, U. - Mauno, S. - Tillemann, K. (2011): Introducing Theoretical Approaches to Work-Life Balance and Testing a New Typology Among Professionals. In: Kaiser, S. - Ringsltetter, M. J. 
- Eikhof, D. R. - Pina e Cunha, M. (eds.): Creating Balance? International Perspectives on the Work-Life Integration of Professionals. Berlin, Springer. DOI 10.1007/978-3-642-16199-5_2

Rantanen, J. (2008): Work-Family Interface and Psychological Well-Being: A Personality and Longitudinal Perspective. PhD dissertation. Downloaded on 11.12.2011 from the website of Jyväskylä University: https://jyx.jyu.fi/dspace/bitstream/handle/123456789/19200/9789513934255.pdf?sequence=1

Rehel, E. M. (2013): When Dad Stays Home Too: Paternity Leave, Gender, and Parenting. Gender \& Society, 28. (1):110-132. DOI: 10.1177/0891243213503900

Roehling, P. - Moen, P. - Batt, R. (2003): Spillover. In: Moen, P. (ed.): It's about time: Couples and careers. Ithaca NY, Cornell University Press: 101-121

Rothbard, N. P. - Dumas, T. L. (2004): Research perspectives: managing the work-home interface. In: Jones, F. - Burke, R. J. - Westman, M. (eds.): Work-Life Balance. A Psychological Perspective. Hove and New York, Psychology Press: 71-89.

Rudberg, M. - Nielsen, H. B. (2012): The making of a 'new man': Psychosocial change in a generational context. Journal of Psycho-Social Studies, 6. (1): 55-74.

Saxonberg, S. - Sirovatka, T. (2006): Failing Family Policy in Post-Communist Central Europe. Journal of Comparative Policy Analysis, 8. (2): 185 - 202. DOI: 10.1080/13876980600682089

Sebők, Cs. (2015): A női munkavállalás hatása a napi időfelhasználásra. Doktori (PhD) értekezés, Budapesti Corvinus Egyetem, Szociológia Doktori Iskola. DOI 10.14267/phd.2015028

Sen, A. (2008): Capability and Well-Being. In: Hausman, Daniel M. (ed.): The Philosophy of Economics. An Anthology. Third Edition. Cambridge, Cambridge University Press: 270-293.

Sieber, S. D. (1974): Toward a Theory of Role Accumulation. American Sociological Review, 39. (4): 567-578. DOI: $10.2307 / 2094422$

Spéder, Zs. (2011): Ellentmondó elvárások között... Családi férfiszerepek, apaképek a mai Magyarországon. In. Nagy, I. - Pongrácz, T. (eds.): Szerepváltozások: Jelentés a nők és férfiak helyzetéröl 2011. Budapest, TÁRKI Nemzeti Erőforrás Minisztérium.

Szalma, I. - Takács, J. (2013): Should men have more rights...? Gender-role-related attitudes before and during the 2008 crisis. In: Jónsson, G. - Stefánsson, K. (eds.): Retrenchment and Renewal? Welfare States in Times of Economic Crises (Studies in Historical Welfare State Research 6.). Helsinki, Nordic Centre of Excellence NordWel: 207-234.

Szalma, I. (2014): Hogyan alakult a kétkeresős családok munka és magánélet közötti konfliktusa a válság hatására 2004 és 2010 között Európában? Szociológiai Szemle, 24. (1): 102-129.

Takács, J. (2008): Ha mosogatógép nem lenne, már elváltunk volna. Férfiak és nők otthoni munkamegosztása európai összehasonlításban. Esély, 19. (6):51-73.

Takács, J. (2013): Unattainable desires? Childbearing capabilities in early $21^{\text {st }}$ century Hungary. In: Oláh, L. Sz. - Fratczak, E. (eds.): Childbearing, Women's Employment and Work-Life Balance Policies in Contemporary Europe. Basingstoke and New York, Palgrave Macmillan: 179-206.

Takács, J. (2015): Aktívan törődő apák Magyarországon. “A bizonytalanság szociológiája” MSZT konferencia, Budapest, November 20.

Tammelin, M. (2009): Working Time and Family Time. Experiences of the Work and Family Interface among Dual-Earning Couples in Finland. PhD dissertation. Downloaded on 11.12.2011. from the homepage of University of Tampere: http://www.uta.fi/laitokset/sostut/tutkimus/labournet/pdf/Tammelin_dissertation.pdf 
Tausig, M. - Fenwick, R. (2001): Unbinding Time: Alternate Work Schedules and Work-Life Balance. Journal of Family and Economic Issues, 22. (2): 101-119. DOI: 10.1023/A:1016626028720

Thompson, A. (2010): Men, masculinities and flexible working. International Industrial Relations Association Conference, Copenhagen University, Denmark.

Tóth, H. (2007): „Struggle for Life”: Munka és magánélet egyensúlya dilemmái a nemek közötti különbségek tükrében. In: Nagy, B. (ed.): Szervezet, menedzsment és nemek. Budapest, Aula Kiadó.

Utasi, Á. (2011): Szubjektív feszültség és munkastressz a házasok életében. Összehasonlítás Európa 24 országában. In. Nagy, I. - Pongrácz, T. (eds.): Szerepváltozások: Jelentés a nők és férfiak helyzetéről 2011. Budapest, TÁRKI Nemzeti Erőforrás Minisztérium.

Van der Lippe, T. - Jager, A. - Kops, Y. (2006): Combination pressure. The paid work-family balance of men and women in European countries. Acta Sociologica, 49. (3): 303-319. DOI: $10.1177 / 0001699306067711$

Van Dyne, L. - Kossek, E. E. - Lobel S. (2007): Less need to be there: Cross level effects of work practices that support work-life flexibility and enhance group processes and group-level ocb. Human Relations, 60. (8): 1123-1153. DOI: 10.1177/0018726707081657

Van Echtelt, P. - Glebbeek, A. - Lewis, S. - Lindenberg, S. (2009): Post-fordist work: a man's world? Gender and Working Overtime in the Netherlands. Gender \& Society, 23. (2):188-214. DOI: $10.1177 / 0891243208331320$

Voydanoff, P. (2002): Linkages between the work-family interface and work, family, and individual outcomes: An integrative model. Journal of Family Issues, 23. (1): 138-164. DOI: $10.1177 / 0192513 \times 02023001007$

Wajcman, J. - Bittman, M. - Brown, J. E. (2008): Families Without Borders: Mobile Phones, Connectedness and Work-Home Divisions. Sociology, $43 . \quad$ (4):635-652. DOI: $10.1177 / 0038038508091620$

Wajcman, J. - Edwards, P. (2005): The Politics of Working Life. New York, Oxford University Press

Wall, G. - Arnold, S. (2007): How Involved is Involved Fathering?: An Exploration of the Contemporary Culture of Fatherhood. Gender \& Society, 21. (4): 508-527. DOI: 10.1177/0891243207304973

Williams, J. C. - Blair-Loy, M. - Berdahl, J. L. (2013): Cultural Schemas, Social Class, and the Flexibility Stigma. Journal of Social Issues, 69. (2): 209-234. DOI: 10.1111/josi.12012

Yin, R. K. (2003). Case Study Research. Design and Methods. Thousand Oaks, Sage Publications. 


\section{OWN PUBLICATIONS REGARDING THE TOPIC}

\section{Publications in Hungarian}

\section{Refereed Journal Articles}

Geszler, Nikolett [2014]: Sources of Work-Family Conflict of European Men. Szociológia Szemle, 24. (2): 65-89

Geszler, Nikolett [2014]: Work-Life Balance of Manager Men. Kultúra és Közösség, 5. (4): $115-122$.

\section{Book chapters}

Geszler, Nikolett - Ördög, Zita [2011]: Income of Leaders - Main Findings of an International Research. In: Wimmer Ágnes - Novák Zsuzsanna - Bartus Tamás - Jenes Barbara (eds.): Közgáz Diáktudós. Business Models, Economic and Social Effects. Selection from the Works of Scientific Students' Associations. Budapest, Alinea Kiadó: $127-142$.

\section{Conference Presentation}

Geszler, Nikolett [2013]: Manager Men. Budapest, Joint Jubilee Conference of Hungarian Sociological Association and the Hungarian Academy of Sciences Institute for Sociology.

\section{Publications in English}

\section{Refereed Journal Articles}

Geszler, Nikolett [in press]: Behaviour-based Conflict in the Life of Hungarian Manager Fathers. Intersections, East European Journal of Society and Politics, Special Issue „Work-life balance/imbalance: individual, organizational and social experiences”

\section{Book chapters}

Lengyel, György - Geszler, Nikolett - Ördög, Zita [2014]: Income and Influence. Hungarian, Polish and German business leaders compared. In: Bluhm, Katharina Martens, Bernd - Trappmann, Vera (eds.): Business Leaders and New Varieties of Capitalism in Post-Communist Europe. Routledge, London: 205-219.

\section{Conference Presentation}

Geszler, Nikolett [2016]: Time-based Work-Family conflict in the Life of Hungarian Manager Fathers. Łódź, 1st Internetional Conference „Women and Men Facing Everyday Challenges. Work and Life Demands in the Context of Gender"

Geszler, Nikolett [2015]: Work-Family Conflict of Hungarian Male Managers: TimeBased Conflict. Prague, 12th Conference of the European Sociological Association. 


\section{APPENDIX}

\section{Interview Guide}

1. To what extent do you feel that your work and private life are in balance? What do you understand by work-life balance?

2. Is there anything you would change to achieve a better work-life balance? (If yes): what are those and what prevents you from realization?

3. What are the sources of conflicting situations? (time, strain, behaviour)

4. Who do you receive support from to achieve work-life balance?

5. How does your partner relate to your career? Were there any situations or periods when she was not as supportive as you expected? And the children?

6. How do you support your partner?

7. Have you ever felt a sense of guilt towards your family (you have not dedicate enough time or attention to them; you have missed important family events, etc.)? And towards your work?

8. What strategies do you apply to decrease the tensions, which arise from work and family demands? (asking about paid service: babysitter, cleaner; information technology)

9. To what extent can you or want you to separate work from private life in everyday life?

10. Have you ever needed to sacrifice something to maintain work-life balance (for example a career abroad, leisure time, studying, friends)?

11. In which life domain you feel the most successful or the most confident?

12. What changes fatherhood has brought to your life? What where the best parts and what the challenges?

13. According to you what are the most important tasks of a father?

14 . What do you enjoy the most in your managerial work?

15. How compatible are flexible provisions with managerial career? How can fatherhood and the managerial work be harmonized?

16. Do you know about any provision the company provides for employees to achieve a better work-life balance? Who are these provisions primarily targeting? Which are the ones you are also benefiting from? 